\title{
Multi-criteria decision-making for coronavirus disease 2019 applications: a theoretical analysis review
}

\author{
M. A. Alsalem ${ }^{1}$ - A. H. Alamoodi ${ }^{1}$ - O. S. Albahri ${ }^{1}$ (D) K. A. Dawood ${ }^{2}$ 'R. T. Mohammed ${ }^{2}$. \\ Alhamzah Alnoor ${ }^{3}$ - A. A. Zaidan ${ }^{4}$ - A. S. Albahri ${ }^{5}$ B. B. Zaidan ${ }^{6}$ - F. M. Jumaah ${ }^{7,8}$. \\ Jameel R. Al-Obaidi ${ }^{9}$
}

Published online: 27 January 2022

(c) The Author(s), under exclusive licence to Springer Nature B.V. 2022

\begin{abstract}
The influence of the ongoing COVID-19 pandemic that is being felt in all spheres of our lives and has a remarkable effect on global health care delivery occurs amongst the ongoing global health crisis of patients and the required services. From the time of the first detection of infection amongst the public, researchers investigated various applications in the fight against the COVID-19 outbreak and outlined the crucial roles of different research areas in this unprecedented battle. In the context of existing studies in the literature surrounding COVID-19, related to medical treatment decisions, the dimensions of context addressed in previous multidisciplinary studies reveal the lack of appropriate decision mechanisms during the COVID-19 outbreak. Multiple criteria decision making (MCDM) has been applied widely in our daily lives in various ways with numerous successful stories to help analyse complex decisions and provide an accurate decision process. The rise of MCDM in combating COVID-19 from a theoretical perspective view needs further investigation to meet the important characteristic points that match integrating MCDM and COVID-19. To this end, a comprehensive review and an analysis of these multidisciplinary fields, carried out by different MCDM theories concerning COVID19 in complex case studies, are provided. Research directions on exploring the potentials of MCDM and enhancing its capabilities and power through two directions (i.e. development and evaluation) in COVID-19 are thoroughly discussed. In addition, Bibliometrics has been analysed, visualization and interpretation based on the evaluation and development category using R-tool involves; annual scientific production, country scientific production, Wordcloud, factor analysis in bibliographic, and country collaboration map. Furthermore, 8 characteristic points that go through the analysis based on new tables of information are highlighted and discussed to cover several important facts and percentages associated with standardising the evaluation criteria, MCDM theory in ranking alternatives and weighting criteria, operators used with the MCDM methods, normalisation types for the data used, MCDM theory contexts, selected experts ways, validation scheme for effective MCDM theory and the challenges of MCDM theory used in COVID-19 studies. Accordingly, a recommended MCDM theory solution is presented through three distinct phases as a future direction in COVID19 studies. Key phases of this methodology include the Fuzzy Delphi method for unifying criteria and establishing importance level, Fuzzy weighted Zero Inconsistency
\end{abstract}

Extended author information available on the last page of the article 
for weighting to mitigate the shortcomings of the previous weighting techniques and the MCDM approach by the name Fuzzy Decision by Opinion Score method for prioritising alternatives and providing a unique ranking solution. This study will provide MCDM researchers and the wider community an overview of the current status of MCDM evaluation and development methods and motivate researchers in harnessing MCDM potentials in tackling an accurate decision for different fields against COVID-19.

Keywords COVID-19 · Multicriteria decision making · Evaluation · Development · Characteristics

\section{Abbreviations}

AHP

AHP-DEMATEL AHP-VIKOR

ARAS

BWM

BWM- TODIM

BWM-TOPSIS

COPRAS

CQROULWPBM

DEMATEL

F-AHP

F-DEMATEL-F-ANP

FFNs

FLQOWA

Fuzzy RAFSI

GRA

HFS-ARAS

IFSs

IMEEM

ImFT

ISM

LBWA-MACBETH-RAFSI

LHFSs

MARCOS

MCDM

MULTIMOORA
Analytical hierarchy process

AHP-decision making trial and evaluation laboratory

AHP-VlseKriterijuska Optimizacija I Komoromisno

Resenje

Additive ratio assessment

Best-worst method

BWM-acronym in Portuguese for interative multi-criteria decision making

BWM-technique for order of preference by similarity to ideal solution

Complex proportional assessment

Complex Q-rung orthopair uncertain linguistic weighted partitioned Bonferroni mean

Decision-making trial and evaluation laboratory

Fuzzy-AHP

Fuzzy-DEMATEL-fuzzy-analytic network process

Fermatean fuzzy sets

Fuzzy linguistic quantifier order weighted aggregation

Fuzzy ranking of alternatives through functional mapping of criterion sub-intervals into a single interval

Grey relational analysis

Hesitant fuzzy set-ARAS

Intuitionistic fuzzy set

Improved matter-element extension model

Intuitionistic m-polar fuzzy topology

Interpretive structural modeling

Level-based weight assessment-measuring attractiveness by a categorical-based evaluation technique-ranking of alternatives through functional mapping of criterion subintervals into a single interval

Linguistic hesitant fuzzy sets

Measurement of alternatives and ranking according to compromise solution

Multi criteria decision making

Multi-objective optimization on the basis of ratio analysis plus full multiplicative form multiobjective optimization by ratio analysis 
NTS

PAPRIKA

PFDOSM

PFN

PFWZIC

PLP

PROMETHEE

q-ROFDOSM

q-ROFWZIC

SFN

SFSs

SODOSM

SpNoFWBM

SWARA

TFNs

TODIM

TrIT2F

T-SFDOSM

UPLZs

WASPAS
Neutrosophic time series

Potentially all pairwise rankings of all possible alternative

Pythagorean fuzzy decision by opinion score method

Pythagorean fuzzy set

Pythagorean fuzzy-weighted zero-inconsistency

Piecewise linear prospect

Preference ranking organization method for enrichment evaluation

Q-rung orthopair fuzzy decision by opinion score method Q-rung orthopair fuzzy-weighted zero-inconsistency

Scheme fuzzy logic and network

Spherical fuzzy sets

Subjective and objective decision by opinion score method Spherical normal fuzzy weighted Bonferroni mean

Stepwise weight assessment ratio analysis

Triangular fuzzy number

Acronym in Portuguese for interative multi-criteria decision making

Trapezoidal interval type-2 fuzzy

T-spherical fuzzy sets-fuzzy decision by opinion score method

Linguistic Z-number with unbalanced semantics

Weighted additive sum product assessment

\section{Introduction}

From the beginning of the coronavirus (COVID-19) pandemic, researchers have been responding rapidly, working on different disciplines around the world to unify efforts and provide their contributions to stop this disease. As COVID-19 overwhelmed healthcare systems around the world, having a knock-on effect on the diagnosis and treatment of other challenges, fears are increasing about the effect of the pandemic on people's health and the future consequences of this disease (Cousins 2020). The World Health Organization (WHO) has emphasised that one of the most important things to address regarding the COVID-19 pandemic is to understand and evaluate the risk factors for this disease (Rod et al. 2020; Moreira and da Costa 2020). A global agreement is that COVID-19 has changed the direction of different fields of studies such as medical-, social-, economic-, technologies-, service- and patient-related fields (He and Harris 2020; Zeng et al. 2020), and an essential need exists for understanding how different cases and perspectives for these fields can be evaluated. Moreover, the global COVID-19 pandemic has had a considerable effect on the scientific enterprise, including scholarly publications concerning different COVID-19 cases focusing on different factors and/or criteria analyses (Myers et al. 2020; Demirjian et al. 2019). Thus, using the information derived from the analysis is of paramount importance to support efficient, rapid operational response/decisions for our life decision support systems because scientific publications addressing the pandemic are being produced rapidly (Dagens et al. 2020; Boin and 't Hart 2010).

Artificial Intelligence (AI), an emerging technology in the field of medicine, has contributed actively to fight COVID-19 through different theories (Chaudhari et al. 2020). In 
this worldwide health crisis, the medical industry is looking for how AI technologies can detect, evaluate and control the spread of COVID 19 or the infected cases to assess this outbreak from different viewpoints (Xu et al. 2020). In particular, the utilisation of existing decision-making (DM) algorithms is widely accepted and confers different contributions and implications. Capacity evaluation of different contributions for COVID-19 using multicriteria decision-analysis (MCDA) techniques has been widely used to reveal the relationships amongst the evaluation criteria and select different alternative cases (Ward et al. 1557). In addition, the development of a new family of DM algorithms for pandemic COVID-19 adaptation and learning is specifically tailored to decision COVID-19 issues and problems, and constructed by building upon principles from decision theory. Multicriteria decision-making (MCDM) is considered a complex DM tool involving quantitative and qualitative factors, and recently, several MCDM techniques and approaches have been suggested for evaluating multiple criteria and selecting the optimal probable amongst alternatives (Mardani et al. 2000). In these contexts, the integration analysis between COVID19 and MCDM that might help answer three sequential questions are presented in this introduction, in which the matter question (first question) can be set as below:

\subsection{How can DM techniques assist in alleviating COVID-19?}

MCDM is an upcoming, useful tool for evaluating different COVID-19 criteria and helps in selecting the best choice; thus, it can provide different benefits to eliminate COVID-19 conditions or cases as well as the infected patients concerning any DM process (Albahri et al. 2020a). Moreover, it can substantially improve treatment consistency and DM by developing useful algorithms (Ghorui et al. 2021). MCDM is helpful in controlling COVID19 infected patients, providing comprehensive analyses for criteria used and affording the precise standardised and guideline for the number and type of criteria (Mohammed et al. 2021a). It can track COVID-19 at different scales such as medical, molecular and epidemiological applications (Albahri et al. 2020b). It is also helpful for facilitating the research on this virus using analysis of the available data (Abdel-Basst et al. 2020a). Additionally, MCDM can help in developing proper decision regimens, prevention strategies, drug and vaccine development with an exact judgment (Albahri et al. 2021a). The global COVID-19 pandemic has led to major challenges in clinical (i.e. when the demand for intensive care exceeds local capacity) and nonclinical DM. To promote consistent, transparent, objective and ethical DM, decision theory formed a solution to develop urgently guidelines outlining key principles that should be utilised during the pandemic. Accordingly, another important question that needs to be answered is as follows:

\subsection{How has MCDM contributed to the COVID-19 pandemic?}

The academic literature touches on two essential directions of MCDM methods in the context of COVID-19 case studies: evaluation and development. The evaluations of different case studies by using several MCDM methods concerning multiple criteria have been utilised to rank the performance of alternatives and widely applied as the first direction (Albahri et al. 2021b). Ongoing progress in the academic literature has contributed to improving the DM mechanism through MCDM methods such as developing a framework that provides accurate services, handling perfect detection, conducting seamless treatment or facilitating vaccine distribution in the context of COVID-19 as the second direction (Albahri et al. 2021a). However, more investigation analyses and aspects need 
to be conducted when integration occurs between MCDM and COVID-19 for evaluation and development categories. To acquire an optimal decision mechanism analysis based on MCDM theory, the use of an overarching concept enables discussing COVID-19 perspectives from various MCDM theoretical parts focusing on comprehensive analysis for the COVID-19 studies in evaluation and development directions. Taken together, these directions should highlight and discuss the fact that different characteristics of COVID-19 in different case studies might be associated with standardising the evaluation criteria (Mohammed et al. 2021a), MCDM theory in ranking alternatives and weighting criteria (Albahri et al. 2021b; Salman et al. 2017), the operators used with the MCDM methods (Hamid et al. 2021), the normalisation types for the used data (Albahri et al. 2020), MCDM theory contexts, selected experts ways (Albahri et al. 2020d), validation scheme for effective MCDM theory (Sheares 2020) and challenges of MCDM theory used in COVID-19 studies (Thomas 2020).

\subsection{What are the research novelty and contributions?}

To the best of the authors' knowledge, no research attempted to review the use of MCDM in COVID-19 applications comprehensively. Thus, the presented study aims to analyse the MADM techniques intensively and demonstrate all the current work directions in the context of COVID-19 to provide an exhaustive analysis for researchers. The contributions of this study are as follows:

1. A comprehensive review and analysis base are presented to classify academic literature into two categories, namely, evaluation and development in the context of COVID-19 for the first time.

2. Standardised steps in the evaluation of COVID-19 criteria and ranking alternatives based on MCDM theory are reported.

3. Different COVID-19 cases under MCDM theory operators and normalisation technique are discussed.

4. Multiple aggregation methods are highlighted for multiexpert MCDM methods and analysed in the context of COVID-19 perspectives.

5. Several validation schema parameters of MCDM methods are investigated and discussed in terms of appropriateness and suitability for COVID-19 cases.

6. Theoretical challenges related to COVID-19 case studies are addressed on the basis of weighting and ranking MCDM methods to provide the current potential future directions and key solutions.

7. Future research directions for COVID-19 with MCDM with three distinct phases are presented.

Importantly, a comprehensive view of all presented sections and methodology is offered because it comprises the ability to view this study with a complete viewpoint, as visualised in Fig. 1. 


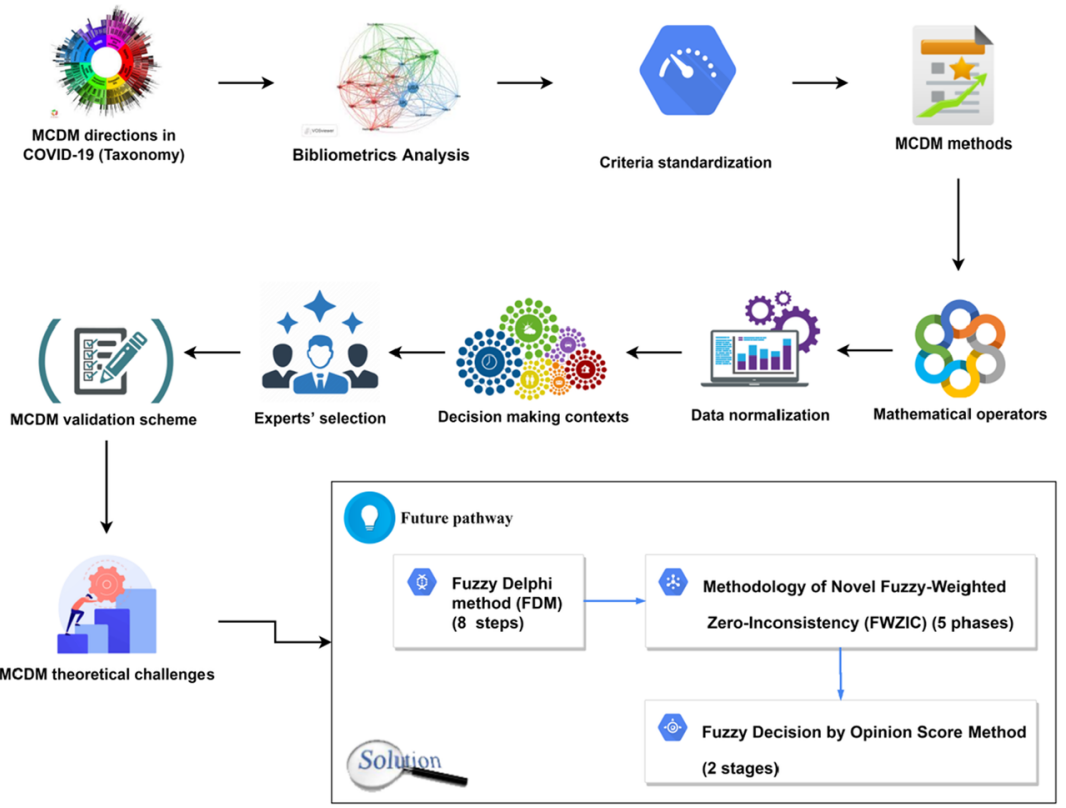

Fig. 1 Flow of moverall MCDM analysis in the context of COVID-19

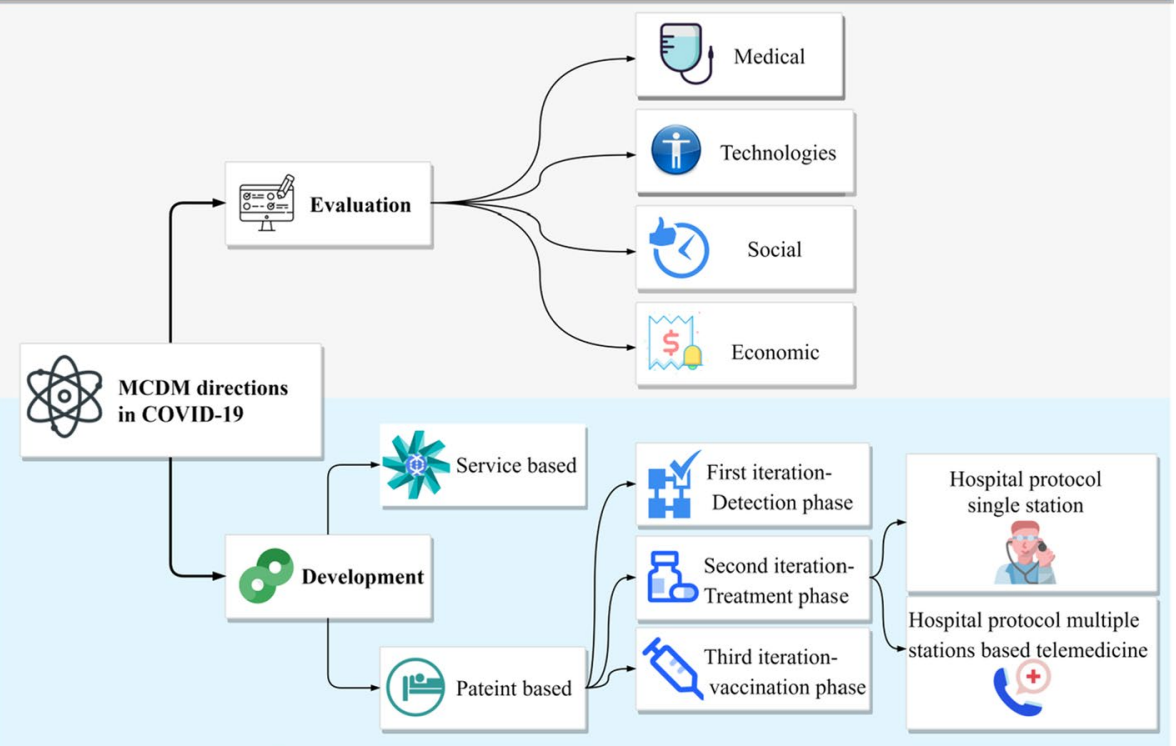

Fig. 2 Taxonomy of the DM works in the context COVID-19 


\section{Comprehensive taxonomy and results}

This study explores and analyses the literature of DM problems in the context of COVID19. A total of 55 studies considered such issue and dealt with it from two perspectives, namely, evaluation and development based, as demonstrated in the taxonomy diagram in Fig. 2.

Noticeably, (41,74.5\%) of articles focused on evaluation and were categorised under 'evaluation-based' category that uses the MCDM approach for evaluating different related cases in the context of COVID-19. Accordingly, four subcategories are driven based on the evaluated cases, namely, medical-, social-, economic- and technologies-related.

However, $(14,25.45 \%)$ of the studies were categorised in terms of 'development-based,' which refers to related works that utilised MCDM methods in developing a framework that provides services, detection and treatment, or facilitates vaccine distribution in the context of COVID-19. This category is composed of two subcategories based on development direction, namely, service- and patient-based. The research works under the service-based subcategory attempted to develop or propose new service such as (health system policy service (Requia et al. 2019), COVID-19 online-diagnosis service (Ahmad et al. 2021) or even provide better Internet service to handle the increasing user demand during COVID19 that caused to control movement (Abdulsalam and Hossain 2020)). The patient-based subcategory is divided into three iterations of research work efforts in dealing with the COVID-19 pandemic, starting with the first iteration of the COVID-19 detection, followed by treatment iteration and ending with the third iteration of the vaccine distribution mechanism.

\subsection{Evaluation}

The 'evaluation-based' category comprises four subcategories mentioned in Fig. 2, namely, medical-, social-, economic- and technologies-related. Table 1 illustrates the percentages of frequency distributions for these four subcategories that utilise MCDM in the context of COVID-19.

Table 1 presents the percentage of distribution studies for each of the four subcategories under the evaluation-based perspective. The medical cases dominated more than half (51\%) of the research studies, followed by the economic-related cases (32\%), less than that for social- and technology-related cases with (10\% and 7\%), respectively.

In the medical subcategory, out of all studies in evaluation-based category, 21 research works focused on evaluating medical-related cases that cover three major evaluation issues, namely, strategies and policies, medical tools and medical facilities. In first study (Pamučar et al. 2020), an MCDM method was used to develop long-term strategic guidelines for reorganising a healthcare system in the aftermath of the COVID-19 epidemic. The authors argued that the MCDM approach would help in emergency scenarios like the present pandemic, which made the integration of the technique important for the establishment of sufficient medical treatment for patients and the reduction of risks to the public caused by the COVID-19 pandemic to the minimum. In their investigation, the researchers employed four strategic guidelines that were established along with five assessment criteria. In the following study by Samanıloğlu and Kaya 2020, the authors examined the hospitals' confrontation with a wide range of possibilities represented in preparing COVID-19 preventative measures. They did not attempt to determine which alternatives were superior, but they utilised MCDM to identify these alternatives, organise 
Table 1 Distribution frequencies of evaluation category

\begin{tabular}{lllll}
\hline Ref of study & Medical & Social & Economic & Technologies
\end{tabular}

Pamučar et al. (2020)

Samanıloğlu and Kaya (2020)

Ocampo and Yamagishi (2020)

Sarwar and Imran (2021)

Wu and Xu (2020)

Yang et al. (2020)

Mahanta and Panda (2020)

Sayan et al. (2020)

Mohammed et al (2020)

Abdel-Basst et al. (2020b)

Albahri et al. (2020)

Garg et al. (2020)

Yang et al. (2021)

Shirazi et al. (2020)

Naeem et al. (2020)

Ortiz-Barrios et al. (2020)

Wan et al. (2021)

Manupati et al. (2021)

Zhang et al. (2020)

Bharsakade et al. (2021)

Mishra et al. (2019)

Baz et al. (2020)

Ashraf and Abdullah (2020)

Jain et al. (2021)

Ghorui (2021)

Grida et al. (2020)

Jamshidiantehrani et al. (2020)

Sharma et al. (2020)

Khurana et al. (2020)

Ghosh et al. (2020)

Moslem et al. (2020)

Shah et al. (2020)

Lam et al. (2021)

Duan et al. (2021)

Yao (2021)

Altuntas and Gok (2021)

Ecer and Pamucar (2021)

Althaf and Babbitt (2021)

Chauhan et al. (2020)

Mardani et al. (2020)

Gong et al. (2021)

Percentages \% 
them according to their relevance and compare various preventative methods amongst countries worldwide. The study by Ocampo and Yamagishi (2020) addressed a considerable issue raised with the presence of COVID-19. The authors asserted that the mental and physical wellbeing of persons subjected to lockdowns has emerged as a growing worry. From one side, the governments are constantly attempting to pursue lockdown relaxation efforts to maintain public health and to recommence economic activity. On another side, they have endeavoured to retain exit strategies whilst avoiding further waves of cases. Such a major issue would be deemed a contradictory criterion, especially when the government relies on a trial-and-error strategy. When the relaxation strategy is needed and the guideline protocols for relaxation strategies are interconnected, MCDM emerges to provide a better solution.

In another study by Sarwar and Imran (2021), the authors addressed how numerous health organisations, including the WHO and many governmental agencies, were striving to provide suitable recommendations and appropriate measures to minimise the spread of COVID-19. In their quest, the implementation of all suggestions by people is impractical because of the many social and physical measures such as social and physical distances, use of antiviral masks, prevention of needless travel, maintenance of hygiene, healthy food consumption and health monitoring. As a result, strategies that are more effective in reducing the spread of COVID-19 should be prioritised above with less influence. Consequently, an MCDM analysis was performed to analyse and prioritise COVID-19 preventative measures. In the following study by $\mathrm{Wu}$ and $\mathrm{Xu}$ (2020), the authors claimed that because of the necessity of emergency medical services during the COVID-19 pandemic, their final, harmonious integration is required. They emphasised that the response to the epidemic influences the rate of alleviating its issues. They also discussed how to respond effectively to the urban pandemic situation in a complicated context, which has become a worldwide concern. Another important consideration in the emergency setting is that emergency DM is typically an MCDM problem, which involves several criteria or features with qualitative and quantitative elements. As a result, the severity of the urban COVID-19 epidemic condition within the context of a complex humanitarian catastrophe can be assessed using MCDM. In the study by Yang et al. (2020), the mask selection issue was raised during the COVID-19 pandemic. The authors suggested that the selection of antivirus masks is serious because of the scarcity of such instruments during the pandemic. People frequently believe that the best masks are either pricey or used solely by frontliners. This misunderstanding leads to the improper, excessive procurement and use of personal protective equipment, exacerbating mask scarcity. In actuality, masks should be evaluated with aspects such as reusability, raw material quality and the situations of persons to optimise their use and that of medical resources. When such a problem arises, MCDM contributes to the selection of an acceptable antiviral mask based on the demands and situations of many people, thus guaranteeing practical importance. Similarly, the authors in Mahanta and Panda (2020) stipulated that in light of the COVID-19 epidemic, selecting the appropriate face mask has become a challenge for many people due to the absence of specific standards. MCDM was used to solve this problem. The authors in Sayan et al. (2020) asserted that precisely and rapidly diagnosing patients is crucial in their treatment. Moreover, the availability of multiple diagnostic tests causes uncertainty in determining the most appropriate procedure. Prioritising one above the other is a challenging decision, especially when several factors are considered, such as high sensitivity, high specificity, low false positivity, low false negativity, good usability and low cost. As a result, the authors used the MCDM approach to evaluate the effectiveness of seven different COVID-19 diagnostic tests. They found that the efficiency of diagnostic testing varies depending on the person's situation and the resources available in each country. 
In Mohammed et al. (2020), the research highlighted the wide use of the AI model in diagnosing COVID-19. These AI techniques are particularly desirable in assisting healthcare organisations in selecting a proper COVID-19 diagnosis system. However, as with any other technology, they are confusing and have disadvantages that limit their ultimate application. The authors claimed that selecting one technique over the other is not an easy task due to the availability of numerous machine learning (ML) models, and the evaluation and benchmarking of COVID-19 ML in such situation is the main concern, especially when multiple criteria are involved. Thus, adapting the MCDM method can solve such problem. Based on the study by Abdel-Basst et al. (2020b), diagnosing COVID-19 via $\mathrm{CT}$ is challenging and ambiguous because the symptoms associated with it resemble other viral lung infections, such as H1N1, H5N1, SARS and hantavirus. Accordingly, the authors identified such problems as complex DM problems that involve multiple conflict criteria. They employed the MCDM approach in the context of an uncertain environment to assess numerous viral lung disease types including COVID-19 as alternative crossover with symptoms and results of CT imaging as evaluation criteria. The authors believed their work can help medical professionals monitor the expansion of COVID-19 by providing reliable evaluation results. Another engaging work (Albahri et al. 2020) considered the possibility of employing MCDM to detect COVID-19. Three phases were established for the COVID-19 AI classification methods to perform the diagnostics of the disease. Researchers started with the identification phase of the preprocessing and the datasets, then defined the criteria used to evaluate and benchmark COVID-19 AI classification algorithms as well as the number and type of techniques. As the result of this phase, four decision matrices were produced. Next, the integration of the MCDM approached was applied to solve the issue. Finally, the validity of the output was examined objectively and subjectively for further verification. Another diagnosis evaluation study (Garg et al. 2020) presented an approach for dealing with MCDM challenges based on operators. To prove the applicability of their proposed technique, the authors used COVID-19 as a case study for selecting an appropriate laboratory test. The study by Yang et al. (2021) followed, where the authors explored the importance of mask selection during the COVID-19 epidemic. The six most common and available masks were assessed using MCDM methods. They also examined the benefits and drawbacks as well as conducted a comparison study with graphical interpretation.

The authors in Shirazi et al. (2020) studied the dissatisfaction of the patient towards hospital services in the presence of COVID-19. Despite resources and medical capacities, frustration with inappropriate allocation prevents patients from experiencing the ultimate health experience in terms of their wants and wishes. They suggested that prioritising patient service aspects sustains service quality. Consequently, MCDM was introduced to solve the decision problem. To prosper and survive in today's competitive environment, the technique assisted in identifying which satisfaction aspects should be prioritised under normal conditions and during the COVID-19 pandemic. Another study by Naeem et al. (2020) discussed several methods to minimise COVID-19 exposure, including treatment approaches. The authors employed a new concept of the MCDM method to explore the best treatment alternative for COVID-19, based on their suggested theory of the applied case. The next study with respect to evaluation to hospitals and their resources by Ortiz-Barrios et al. (2020) highlighted the necessity of hospital readiness, as it is the primary platform for those seeking healthcare services during disaster time. Moreover, many uncertainties and decision concerns arise that make recognising what these hospitals can actually offer difficult. Thus, identifying hospitals that are unprepared is critical for disaster planning. The authors claimed that the COVID-19 epidemic is currently straining the health sector. In such cases, hospital preparation should be swift and reliable. As a result, MCDM was used 
to assess hospital disaster preparedness and was found to be an appropriate technique given the dearth of methodologies for assessing hospital disaster preparedness levels. Another study by Wan et al. (2021) pointed out the importance of hospital selection in the aftermath of COVID-19. When COVID-19 broke out in Wuhan, China, the authors focused on the hospital selection challenge. Accordingly, the government highlighted the importance of 'leave no one unattended'. The Chinese government ordered the design of temporary hospitals in Wuhan to support the health system. Undoubtedly, this initiative created substantial DM challenges regarding hospital selection to provide efficient treatment for patients with moderate symptoms of COVID-19. Consequently, MCDM was utilised to select the best solution from many possible alternatives. Additionally, resource works addressed several issues, including the disposal of medical waste. The authors in Manupati et al. (2021) described how 'many authorities, particularly in poor countries, are striving to select the optimal health care waste (HCW) disposal technology for the effective treatment of medical wastes during and after the COVID-19 era. Selecting the optimum technique necessitates considering many concrete and intangible characteristics, which can be presented as a complex DM problem. Hence, they devised a framework for HCW disposal selection that incorporates socio-technical and triple bottom-line considerations.

In another treatment evaluation study by Zhang et al. (2020), the authors stated that whilst treating patients with COVID-19 with possible medications is required, people with COVID-19 infection would experience fever, cough, shortness of breath, dyspnoea and other symptoms. COVID-19-associated disorders are difficult to treat because no specific medication is available. In that context, MCDM was employed to select the medicine for COVID-19. Another work by Bharsakade et al. (2021) discussed the necessity of recognising the varied characteristics of seven major forms of waste in healthcare procedures. MCDM was utilised for prioritising the identified wastes and their dimensions. The authors also established a framework for evaluating and validating resilience in the healthcare system. In Mishra et al. (2019), the authors claimed that the selection of the proper antiviral treatment to treat mild symptoms of COVID-19 is complex, challenging and unknown because no definitive treatment exists at the time and other treatment options emerged including numerous antiviral medications. The authors used hesitant fuzzy sets (HFSs) to select five techniques or medicines to cure moderate symptoms of COVID-19 and illustrate feasibility and effectiveness of the proposed idea in real life.

In the social subcategory, four research works focused on evaluating the social aspect with the context of COVID-19. In the first work by Baz et al. (2020), the COVID-19 effect on several critical centres across the world must be analysed to determine the socioeconomic issues that countries face. Amongst these social dimensions, the most critical matter is still unidentified. MCDM has been employed to arrange these many factors in a logical order and help with the associated difficulty. To combat COVID-19, multiple preventive actions have been classified. Numerous variances between the cases have been noted, and the appropriate procedure has not yet been determined. Therefore, MCDM was utilised to determine the optimal preventive technique. The next study by Ashraf and Abdullah (2020) indicated that given the increasing catastrophe factors during the COVID-19 pandemic, several organisations have had DM issues for their emergency operations during these times. To deal with these issues, the authors employed the MCDM technique to explore DM under ambiguity, which can help them in emergency DM issues. The next work (Jain et al. 2021) found that employers who mainly depend on handheld device (HHD) during their daily work have a higher risk of musculoskeletal problems. The authors stated that due to COVID-19, working from home increased the number of hours spent in inconvenient postures. This problem caused musculoskeletal diseases (MSDs) in HHD users. 
The authors presented an MCDM strategy for assessing MSD risk amongst HHD users. Another work by Ghorui (2021) discussed COVID-19 risk factors. The COVID-19 pandemic in December 2019 affected the whole world. The outbreak is spreading quickly in many ways because the virus is highly infectious. The authors explored sorting risk factors and ranking them in terms of spread as a way to control the infection using MCDM methods.

In the economic subcategory, 13 studies dealt with four main topics, namely, supply chain, green economy, transportation and environment. According to the work presented in Grida et al. (2020), the authors introduced a deep-dive analysis of the ramifications of COVID-19's disruption as well as its economic consequences. They contended that the COVID-19 supply chain issue is caused by three distinct factors: (1) supply, (2) demand and (3) logistics. Several studies have examined the influence of preventive initiatives on supply chain policy. MCDM was utilised to create sufficient information for industrial enterprises' decision makers to solve uncertainty issues, which are classified as highdecision issues connected with the three previously described supply chain policies. The next study (Jamshidiantehrani et al. 2020) investigated the challenges that hampered pharmaceutical businesses' supply chain agility during the COVID-19 crisis, particularly, the delayed financial turnover and the necessity for immediate financial resources. MCDM was used to improve prediction accuracy, cut production costs, optimise resource utilisation and establish criteria for selecting the best suppliers to maximise production speed and flexibility.

Next, the authors in Sharma et al. (2020) demonstrated how the COVID-19 epidemic has affected supplier businesses' ability to construct systems that are more responsive and resilient to future industrial crises. MCDM was utilised to discover the most essential aspects that aid in the rebuilding of enterprises and communities such that they can better withstand crises and be more adaptable. The authors in Khurana et al. (2020) highlighted various unforeseen hazards as a result of agricultural supply chain disruptions during the COVID-19 pandemic. Another considerable problem is that the substantial threats throughout this worldwide crisis remain unknown. Agricultural supply chain risks are prioritised using MCDM methodologies, including demand risks, financial risks, logistical and infrastructure risks, management and operational risks, policy and regulatory risks, and biological and environmental hazards. In the next work by Ghosh et al. (2020), the authors evaluated the changes in ambient variables caused by COVID-19 lockdowns. They had different alternatives in their study. They properly studied indicators by using an MCDM method. Moreover, they learned about the effect of the COVID-19 pandemic lockdown situation on the environment and the assessment of the situation after the lockdown. In Moslem et al. (2020), the authors argued that countries suffered as a result of the transportation sector's sustainability and faced several environmental issues due to the difficulty in identifying transportation alternatives. This issue arose after adopting COVID-19 social distancing measures. MCDM was used to find transportation choices that would improve the urban surroundings. Aside from the supply chain, another topic for green economy was discussed by authors in Shah et al. (2020). They suggested that the COVID-19 situation is an opportunity to change towards a green economy because of the curfew concerns that have affected numerous companies, creating a favourable opportunity to cut carbon production. Despite this, businesses have a difficult time deciding on which kind of waste-to-energy conversion to prioritise. Thus, an MCDM with fuzzy priorities for waste to energy conversion was created to alleviate uncertainty. Another green energy work was discussed by Yao (2021). The authors argued that COVID-19 posed a major challenge to global health and global development regime. Environmental rules and policies for green growth and 
development are one of the primary difficulties faced by specialists, economists, politicians and governments. To solve this issue, the authors used MCDM to analyse environmental regulations. They identified alternate strategies whilst considering several environmental regulations main and sub criteria.

The authors in Lam et al. (2021) highlighted the effect of COVID-19 on economic development for the countries involved, including their respective governmental agencies and the construction industry, urging the industry to build an advanced infrastructure designed to enhance health, transport, education and housing. The MCDM approach was implemented because no complete study was undertaken on the evaluation of construction company financial performance. The authors used the offered approaches to determine the importance of financial ratios and the ranking of construction enterprises. In Duan et al. (2021), the authors discussed the influence of COVID-19 on electric power grid investment. They highlighted how, as a result of COVID-19, China's socioeconomic development is changing, and that it is critical to assess the risk of electric power grid investment in China in light of the current socioeconomic development environment, which may assist investors manage risk and prevent risk loss. The MCDM method was presented to assist in assessing the risks of investing in China's electric power grid as a result of changed socioeconomic and regulatory conditions. In Altuntas and Gok (2021), the authors explored another critical application of MCDM in light of a void in the scientific literature on making the appropriate quarantine decisions to mitigate the detrimental effect of a pandemic on the hospitality sector. The authors suggested that during a pandemic, quarantine choices should be made systematically, and no general protocol exists, notably during the COVID-19 epidemic. The authors used the DEMATEL technique to help governments lessen the detrimental effect of quarantine decisions on the hospitality business owing to the COVID-19 epidemic. In the next work (Ecer and Pamucar 2021), the authors examined how COVID-19 has affected the medical sector with COVID-19-related decisions and challenges, which have also been expressed in business and the economy. They reviewed the insurance industry and how rating and ranking private health insurance providers might help agencies, customers and authorities make better insurance decisions. An MCDM ranked insurance companies based on their healthcare services given in Turkey during the COVID-19 outbreak. In conclusion, the new approach used during the COVID-19 pandemic managed to overcome the insurance assessment challenge. Another supply chain disruption work by Althaf and Babbitt (2021) emphasised the importance of electronic devices in society, but practically all human direct communication has become virtual (i.e. online) due to COVID-19. As a result of the worldwide health issues and their effect on climate change, the supply of crucial raw materials for electronic manufacture is becoming increasingly vulnerable. A multicriteria framework employing TOPSIS was used to assess supply chain disruption risks in the electronics sector.

In the technologies subcategory, three research works focused on evaluation of technology facet with the context of COVID-19. In the first work by Chauhan et al. (2020), the authors discussed how waste is a considerable harm to the environment and public health. Especially in light of the COVID-19 outbreak, effective waste disposal has become a critical risk to human life. Thus, one issue is determining the criteria for a smart system for industrial medical waste disposal. By utilising MCDM to ascertain the link between healthcare facilities and waste management firms, the researchers reduced and handled the challenges in the most efficient manner feasible. The second work by Mardani et al. (2020) discussed how digital technologies are now regarded as one of the most important components of daily existence. Although COVID-19 has recently emerged, it has also introduced multiple problems due to its various strengths, limitations, possibilities and threats. 
MCDM was used to assess the importance of these aspects in connection to digital technology in the health industry. The study found that the most remarkable variable affecting health information systems is health knowledge systems, which is followed by the absence of digital knowledge, digital stratification and economic interventions. Another technological issue is the use of Internet resources and technologies in academic contexts. Lastly, the authors in Gong et al. (2021) described how 'under the influence of the COVID-19 pandemic, network teaching has been widely established to ensure the implementation of teaching plans and defend students' learning rights'. They also highlighted the existence of numerous online resources in this regard, and how selecting a certain website for online teaching can have a direct effect on educator and student performance as well as promote the quality of online teaching. As a result, e-learning website selection can be viewed as a complex MCDM problem, and expert assessments of e-learning website performance are frequently imprecise and hazy due to the subjectivity inherent in human thinking.

\subsection{Development}

The category of 'development based' consists of two subcategories, namely, service-based and patient-faced, as presented in Fig. 2. However, the developing new framework in patient-faced passed through three main iterations to handle the COVID 19 pandemic. The first iteration is Detection Phase, where the research efforts focus on developing a decision support system based on MCDM for detecting the spread of the epidemic or the infected cases, followed by the second iteration, Treatment Phase, where the research efforts pay more attention to developing an MCDM framework for providing traditional treatment and handling the patient either in single or multiple stations. Lastly, the third iteration is Vaccine Phase, where MCDM is used to develop a new framework to handle vaccine distribution and prioritisation issue. Table 2 illustrates the percentages of frequency distributions of these two subcategories that utilise MCDM in the context of COVID-19.

Table 2 presents the percentage of distribution studies for each of the four subcategories under development-based perspective. Out of 14 studies $(5,35.71 \%)$ were for the servicebased, whereas the patent-based dominated more than half of the development research with $(9,64.28 \%)$ which was decomposed in three iterations: detection phase with $(2,14 \%)$, treatment phase (hospital protocol single station with $(2,14 \%)$ and hospital protocol multiple stations-based telemedicine with $(1,7 \%))$ and vaccination phase with $(4,29 \%)$.

In the service-based subcategory, the research efforts worked developing and providing service in the context of COVID-19. In the first work by Requia et al. (2019), arguments were towards finding a solution for minimising the COVID-19 effect imposed on healthcare systems. A new concept was introduced where MCDM was used to address the issues of bed capacity for COVID-19 positive cases and provide an effective support in terms of hospitalisation policies for national, regional and local intervention. The study by Ashraf et al. (2020) was also meant to support the emergency control for COVID-19. Authors were concerned with using MCDM for controlling the transmission and spread of COVID-19 owing to the difficulty of deciding during such times; thus, MCDM was a better solution to address that gap. In the study (Ahmad et al. 2021), the authors discussed the importance of addressing COVID-19 issues, especially those related to treatment. Fuzzy Cloud-Based COVID-19 Diagnosis Assistant was proposed. This system's main responsibility is to distinguish patients as confirmed, suspected or probable COVID-19 cases, and categorise those infected as mild, moderate, severe or critical. The tool presented excellent feedback and performance, especially with respect to monitoring the COVID-19 pandemic 


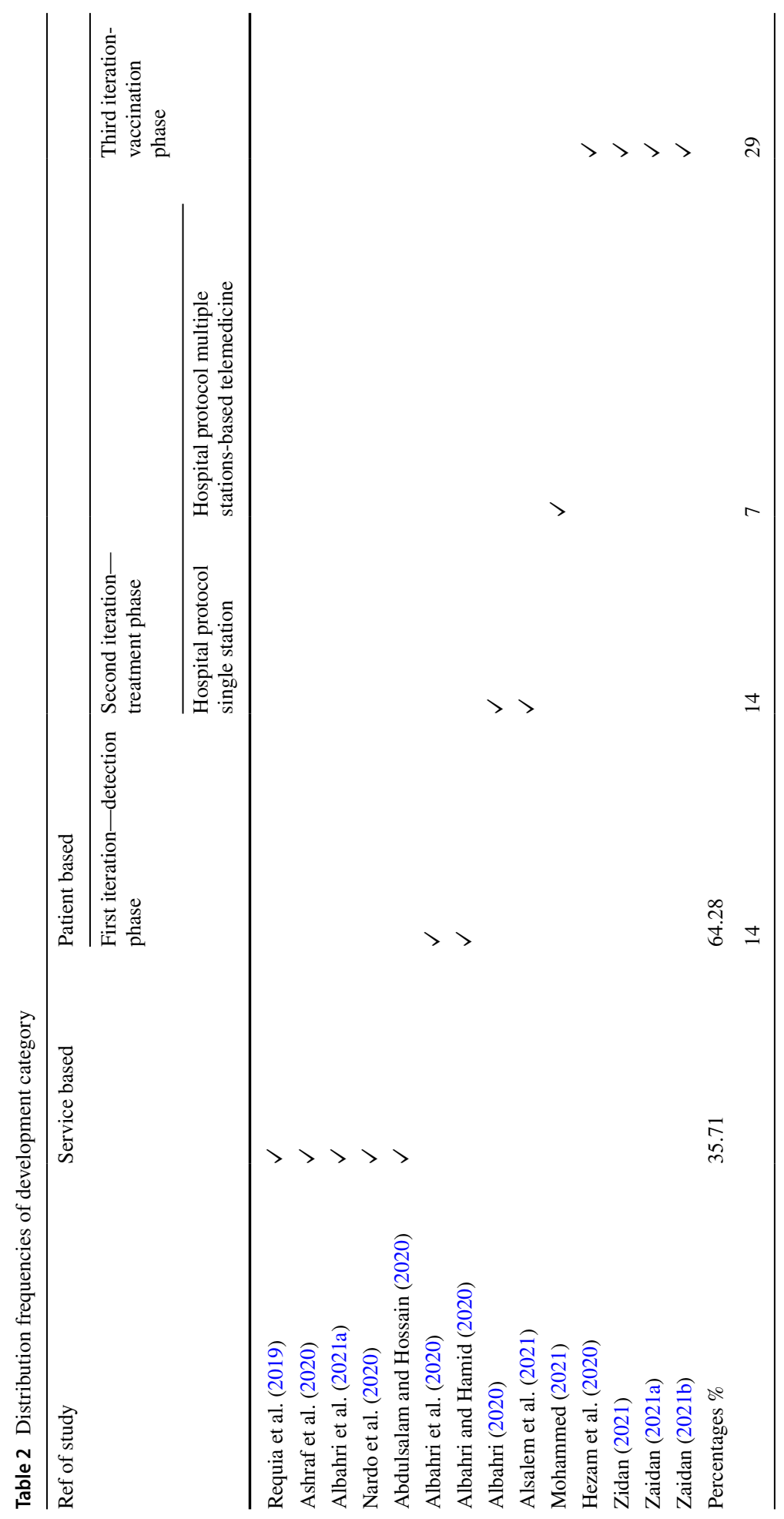


and reducing its transmission rate amongst the public. The fourth set discussing MCDM with services included 14 studies. The first three studies discussed patients. In Nardo et al. (2020), the hospital bed shortage admission issue during the COVID-19 pandemic was presented. Such issue not only raised the concern for existing patients but also made the medical institutions handicapped with their limited resources from receiving extra patients. This issue was particularly apparent in low- and middle-income settings. MCDM was utilised for prioritising access to care in these situations where noncritical patients with COVID-19 were identified and others in more serious conditions were admitted to hospitals because of the risk of rapid clinical deterioration, which assisted the response of health systems. Lastly, in Abdulsalam and Hossain (2020), the authors discussed the telecommunication services and their challenges in meeting customer demands during the COVID-19 pandemic. Such issue was not only owing to the increased customer demand but also because most of the employees were working from their homes, which were not as equipped as their offices to address customer needs.

The patient-based subcategory in return comprised three iterations for handling COVID-19 issue. The first two studies focused on COVID-19 detection iteration. In Albahri et al. (2020), the authors in the academic literature discussed the increasing cases of patients with COVID-19, and medical institutions are faced with many issues in making tough decisions owing to such increase. To address such issue, medical centres rely on using MCDM to prioritise patients by considering the urgency for a solution to minimise risks for deteriorating conditions. This solution is meant to mitigate current challenges and complications of patients with severe COVID-19 based on laboratory examination criteria used to differentiate between serious and critical COVID-19 conditions. In the second study (Albahri and Hamid 2020), the authors discussed the spread of the COVID-19 along with the need to address its issues from medical perspectives. Authors raised the need for a novel design solution, especially for asymptomatic patients due to multilaboratory criteria, criterion importance and trade-off amongst these criteria, which are challenging to differentiate except when MCDM concept is applied. MCDM was used for patient prioritisation and detecting their health conditions of asymptomatic carriers.

The three following studies were under the treatment iteration, and the first two discussed the development of hospital protocol for single station. In Albahri (2020), patients who recently recovered from COVID-19 have antibodies against the virus circulating in their blood, and such antibodies can be used to boost the immune system of patients in deteriorating conditions. The issue encountered in that research was to whom these antibodies should be given first in the presence of biologically different and conflicted criteria. Therefore, a rescue framework was suggested for the transfusion of the best convalescent plasma (CP) to the most critical patients whilst considering using ML and MCDM. In the second work (Alsalem et al. 2021), the authors discussed utilising MCDM to address the issue of mesenchymal stem cells. They proposed a transfusion framework based on MCDM methods. The issues in which MCDM was proposed addressed not only including efficient transfusion to the most critical cases of COVID-19 but also differentiating patients into different emergency levels automatically and prioritising them in each emergency level. Their work provided prevention and health promotion of patients infected with COVID19. The third study dealt with the development of hospital protocol for multiple stationsbased telemedicine; in Mohammed (2021), the authors discussed the spread of COVID-19 across the world, raising the need for $\mathrm{CP}$ transfusion to the most critical patients that can assist in preventing the spread of COVID-19. In their strategy, a novel CP-transfusion intelligent framework for rescuing patients with COVID-19 across centralised/decentralised 
telemedicine hospitals. The system main goal was to provide $\mathrm{CP}$ efficiently from eligible donors to the most critical patients using MCDM methods.

The most recent and engaging iteration is the third one, namely, Vaccination Phase, where three studies worked on development of a vaccine distribution mechanism. In the first study in Hezam et al. (2020), the COVID-19 vaccine was discussed. The main highlight presented the government need to set and identify priority groups for allocating COVID-19 vaccine doses. The authors discussed an MCDM strategy to assist in classifying and ranking the most deserving groups in society to receive the vaccine first. Followed by Zidan (2021), the authors discussed the issue of vaccine distribution for different groups of people. Their main idea was utilising MCDM for prioritising vaccine recipients and proposing a framework for distributing the COVID-19 vaccine. They utilised an artificial record of 300 recipients based on different COVID-19 vaccine distribution criteria. Their findings suggested the applicability of their method in addressing such issue of vaccine distribution and its need worldwide. In the study by Zaidan (2021a), the same authors of the previous manuscript discussed utilising MCDM for prioritising vaccine recipients with a more robust, stronger MCDM approach. They used a new fuzzy environment to address the uncertainty issues found in many MCDM context problems, especially those that can be linked with vaccine distribution for COVID-19. The last vaccine study was also proposed by the same group of authors in Zaidan (2021b), where the issue of vaccine distribution was addressed using a new formulation of MCDM approach. Their main ideas were using an integration of fuzzy-weighted zero-inconsistency and fuzzy decision by opinion score methods (FDOSMs) under a q-rung orthopair environment for COVID-19 vaccine dose distribution. Their findings not only suggested the powerfulness of their proposed method but also its effect and effectiveness.

\subsection{Bibliometric analysis}

The number of academic studies on MCDM and COVID-19 is increasing rapidly (Albahri et al. 2021a). Consequently, keeping up with such studies that are published is impossible. The focus on empirical contributions has led to massive streams of research (Hezam et al. 2021). Hence, the increasing studies reduce the sufficiency of gathered evidence and knowledge based on previous studies (Aria and Cuccurullo 2017). In this context, scholars adopt different approaches to review earlier studies to understand and reorganise the findings (Rodríguez-Soler et al. 2020). Bibliometrics can provide a transparent review on the basis of the statistical measurement of scientific activity (Aria and Cuccurullo 2017). Bibliometrics has become helpful because it allows for tremendous, valuable information for academics and practitioners in various disciplines. However, bibliometric analysis considers several phases that utilise numerous and mapping software tools (Io and Lee 2017). This study adopted a comprehensive science mapping analysis based on the R-tool for comprehensive bibliometric analyses. These tools provide a set of statistical and graphic techniques for classifying and reorganising knowledge. Thus, adopting such a tool is justified because it is helpful in a continuously evolving domain. Moreover, the bibliometric tool is helpful for mapping analysis, including data analysis, data visualisation and interpretation based on the evaluation and development category (Aria and Cuccurullo 2017), as described in the following sections. 
Fig. 3 Several included articles in different categories by year of publication

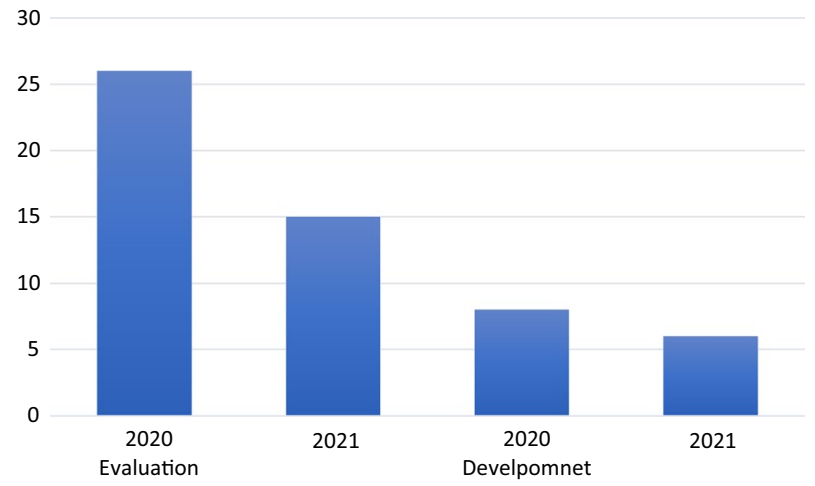

\subsubsection{Annual scientific production}

Figure 3 indicates the number of articles included in the two categories according to the year of publication and the distribution of scholarly papers from 2020 to 2021 .

The analysis shows the most research articles were produced in 2020 for the evaluation category and 2021 for the development category. A substantial portion is in the evaluation category because of the recent increase in issues connected with this matter. Additionally, an increase has been observed in the development category. Using MCDM methods with COVID-19 is vital to solving complex problems. Hence, the literature for this field of science has increased in recent years. For the evaluation category, increases of $63 \%$ and $37 \%$ were observed for 2020 and 2021, respectively. However, for the development category, increases of 57\% and 43\% were noted for 2020 and 2021, respectively. Moreover, this growth is due to the MCDM approach providing solutions to many problems related to COVID-19, such as industrial, medical and commercial.

\subsubsection{Country scientific production}

Figure 4 shows the 23 countries and nationalities represented the evaluation category on MCDM and COVID-19, whilst the 12 countries and nationalities came from the development category.

Figure 4 shows the most exhilarating countries producing MCDM and COVID-19 topics are India, China and Turkey because they are the countries that have suffered most during COVID-19 issues. Hence, they would look for more decisive decisions based on the evaluation category for MCDM methods to solve medical, social and economic problems. However, many countries have adopted MCDM methods to solve COVID-19 problems related to patients and services based on the development category. The most exciting countries producing MCDM and COVID-19 topics for the development category are Malaysia and Iraq because they are developed countries. According to the country scientific production, the highest percentage is for India with $73 \%$ because it has a weak health system that has exacerbated the threat of COVID-19 to society. Therefore, emphasis was placed on the evaluation category in MCDM methods to find scientific solutions. However, for the development category, the highest percentage is for Malaysia with $78 \%$ because it ranks second worldwide in the worsening of COVID-19 problems relative to its population. 


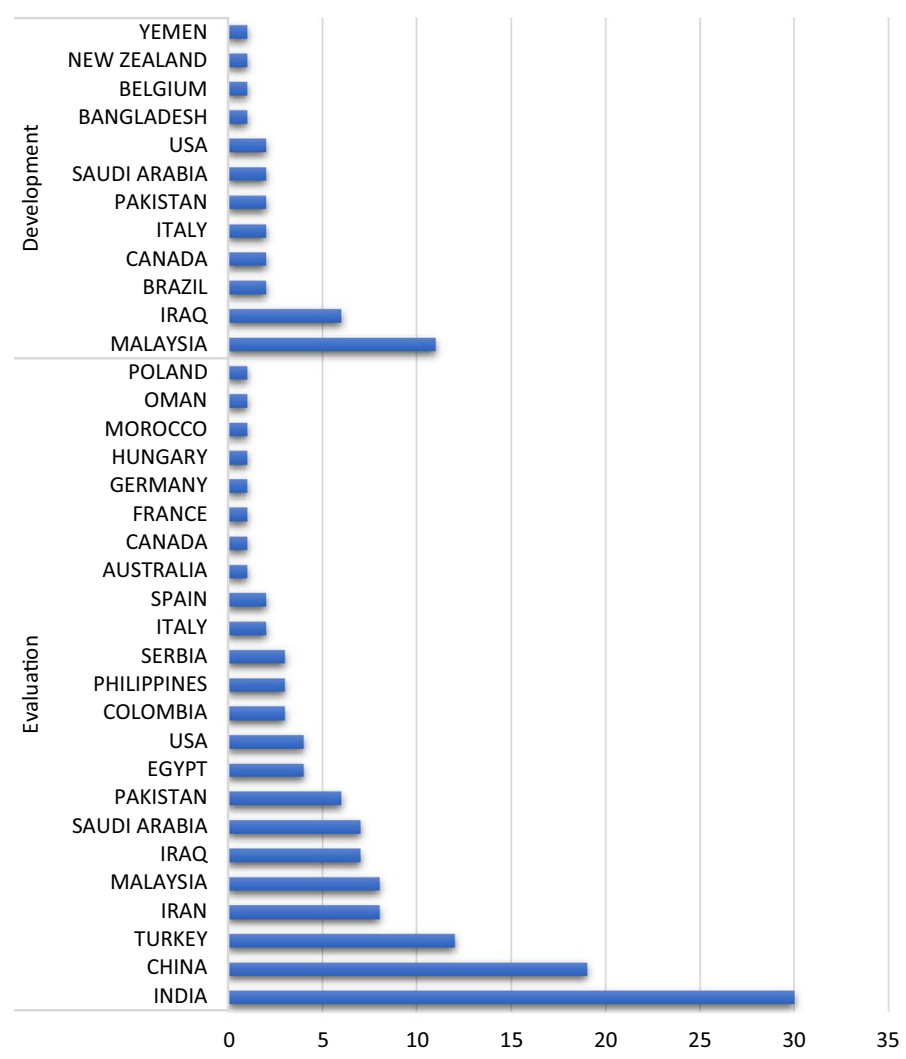

Fig. 4 Country scientific production

\section{WordCloud based on development and evaluation category}

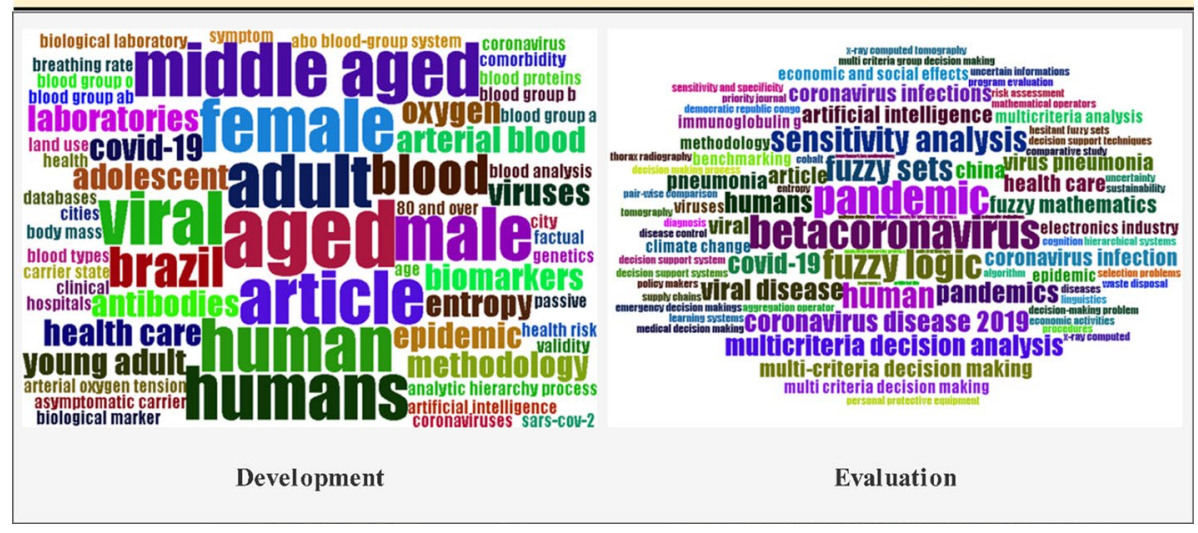

Fig. 5 Keyword Plus co-occurrence network 


\subsubsection{WordCloud}

Coword analysis aims to develop a conceptual framework by mapping and clustering phrases retrieved from bibliographic metadata such as keywords, titles and abstracts, as illustrated in Fig. 5.

Wordcloud can support research taxonomy by dividing MCDM and COVID-19 studies into two categories. Figure 5 shows that in the evaluation and development category, the most relevant parts of the textual data were organised. This procedure helped academics and beneficiaries compare the different parts to find similarities and differences amongst the larger, bolder word. Moreover, Wordcloud has provided the opportunity for academics and industry to understand the importance of adopting MCDM methods with COVID-19 by clustering of words depicted in different sizes.

\subsubsection{Factor analysis in bibliographic data}

Based on the factorial analysis, the similarity function may cluster bibliographic coupling, cocitation and co-occurrence data (Aria and Cuccurullo 2017). The benefits of adopting the similarity function to compute proximity index, Jaccard's coefficient, inclusion index, and Salton's cosine are shown in Fig. 6.

Figure 6 illustrates the factorial analysis for the development and evaluation category. The keywords for each category (i.e. evaluation and development) were divided into two clusters based on similarity index. The adoption of this function based on the evaluation and development category contributed extensively to discovering relevant knowledge from literature regarding MCDM methods with COVID-19. Furthermore, the similarity index is considered high due to the similarity of the issues studied amongst the reviewed literature. The similarity function provides the homogeneous clusters of related studies that may support the academics and beneficiaries to distinguish and recapitulate the relevant information.

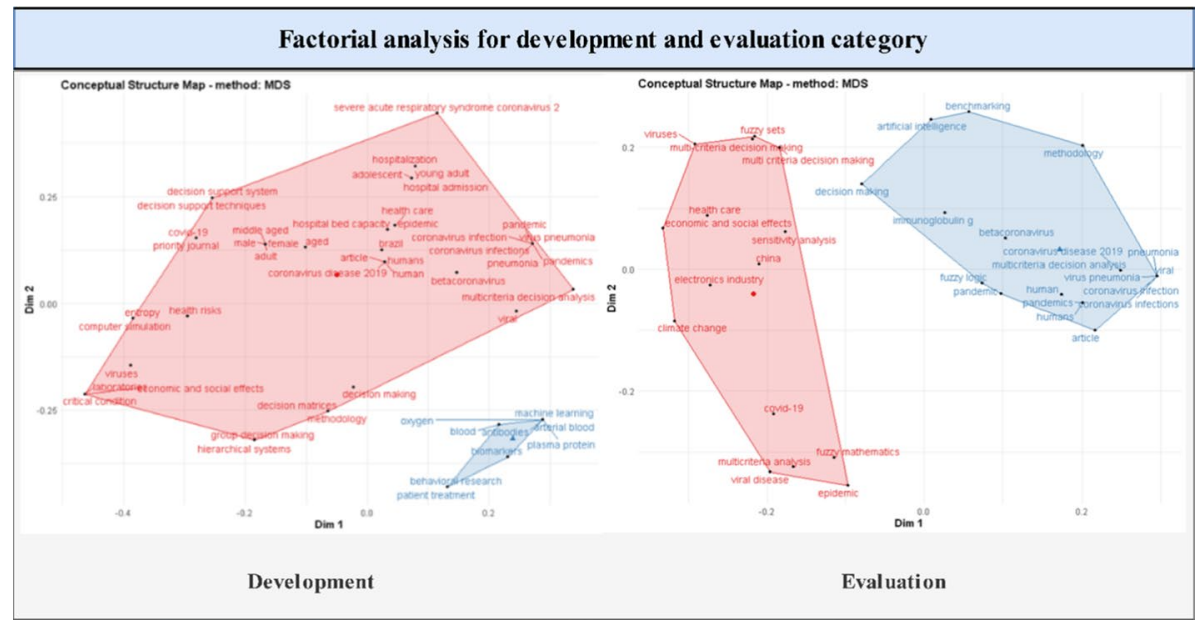

Fig. 6 Conceptual map and keyword clusters 


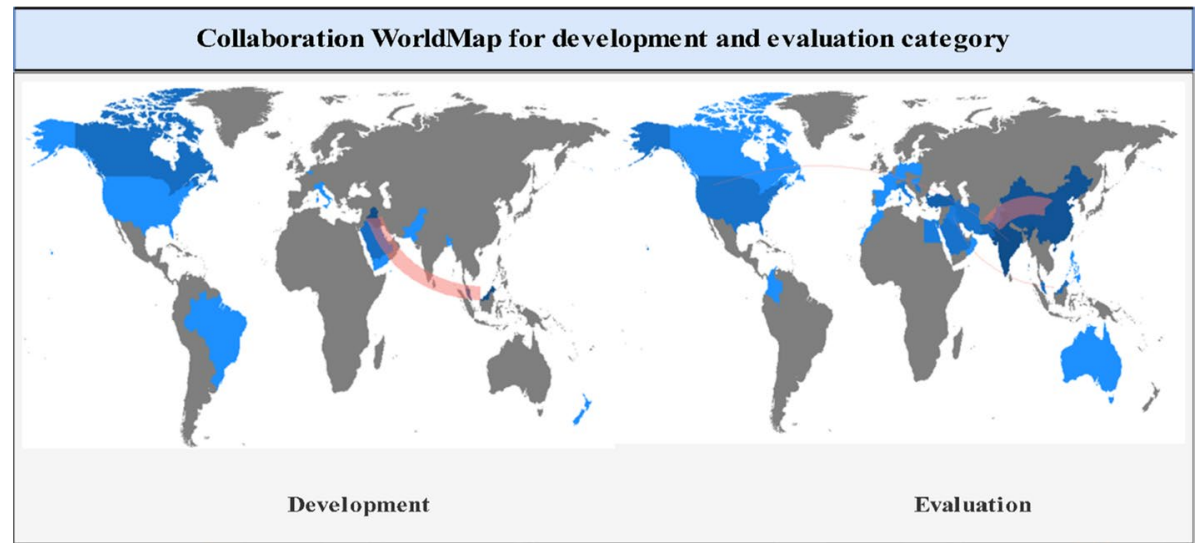

Fig. 7 Country collaboration map

\subsubsection{Country collaboration map}

Authors and their coauthorships form the foundation of a scientific cooperation network. Scientific cooperation is one of the most well-documented types of collaboration in the sciences (Glänzel and Schubert 2004). Figure 7 depicts a map of the scientific collaborations of authors located in other countries.

For the evaluation category, Asian-American cooperation and European-African cooperation are lacking. Consequently, this gap may constitute potential future studies that seek to solve COVID-19 problems based on the MCDM methods. However, the development category has a widespread lack of scientific collaboration regarding MCDM techniques and COVID-19. Hence, the MCDM-COVID-19 issue research network explained the existence of a lack the scientific cooperation between authors regarding the development category.

\subsection{Criteria standardisation}

DM problem in the context of COVID-19 is affected by multiple criteria and influence the final DM results. In literature, defining and collecting main criteria and sub criteria set implemented the following different approaches. The research work from both perspectives (i.e. evaluation and development) was analysed based on whether the defined main criteria or sub criteria followed any direction of standardising during collection and what the standard followed was, as shown in Table 3.

Table 3 shows two categories conducted for a consideration of collected information in the table organisation. One came from the category of evaluation, and the other came from development. However, each came out with different percentages when matching the literature studies in terms of the main and sub criteria, the direction of standardisation for main and sub criteria, and what standardised method has been followed for acquiring the collected criteria. These percentage results are discussed as follows:

For the evaluation category The main criteria were definitely utilised in the entire articles to be included and obtained $100 \%$ whilst not all articles included sub criterial; thus, the percentages here were assigned (26.82\%). Accordingly, the majority of standardisation directions for the main and sub criteria had wider differences and can be recognised in 


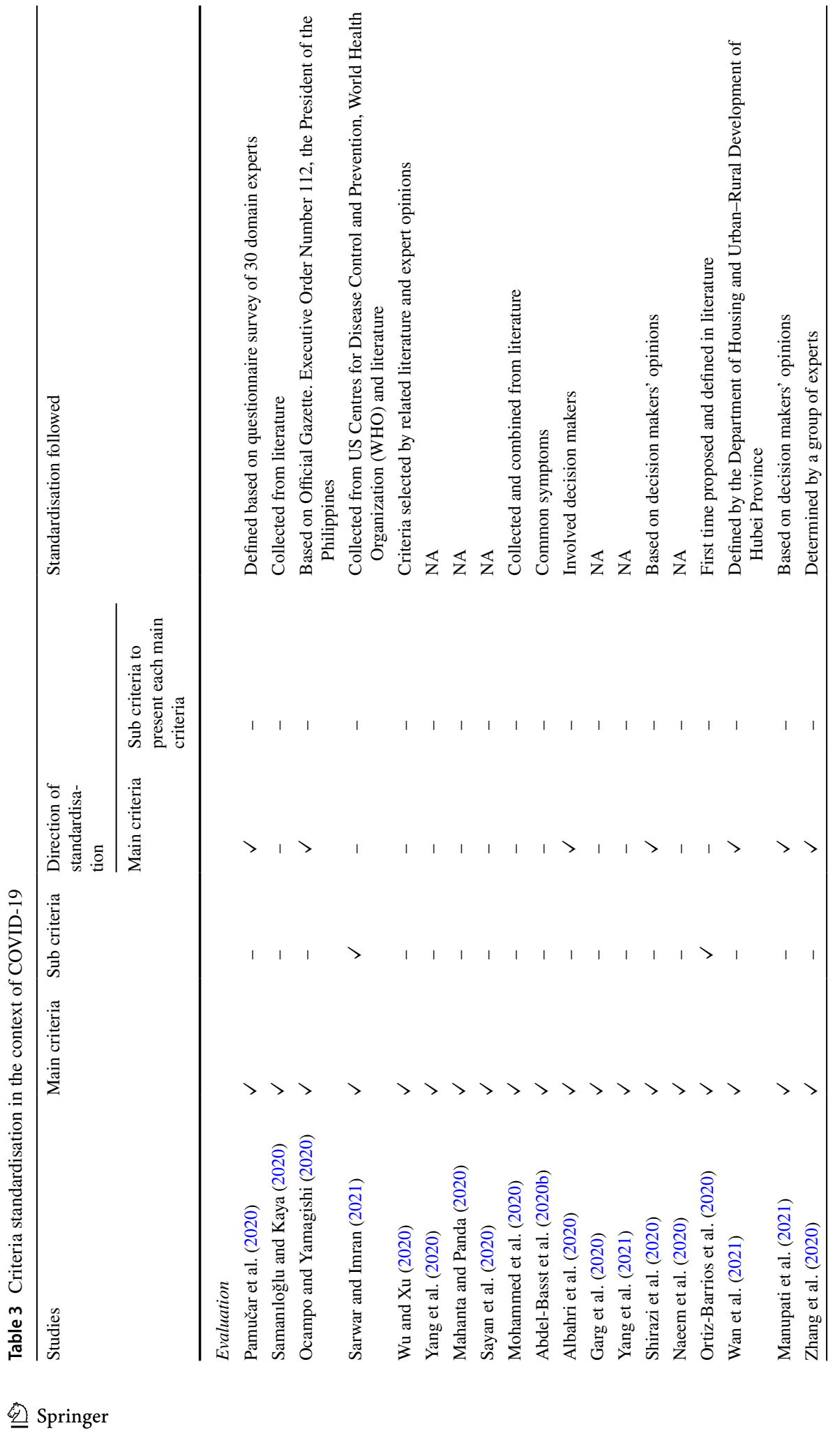




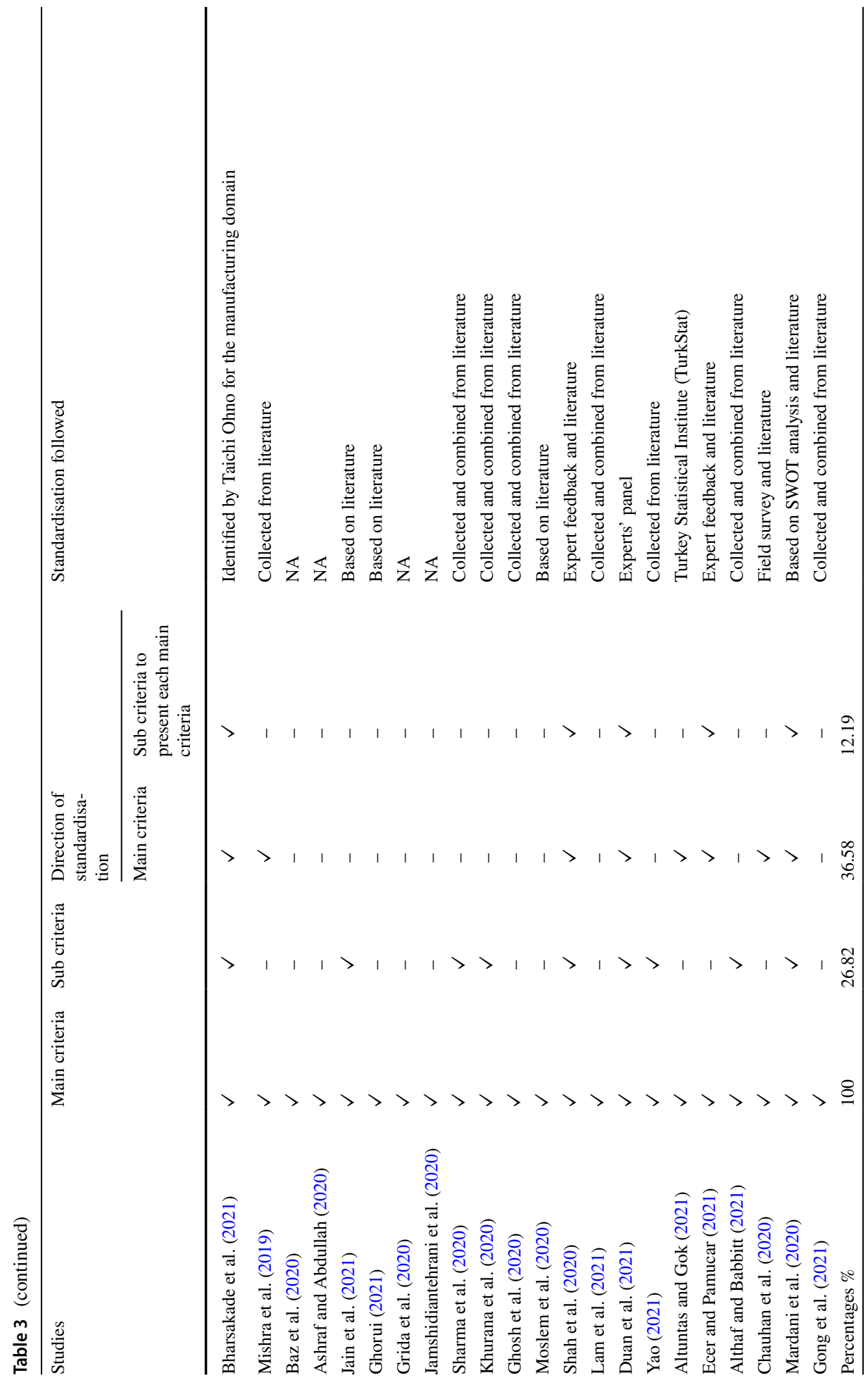




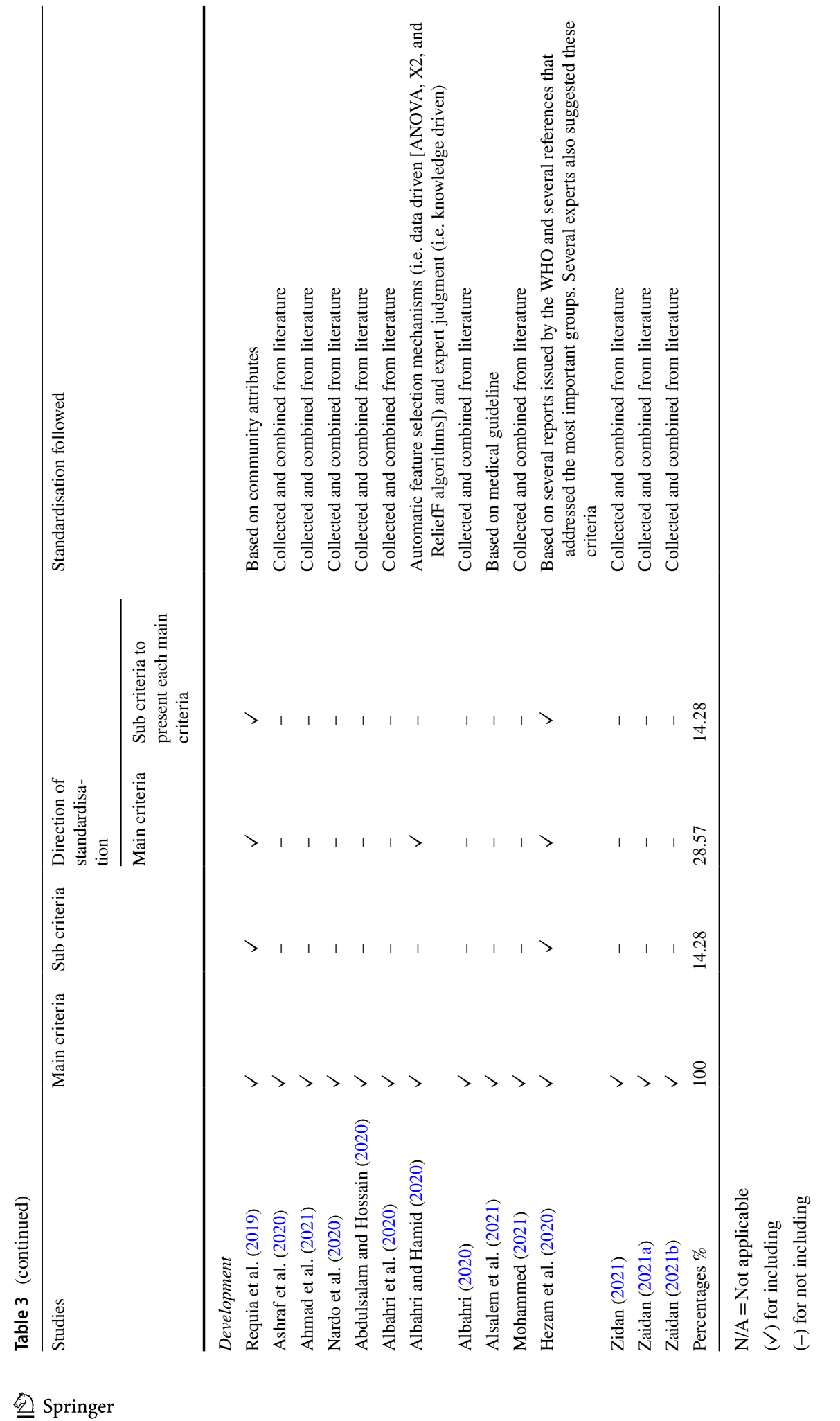


terms of percentage results. For the most part, the unexpected results is that few studies (36.58\%) used guidelines to develop a clear and/or standard plan for evaluating the main criteria of COVID-19 in different case studies. This conclusion became clearer when a lower percentage of $(12.19 \%)$ was found towards the trend of standardising the sub criteria. The last column that was considered the most important part of the evaluation category to be discussed, in which how the studies justified the standardisation followed for their utilised criteria was explained, in particular, suffered from a paradox. The findings showed $(10,24.39 \%)$ out of 41 studies in this column missed this part and did not even mention the conducted standards followed. This result leads us to ask a question whose answer may also be missing: how can the scholars verify that this is the fitting grouping of criteria. If one criterion was missed in the case study, mostly the developed methodology, and even the obtained results for this study could be different. Fourteen studies justified the standard followed and the criteria used from the literature. Occasionally, this standard direction does not confer a respectable reason of the criteria; however, it may be somewhat acceptable for case studies. The 16 remaining studies followed different standard directions such as statistical method, survey, organisation and/or report based, and used expert opinion towards the collection criteria, and several studies used a method combined with the literature for accompanying the used criteria. Nevertheless, these kinds of studies are still confused in terms of no clear justification and guideline for the criteria types and numbers.

For the development category This category had more missed information in the above columns than the evaluation category, in which this discussion part becomes more argumentative. In this category, the standardisation of the criteria is a crucial part with respect to the DM method when providing services is necessity in the context of COVID-19, especially for the studies focused on the medical treatment for patients and hospital services. This category contained 14 studies, each had main criteria (100\%) and only two articles (14.28\%) had sub criteria when achieving the development. For the standardisation directions, only 4 out of 14 articles (28.57\%) directed their way of standard direction concerning the main and only 2 articles (14.28\%) for sub criteria. For the last column, (10, 71.42\%) out of 14 studies justified that they utilised the criteria from the literature in terms of the standard followed when developing their works, and 4 studies had different directions such as community attributes-, automatic feature selection-, medical guideline- and organisationbased, each of which was conducted by only one study.

In conclusion for both categories, despite the various methods of evaluating the criteria of COVID19 amongst the 55 studies, no approach was presented as an agreement or justification method about how to collect, unify and standardise the criteria and sub criteria of COVID19 (i.e. types and numbers), and how it can be verified. In terms of evaluation or developing works in different COVID19 case studies, these issues should be considered and solved.

\subsection{MCDM methods}

The techniques of MCDM are theoretically divided into two directions: ranking methods, which are used to rank the alternatives, and weighting methods, which are used to compute the importance level of each criterion. The MCDM in the evaluation and development perspectives employs various methods in handling COVID-19 issues. A comprehensive analysis for all these MCDM methods (either ranking or weighting methods) is presented with their originality (i.e. existing, new or extension) and the applied fuzzy environment in Table 4. 


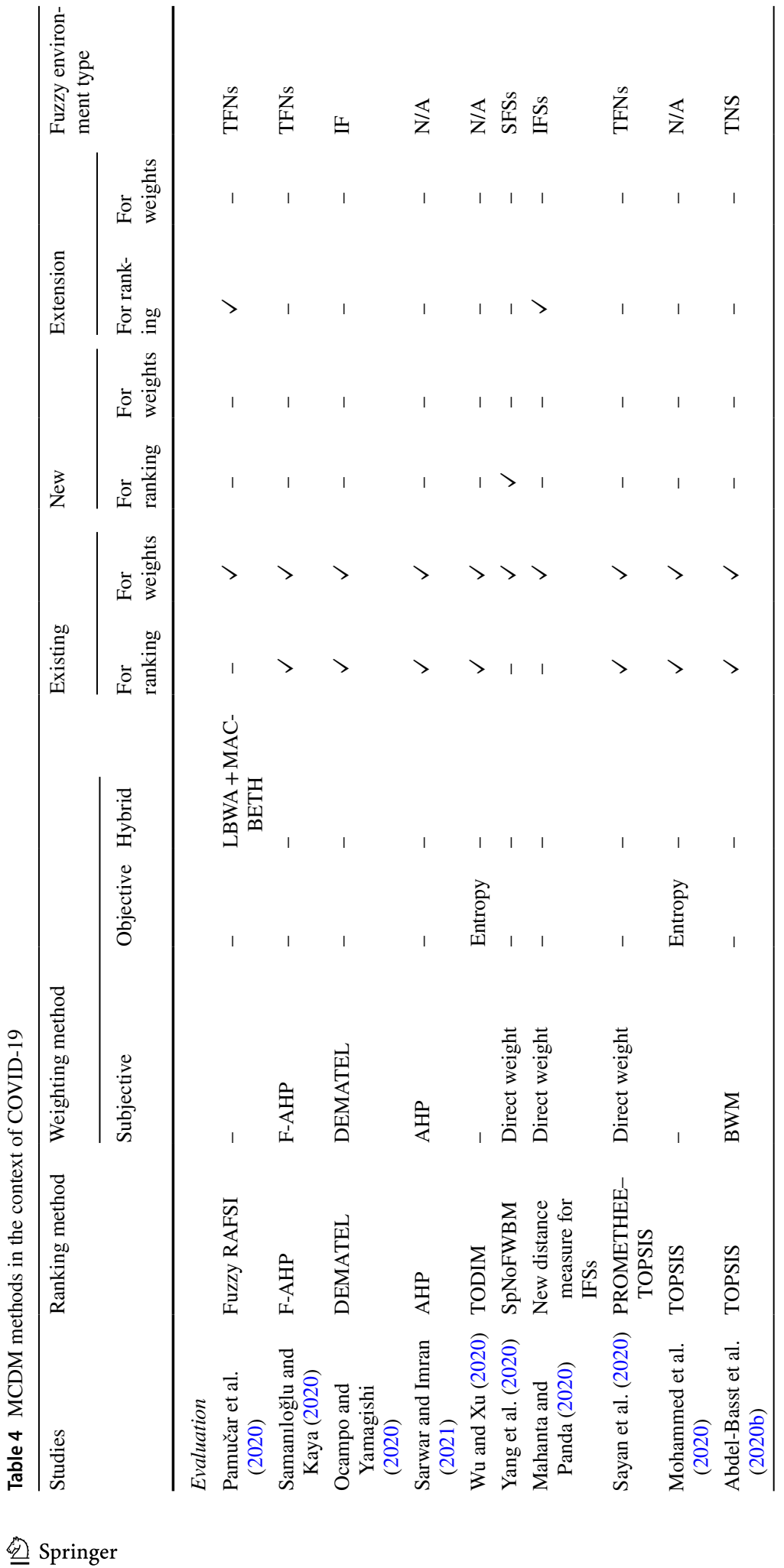




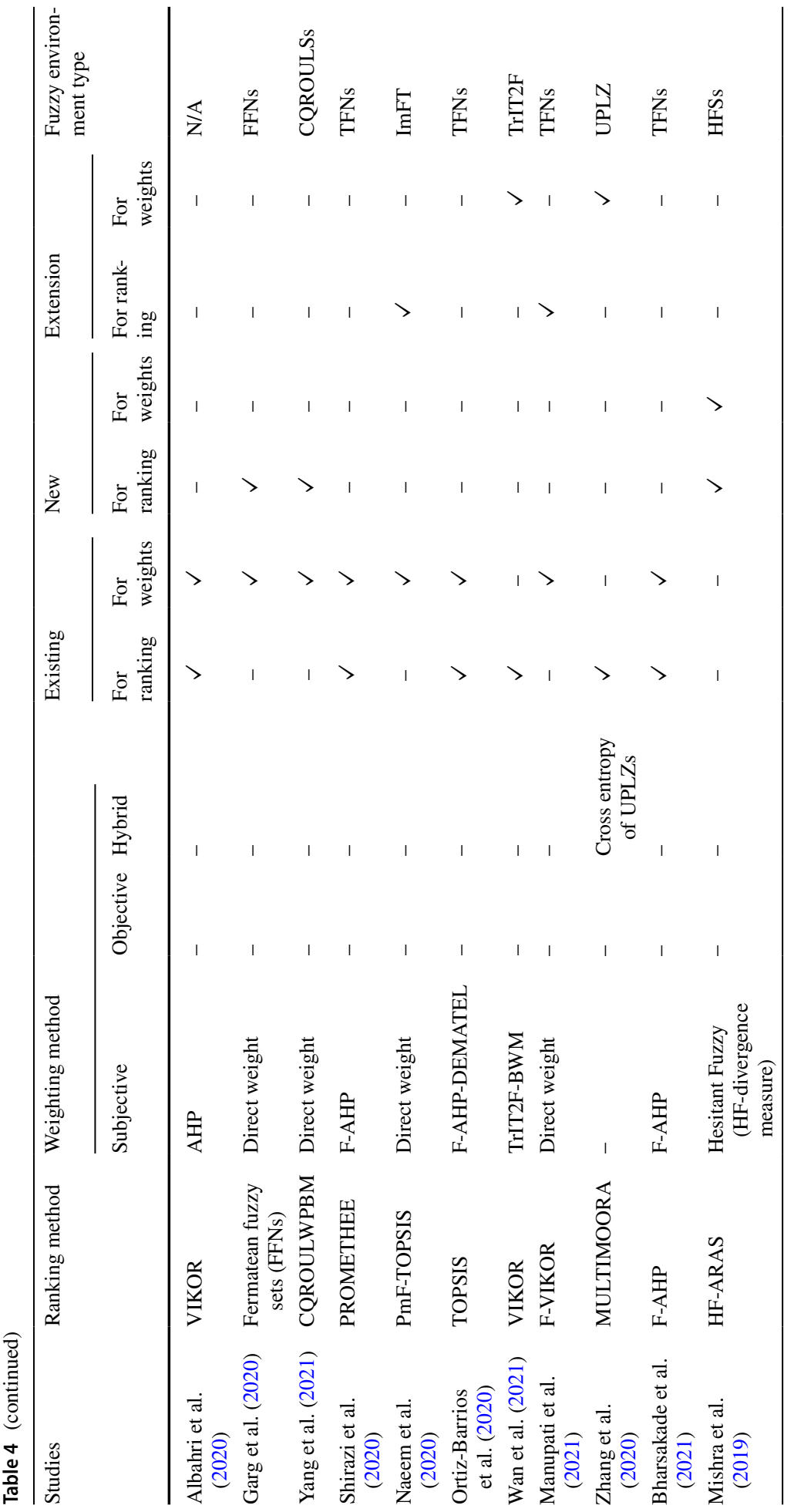




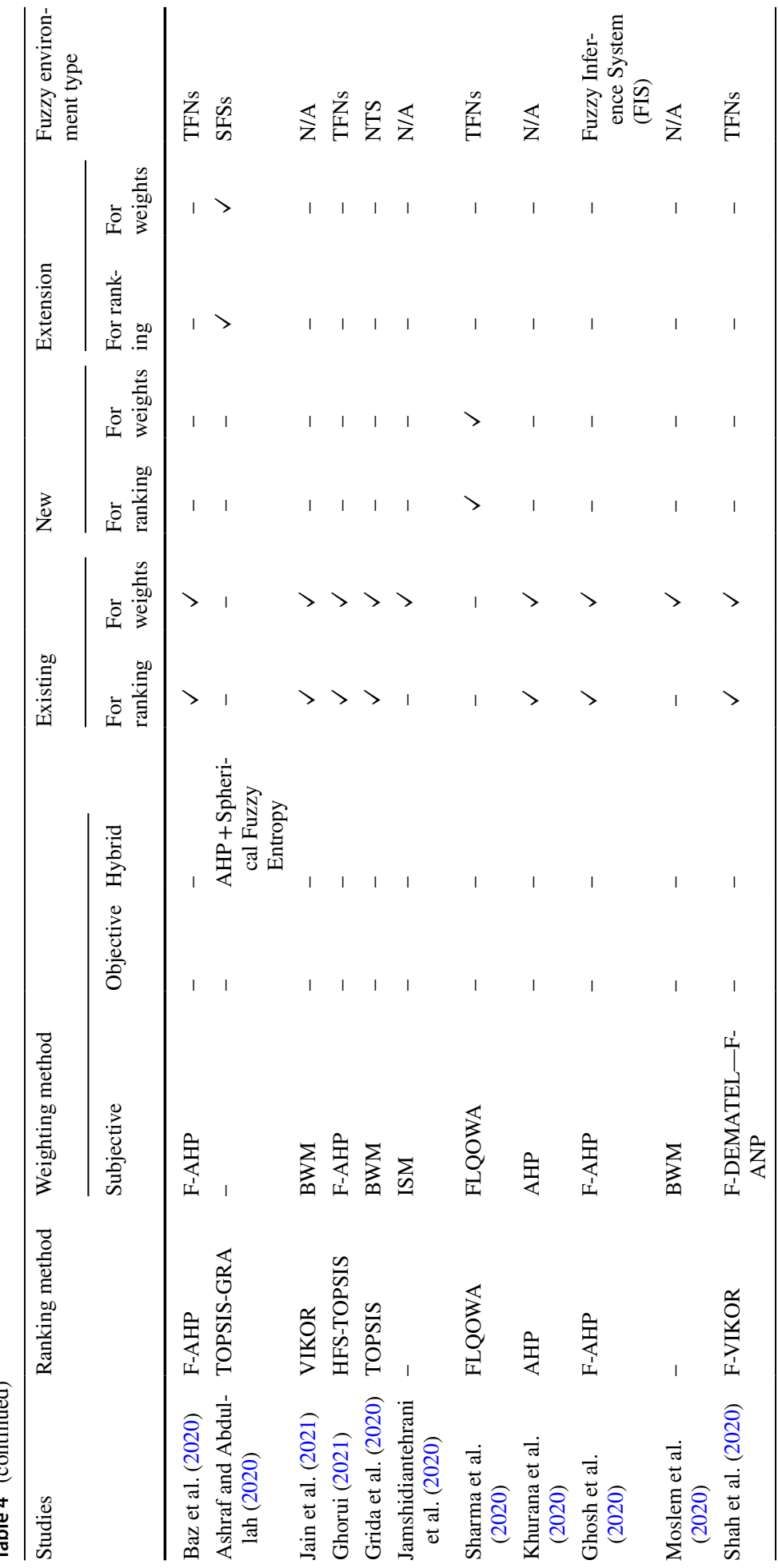




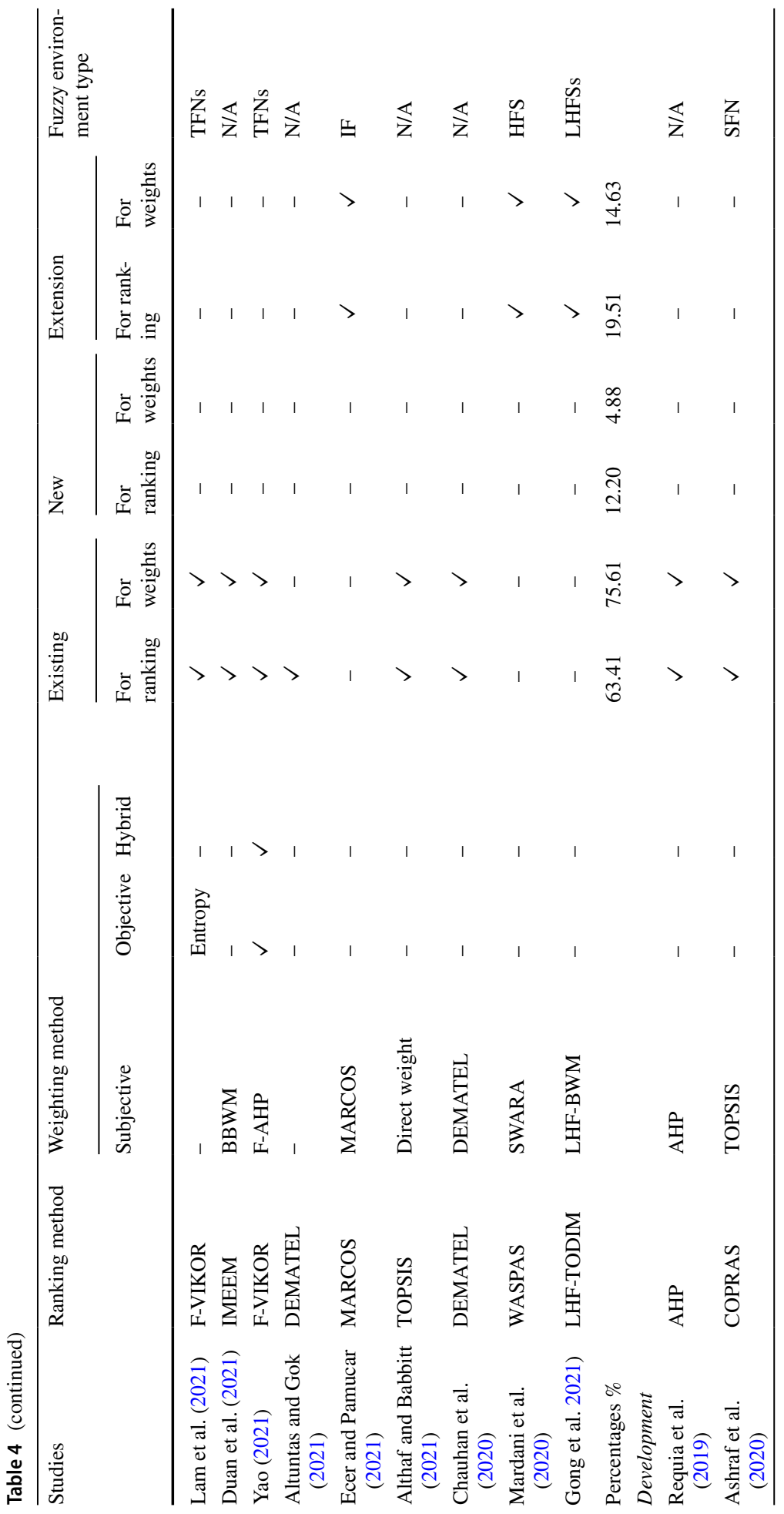




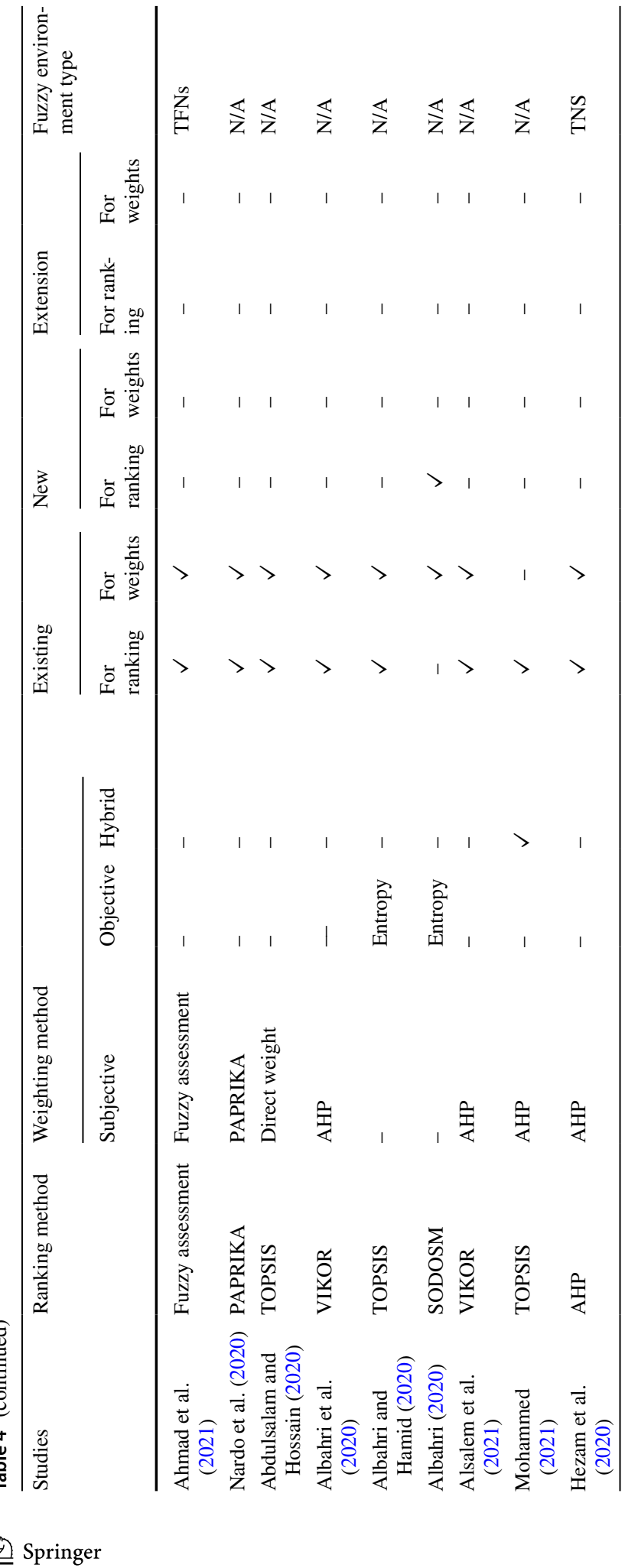




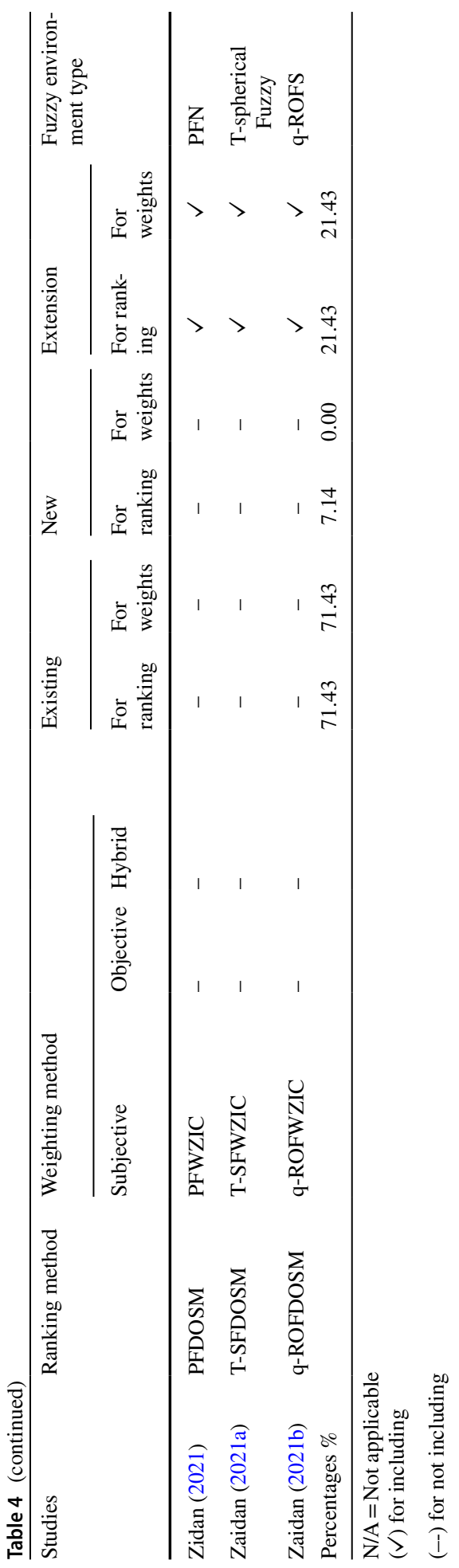


The collected information in Table 4 confer the state-of-the-art ranking and weighting methods used in COVID-19 studies in terms of evaluation and development. The discussion of this table can be explained as follows:

For the evaluation category Different ranking methods have been used in this category that considers the required prioritisation for handling COVID-19 case studies. The most frequently used is the TOPSIS method $(9,21.95 \%)$ out of 41 , followed by 6 used for each of VIKOR and AHP methods, followed by 3 used for DEMATEL method while the others have been used in a limited manner. However, most of ranking methods have been integrated with other weighting methods to complete the aim of those evaluation studies. If argument is desired on weighting methods' column, three types of methods will be found: subjective, objective and hybrid. For the subjective methods, $(11,26.82 \%)$ out of 41 used the AHP method, followed by $(8,19.51 \%)$ out of 41 studies for direct weight, followed by 7 studies for BWM, followed by 4 studies for DEMATEL, and the other methods had a limited use. Moreover, a very limited use was seen for objective and hybrid weighting methods that were assigned only 3 out of 41 studies each. For ranking and weighting methods, three important perspectives are shown in Table 4 to provide a clear evidence for answering the question of how many studies in the literature (in terms of percentages \%) utilised existing methods, conducted new methods and went far to extend new methods. Thus, high percentages were obtained by the existing ranking and weighting methods with percentage values of $63.41 \%$ and $75.61 \%$, respectively. The extension ranking and weighing methods obtained $19.51 \%$ and $14.63 \%$, respectively, whereas the low percentages obtained by the new ranking and weighting methods were $12.20 \%$ and $4.88 \%$, respectively. These percentage results indicate research conducted existing methods for evaluation COVID-19 case studies rather than focusing on new or extended methods. Finally, for the last column on fuzzy environment types $(12,12.26 \%)$ out of 41 studies did not consider any fuzzy type, which, to our knowledge, needs to be reconsidered because fuzzy DM aims to select the best alternative in case of imprecise, incomplete and vague data that need to be applied with COVID-19 case studies according to its relevance. However, the 29 other studies used different fuzzy types, in which the TFNs was the most frequently used with $(13,31.70 \%)$ out of 41 studies.

For the development category This category had different percentage results, especially for the limited cases for the development of new ranking and weighting methods. For the ranking methods, TOPSIS was the most frequently used with $(3,21.42 \%)$ out of 14 studies whilst other ranking methods had limited use. For the weighting methods, AHP was the most used with $(5,35.71 \%)$ out of 14 studies within the subjective weighting methods. In objective weighting methods, entropy was used with $(2,14.28 \%)$ out of 14 studies, and no hybrid weighting methods were adapted under this category based on the literature. The existing ranking and weighting methods were used in the literature and obtained $71.43 \%$ and $71.43 \%$, respectively. The new ranking method was developed and obtained $7.14 \%$. No new weighting method was developed for the COVID-19 case study $(0.00 \%)$. Finally, the ranking and weighting methods were extended in the literature and obtained $21.43 \%$ and $21.43 \%$, respectively. For the fuzzy environment types, only $(6,42.85 \%)$ out of 14 studies were used, whereas other studies were not applicable (N/A), in which the fuzzy environments were fairly used within the development category. 


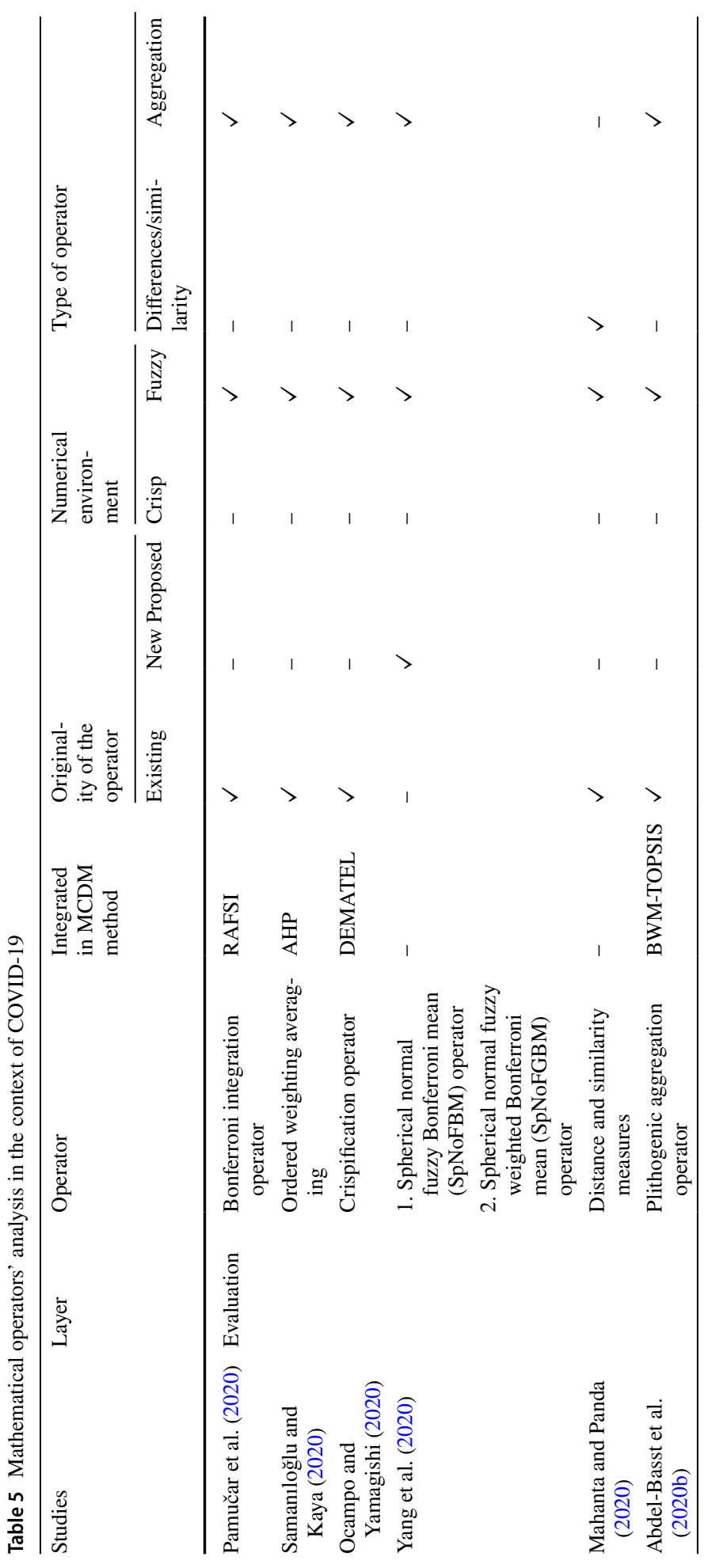




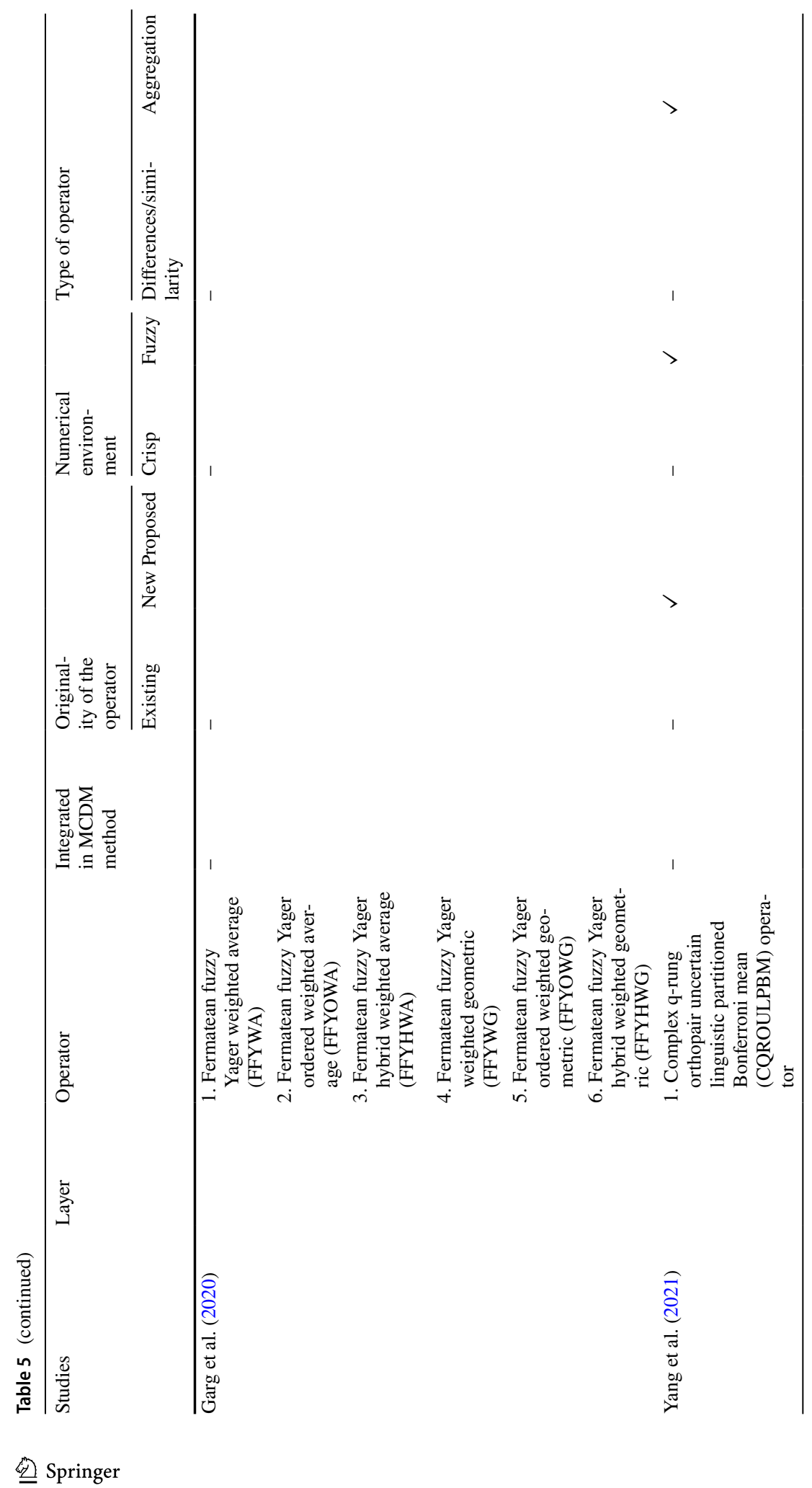




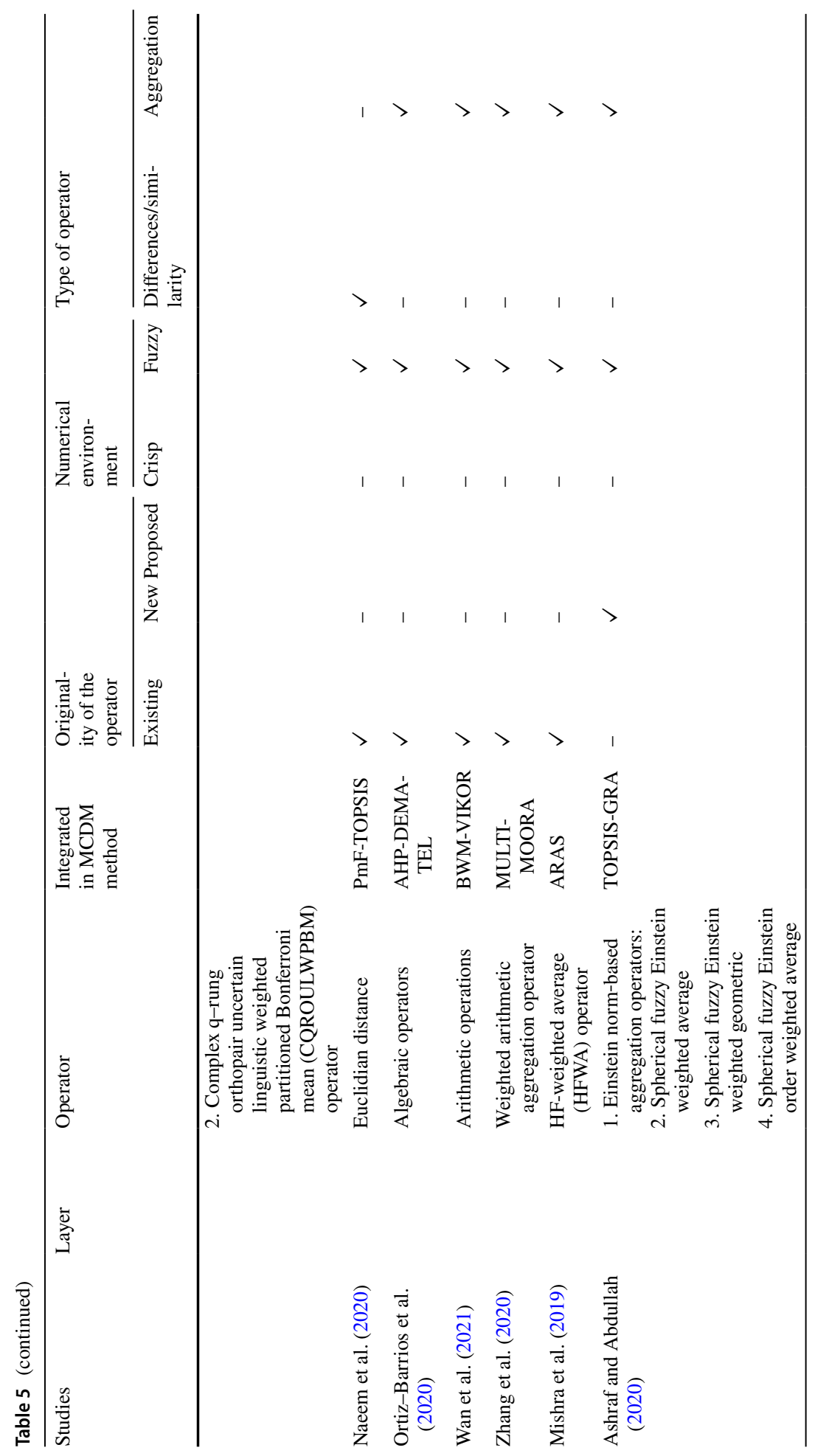




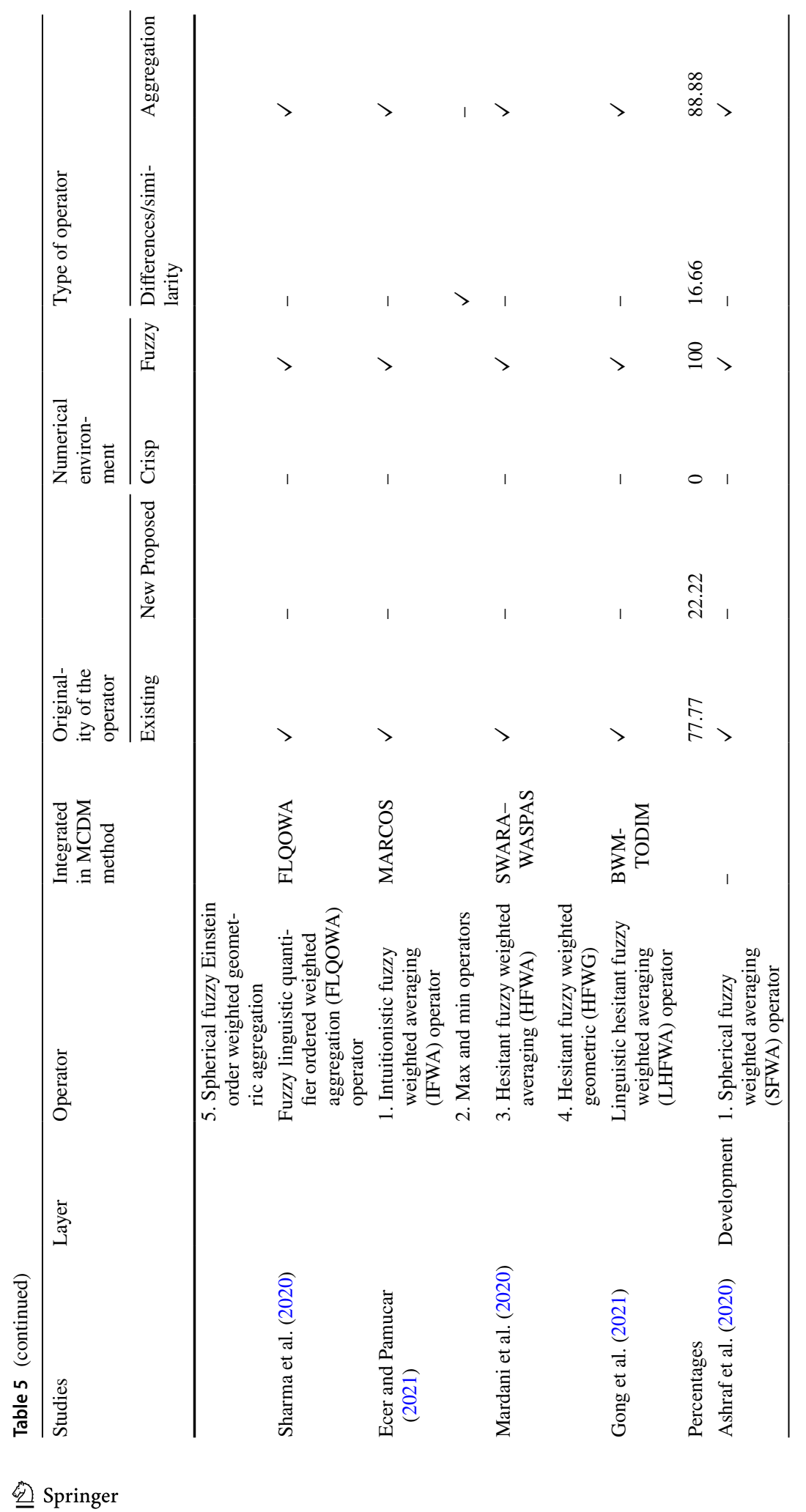




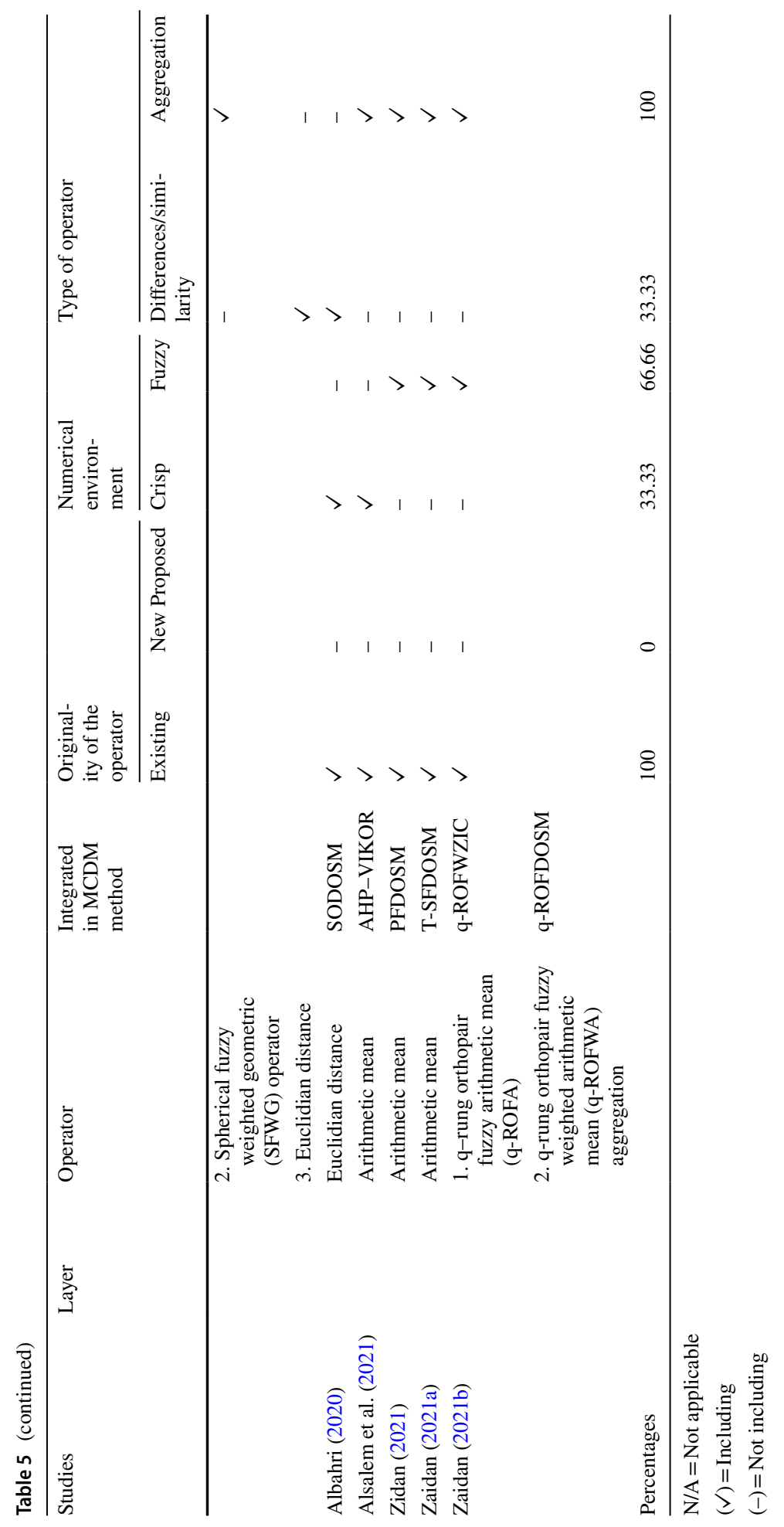




\subsection{Mathematical operators}

In MCDM theory, different operators are integrated and used in COVID-19 studies. Each of these operators followed a specific mathematical model that might have different effects on the aggregation or distance computation of the final DM outcome. Thus, demonstrating the integrated operator and its types could provide better understanding on the final results differences. An analysis was implemented to explain the integrated operators used with MCDM of the COVID-19 studies, the originality of the operator (i.e. existing, or new), the adapted numerical environment (i.e. crisp, or fuzzy) and the operator types (differences/ similarity or aggregation), as shown in Table 5.

In Table 5, different types of operators were used in the literature with various MCDM methods in the contexts of COVID-19 case studies. This table shows the theoretical aspects of how these operators were distributed in terms of utilisation amongst the two categories. Clearly, the most operators are being used within the evaluation category because the number of studies within this category are more than the development category and can be discussed as follows:

For the evaluation category, $(14,77.77 \%)$ out of 18 studies integrated MCDM methods with different types of operators, in which three of these works utilised more than one operator. In particular, the study of Ashraf and Abdullah (2020) used four types of operators with the integration of TOPSIS-GRA methods, the study of Mardani et al. (2020) used two types operators with the integration of SWARA-WASPAS methods and the study of Ecer and Pamucar (2021) used two types of operators with the MARCOS method. This method refers to the application of fuzzy environment that requires a special treatment to handle the fuzzy information in the context of DM for COVID-19 studies. On the contrary, 4 out of 18 studies (Yang et al. 2020, 2021; Mahanta and Panda 2020; Garg et al. 2020) used different operators without integrating any of the MCDM methods because they applied a direct ranking of the aggregation results. In addition, the originality of the operators is presented in Table 5 with two main aspects (i.e. existing and new proposed). High percentages were obtained by utilisation of an existing operators $(77.77 \%)$ rather than a new proposed $(22.22 \%)$. Especially, all these existing operators were applied in fuzzy environments $(100 \%)$ where no study $(0 \%)$ conducted a crisp numerical environment. For the last column in the evaluation category, the operators can be classified into two main types: differences/ similarity and aggregation. The aggregation was highly used in the literature with $(88.88 \%)$ percentage of studies with (16.66\%) assigned to the differences/similarity type.

For the development category, 5 out of 6 studies used different MCDM methods with numerous operators, and only one study used direct ranking, following the same reason mentioned in the evaluation category. The most frequently used was the arithmetic mean operator (3 studies) (Alsalem et al. 2021) (Zidan 2021; Zaidan 2021a). All operators in this category considered an existing operator (100\%), and no new one was proposed $(0 \%)$ within the development studies. The percentage use of fuzzy was more than that of crisp and obtained $(66.66 \%)$, whereas crisp obtained $(33.33 \%)$ results. For the type of operators, the aggregation was mostly used with (100\%) whilst the differences/similarity was obtained (33.33\%).

\subsection{Data normalisation}

One of the main issues that might affect the MCDM methods is the employed normalisation technique. Most MCDM methods implement normalisation techniques to produce scaleless 


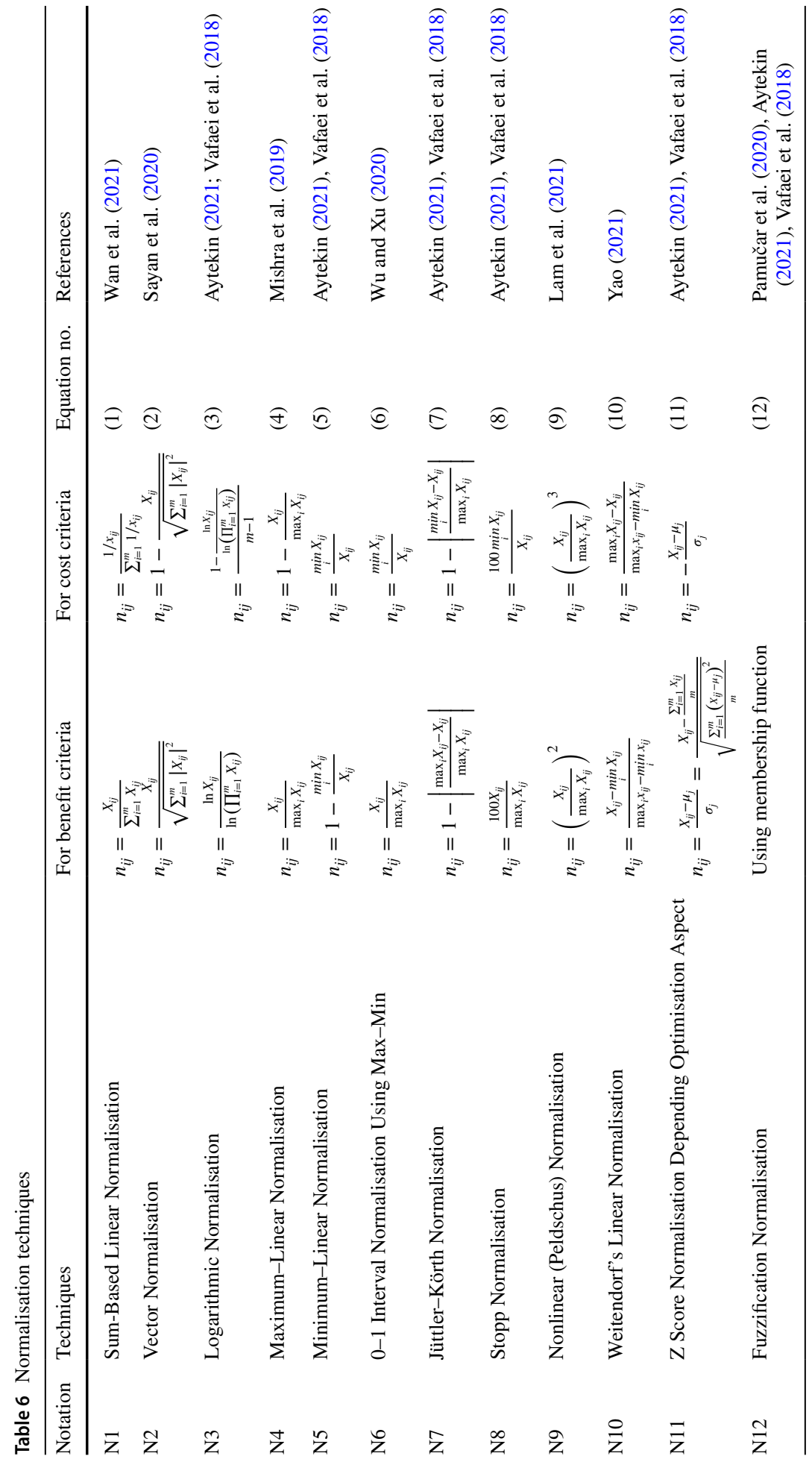




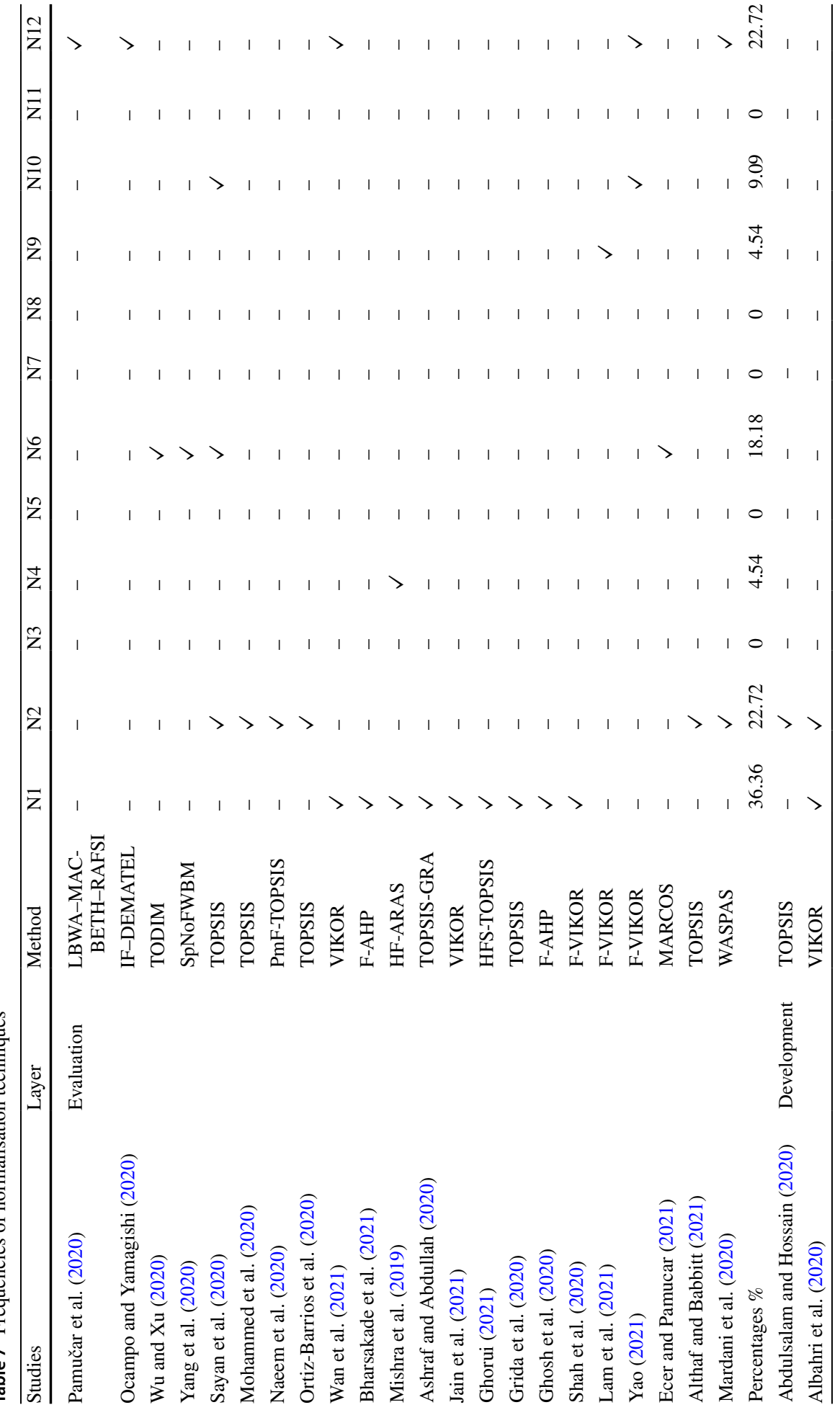




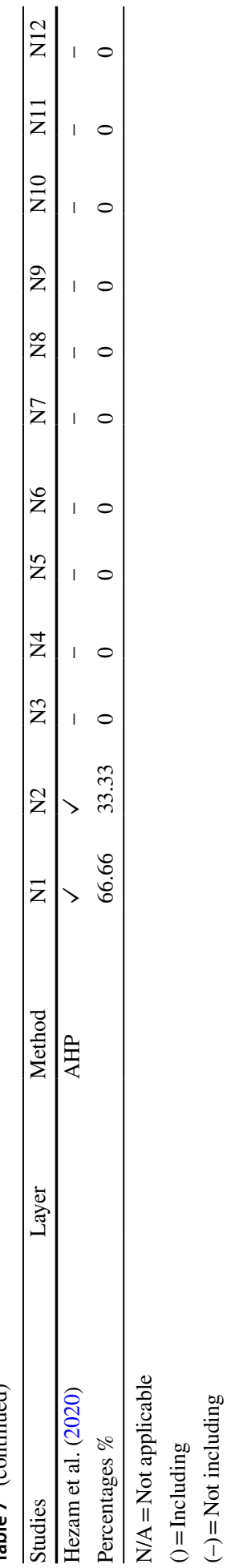


data to aggregate/rank alternatives. Using different normalisation techniques may lead to different rankings. Based on literature, 12 normalisation procedures are used diversely and widely within the MCDM methods (Aytekin 2021; Vafaei et al. 2018). Table 6 lists all employed normalisation techniques for cost and benefit criteria. Consequently, studying the normalisation type and its frequency used in literature of COVID-19 is necessary in understanding the difference in ranking results. The distribution of frequency used for each of these normalisation techniques within MCDM in the context of COVID-19 is presented in Table 7 .

The distribution of frequency use of normalisation techniques within MCDM in the context of COVID-19 is demonstrated in Table 7.

Twelve normalisation methods (N1-N12, as shown in Tables 6 and 7) are used for correcting experimental variation (different scale) and bias in data to be used with MCDM methods. However, Table 7 shows limited conducting for several normalisation methods and lack of use for others. For evaluation category (22 studies), up to 5 normalisation methods (N3, N5, N7, N8, and N11) amongst them were not used for any MCDM method within COVID-19 case studies. Others (N1, N2, N4, N6, N9, N10 and N12) were used with limited percentages of $36.36 \%, 22.72 \%, 4.54 \%, 18.18 \%, 4.54 \%, 9.09 \%$ and $22.72 \%$ respectively. N1 was the most dominated normalisation method amongst others with 8 studies. Compared with the evaluation category, the development category included only three studies, in which several normalisation methods were rarely utilised. Thus, 8 normalisation methods were not used in the literature and obtained 0 percentages for each of (N3-N12). Only N1 and N2 were used with $66.66 \%$ and $33.33 \%$ respectively. In conclusion, the COVID-19 case studies and summarisation of these normalisation methods shed light on understanding the appropriate normalisation method amongst others when used with MCDM methods. Similarly, the limited used of normalisation methods within these two categories led to a minimal effect on the scaling of COVID-19 data. Overall, the influence of normalisation methods in the context of COVID-19 case studies integrated with MCDM methods were not as much as acquired sufficient attention for determining the best normalisation analysis.

\subsection{Decision-making contexts}

Aggregating the final weight values in the decision matrix and decision information were implemented differently in literature. The aggregation procedure of the decision information in the context of COVID-19 studies were analysed. Two procedures were used, namely, individual and group. In individual procedure, the weight values were applied on each decision matrix of each expert individually before aggregating and computing the final rank. In group procedure, the focus was on the decision matrix aggregation and ranking, which also had two sub procedures, namely, internal (if the aggregation of all decisions matrices was implemented before computing the final rank) or external (if the aggregation of all decisions matrices was implemented after computing the final rank per matrix). The distribution of frequency used of each of these procedure within MCDM in the context of COVID-19 is presented in Table 8.

Table 8 illustrates how the MCDM methods were used with respect to individual and group DM contexts. This part of the analyses is very important because it highlights the ranking context types to promoting prioritisation practice (i.e. individual or group) for better control of COVID-19 disease. For evaluation category, the internal group context was widely applied in 33 out of 42 studies and obtained $78.57 \%$, whereas the external group 


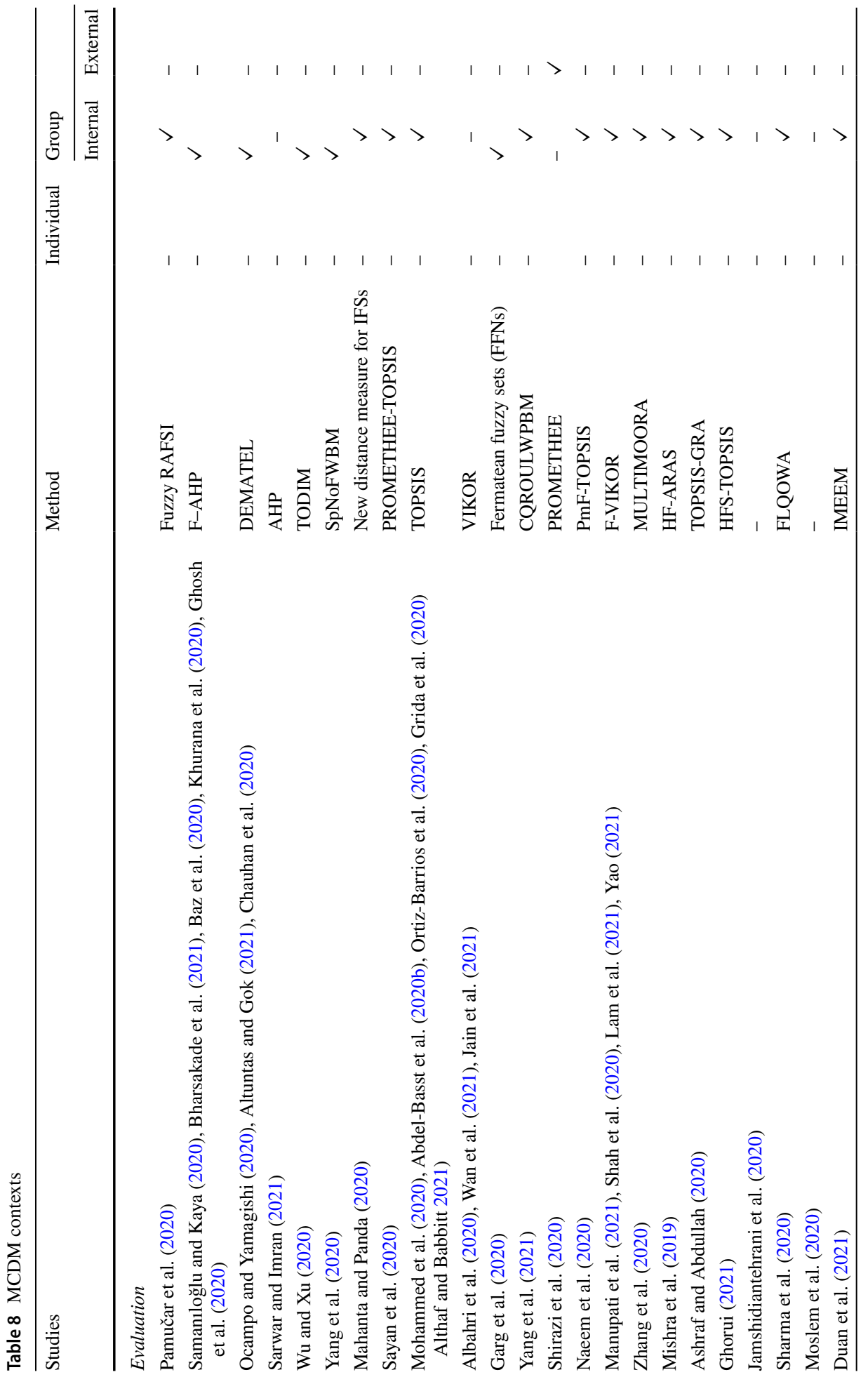




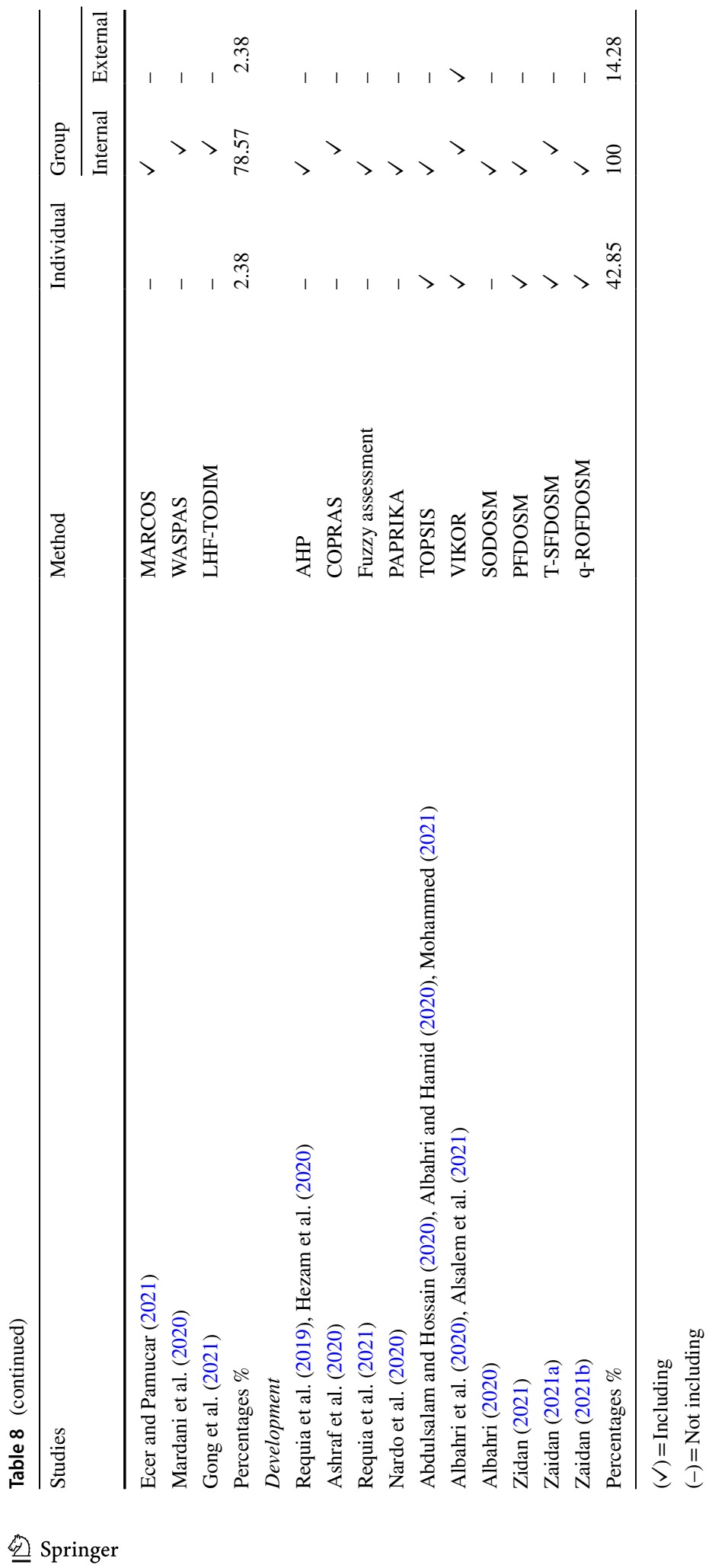


context was applied only in one study and obtained $2.38 \%$. Similarly, the individual context was applied in one study and obtained $2.38 \%$. For the development category, 6 out of 14 studies applied individual context and obtained $42.85 \%$. The internal group context was applied in all studies, obtaining $100 \%$, followed by external group context that was applied in 2 out of 14 studies and obtained 14.28\%. However, arguments for and against these two contexts are presented and discussed this table to conclude that nonbalance was conducted on COVID-19 research progress with respect to both. The internal group DM context acquired more attention to be applied within the two categories.

\subsection{Experts' selection}

In DM, the expert or decision maker has a main contribution in most of the MCDM processing stages and their feedback, and consensus influences the ranking results and final decision (Mohammed et al. 2021b). Consequently, understanding the selection methods, selection criteria and the number of participated expert experts is incredibly important. The type of expert selection method, the condition of expert selection and the number of experts in the context of COVID-19 were analysed, as presented in Table 9.

The information in Table 9 explains to the researchers how the experts were selected by the authors of the above studies. Although the context of the above studies is one case, a study COVID-19 belonging to the family of respiratory diseases, the experts who were selected were clearly from various fields, not only from respiratory fields because the direction fields of these studies were virology, infectious diseases, ICT, pneumology physicians based in emergency, academia, research institute, economist and ecologist. For the two categories, these frequent limitations were found in most of studies regarding the type of guideline method for selecting the experts, of which the majority were not mentioned in studies. However, several studies (such as (Shirazi et al. 2020; Chauhan et al. 2020)) mentioned the conducted method either as a sample size of population, followed a guideline method for panel experts' selection or others. Three experts were selected in most studies with more than 10 years of experience domain condition as the most frequently acceptable number of experts in the literature in neuroses fields in the context of COVID-19.

\subsubsection{MCDM validation scheme}

The validity of the MCDM method in terms of appropriateness and suitability plays a vital, critical role in the accuracy of the final decision, especially in the context of COVID-19, where the yield of DM involves human life from different facets. In literature, most of the research work, either evaluation or development perspective, focused mainly on evaluating the MCDM outcome using different techniques, such as comparison analysis: Different MCDA methods/models present different models of decision making/analysis. Thus, possible distinctions in ranking by different models are natural. If the situation with alternatives is robust enough, different models (can) result in the same ranking. However, for example, if the "best" alternative, A1, is robust for MCDA method MAVT, and the "best" alternative, A2, is robust for TOPSIS, it doesn't mean A1=A2. There can be several different fuzzy extensions of ordinary MCDA methods. E.g., Fuzzy TOPSIS methods can differ only by ranking methods. According to some new results, the probability that ranking alternatives by these two methods can be different is high/significant. Thus, Comparative analysis for such cases can be ineffective/unjustified (Pamučar et al. 2020); sensitivity analysis: (tweaking the parameters): tests the influence of changing parameters on the decision 


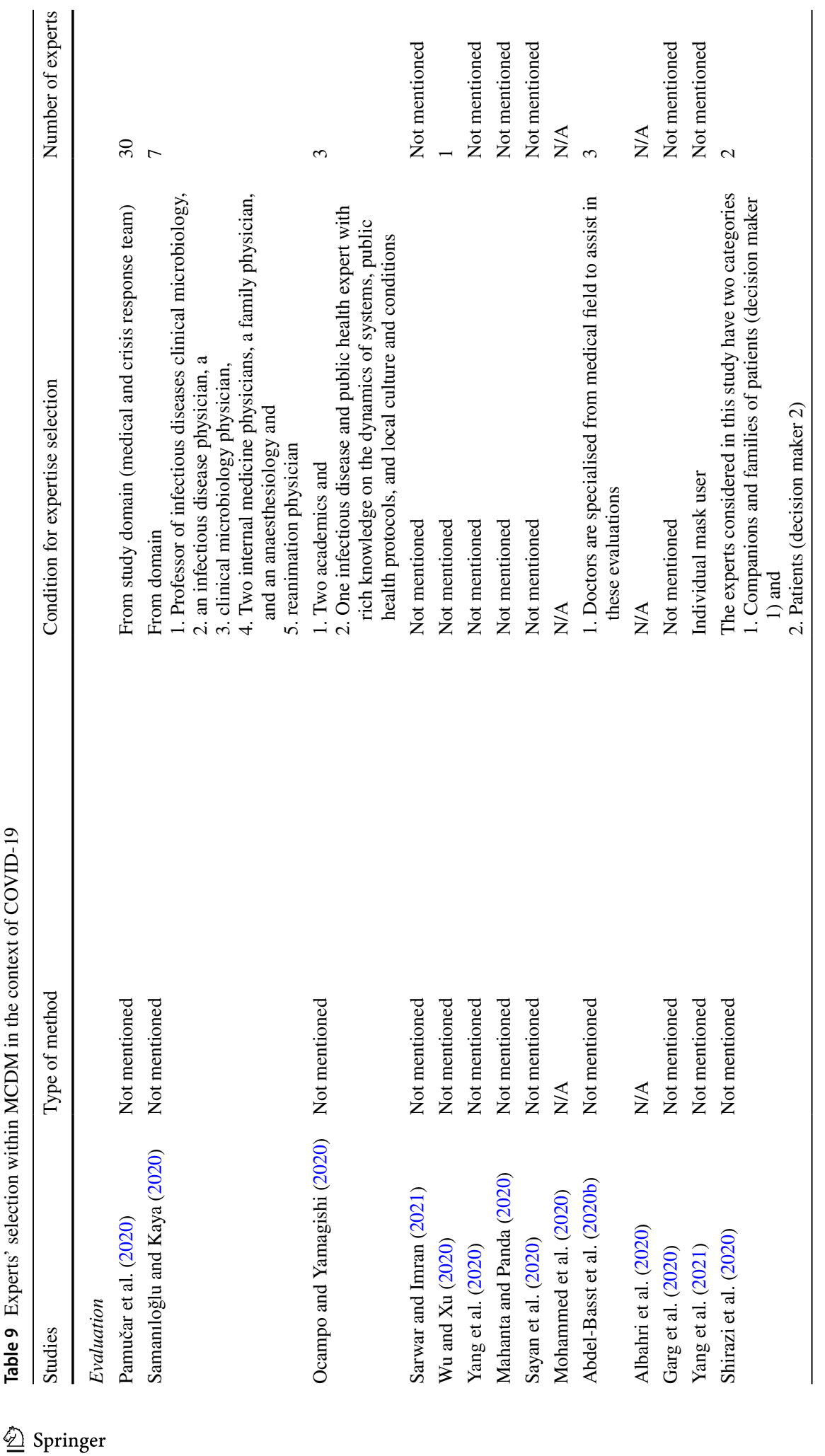




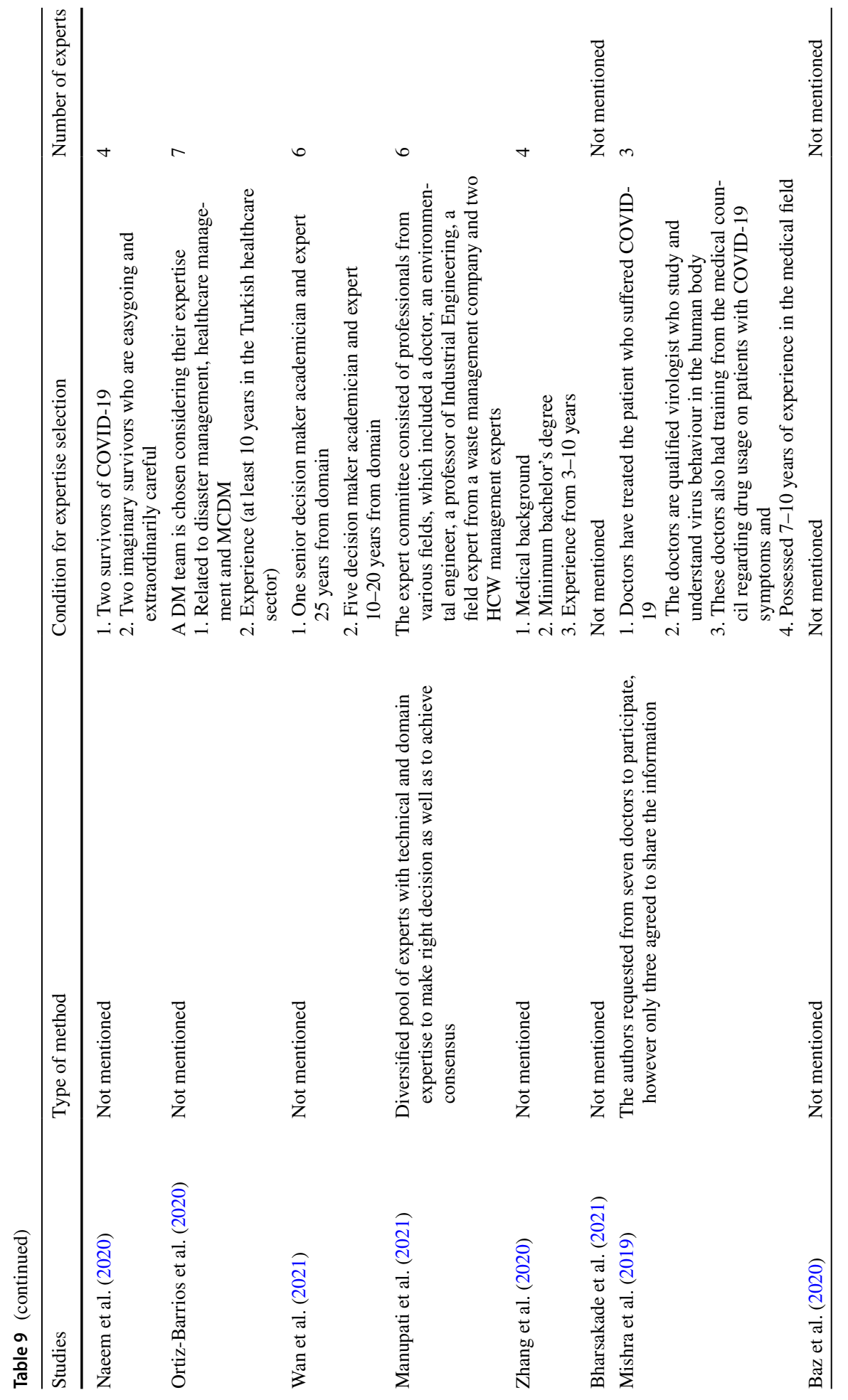




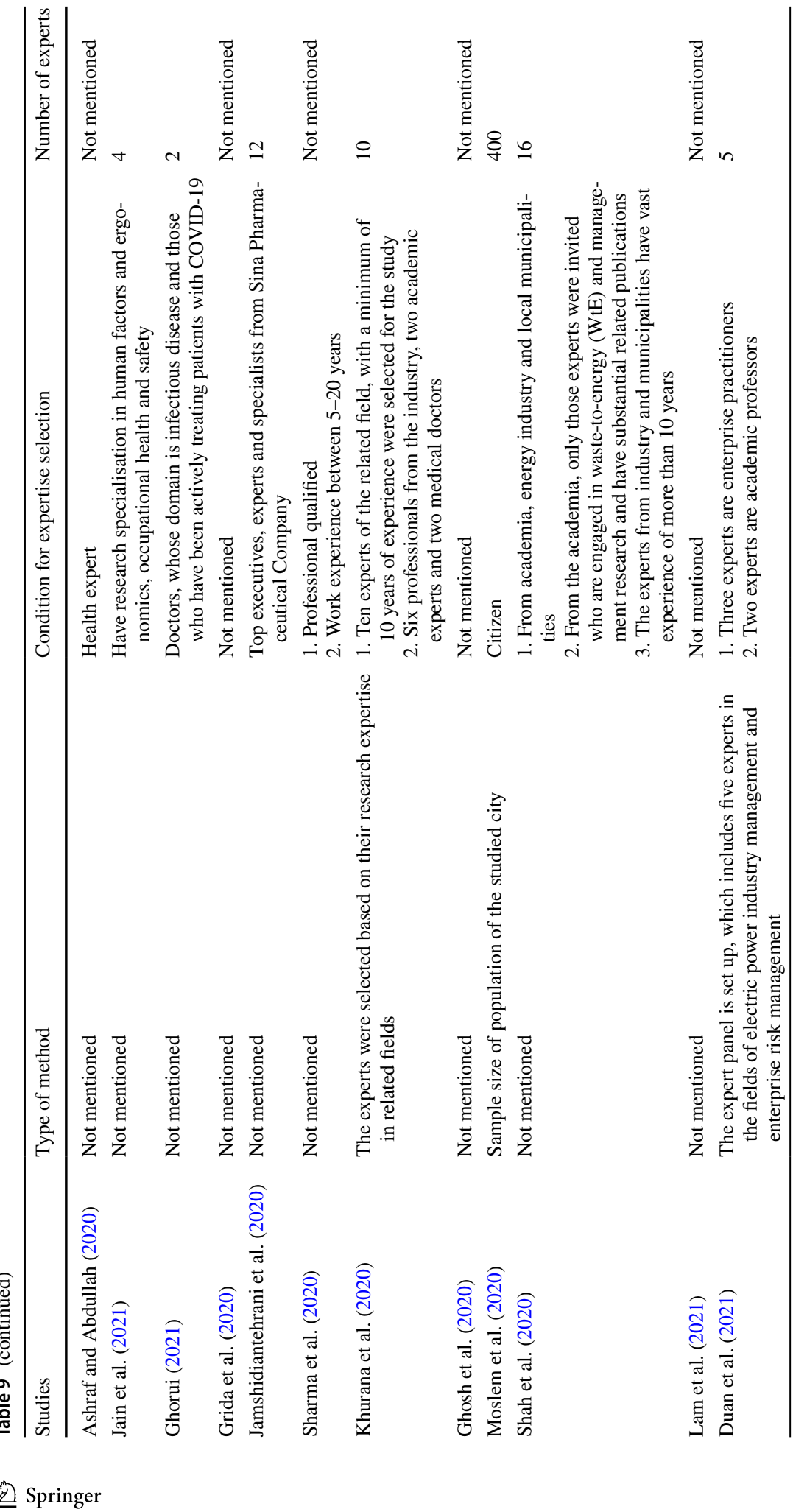




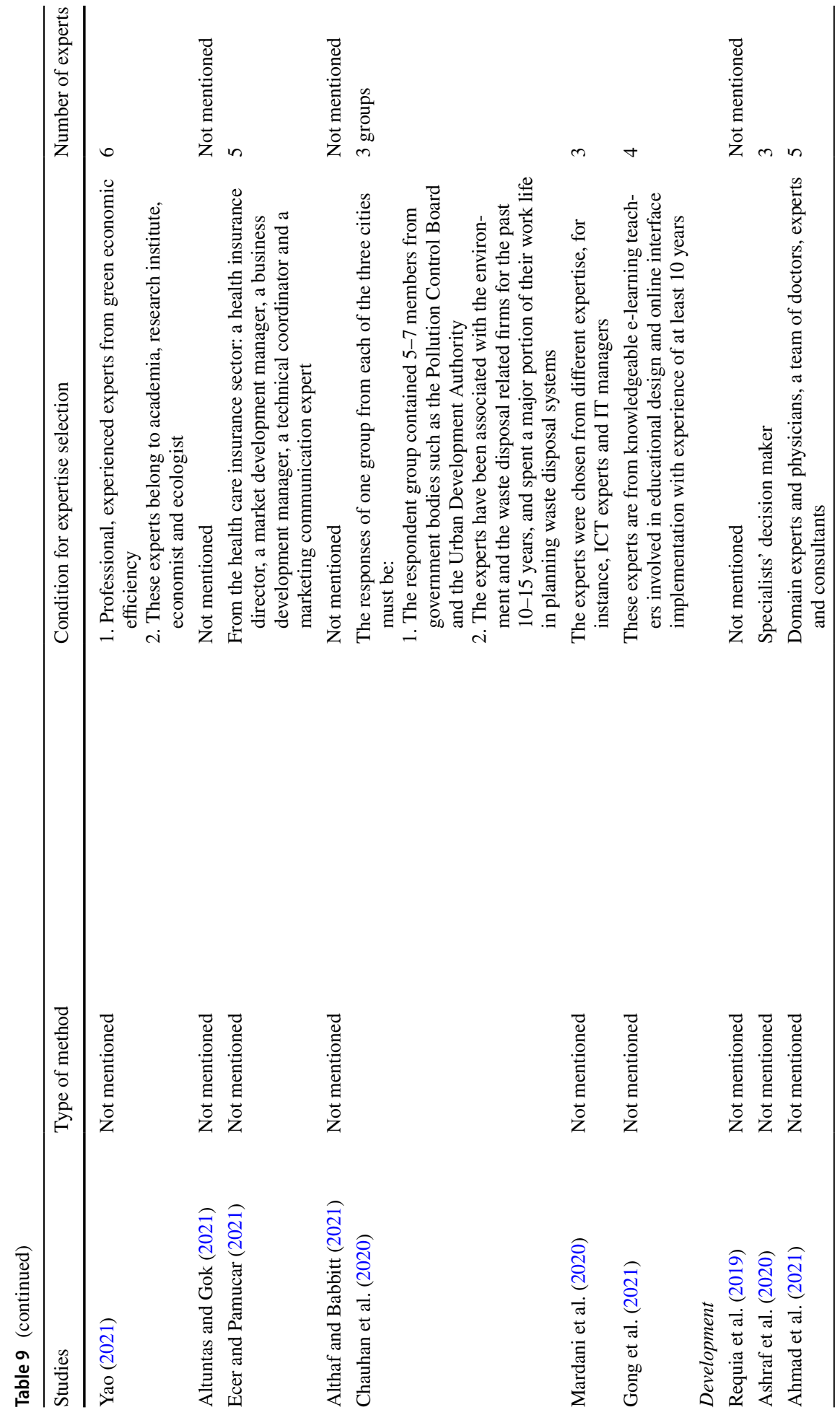




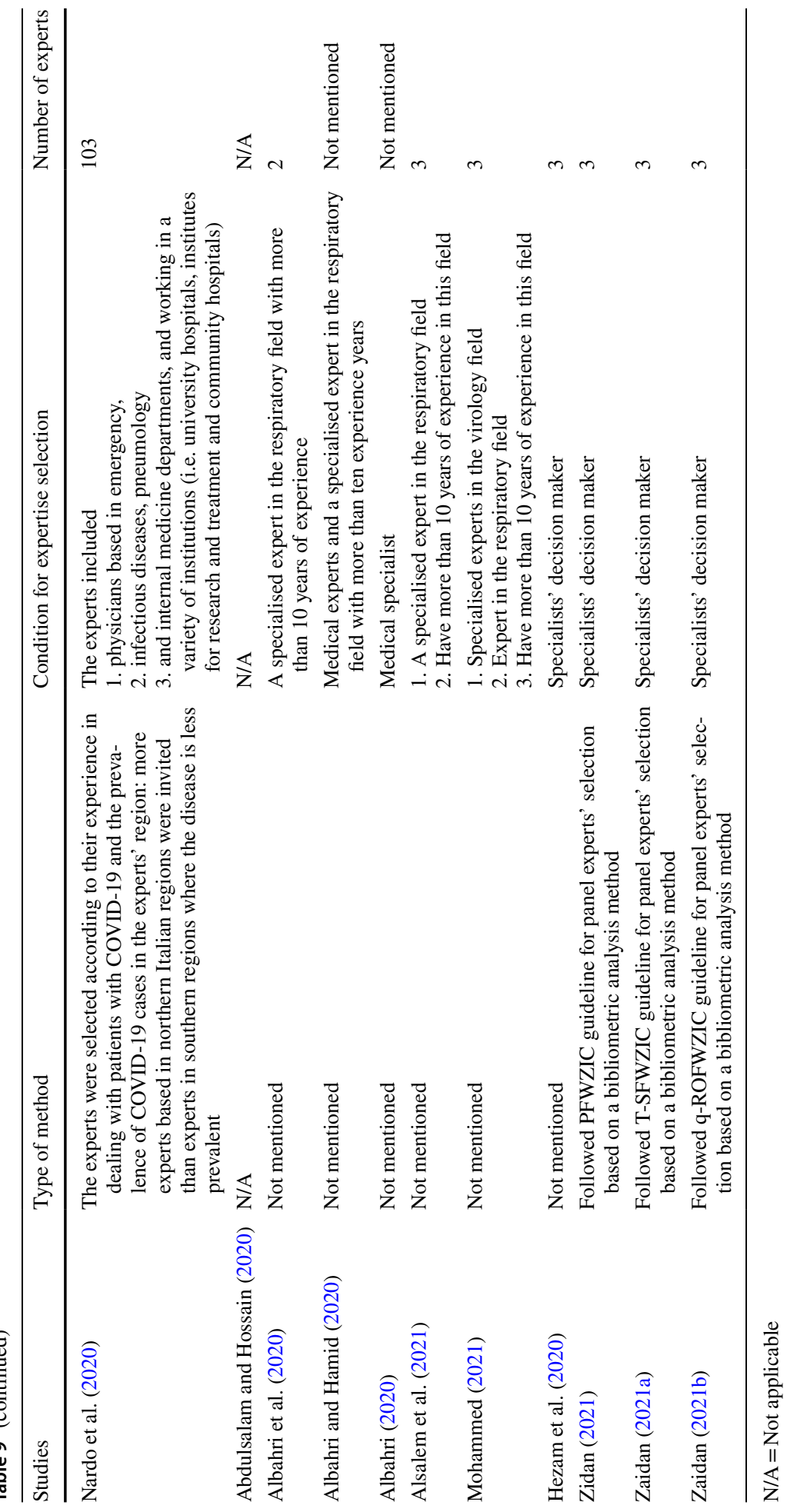


making results. The values of the operator's parameters (p, q) within a specific interval are tweaked accordingly to observe its impact. This sensitivity can indicate and evaluate the validity and stability of the ranking results in the proposed methodology compared with other MCDM methodologies by competitors (Zidan 2021) and systematic ranking evaluation using a statistical approach. This statistical analysis is applied to validate the ranking results of the proposed MCDM framework. In this approach, the rank results of the alternatives are divided into (n) groups. Each group consists of a set of the studied alternatives. The mean \pm standard deviation computes for each group. For example, suppose we divided the rank results of the alternatives into three groups; according to this division, the best value should be for the first group, which must be supported by the mean \pm standard deviation result. The statistical measurement of all following groups should be poorer than the first and preceding groups, while such measurement should be better or equal to the posterior groups, respectively, to ensure that the alternative prioritisation undergoes systematic ranking(Alsalem et al. 2021). However, limited research work assessed the MCDM methods and examined and compares its validity under different situations and parameter settings. In (Wu and Tiao 2018; Ghaleb et al. 2020), a new validation scheme was proposed to test the pertinence and effectiveness of the MCDM methods based of defined set of parameters (i.e. computational complexity refers to the intricacy of time of the MCDM method process, number of alternative processes and criteria, adequacy in supporting group decision making refers to the flexibility of the MCDM method in acquiring the consensus of decision maker information, addition or removal of criteria refereeing to which extend that the procedure of the tested MCDM method ease in adding or removing new criterion, and different decision matrices refer to assessment of the MCDM method with different number of criteria and alternatives) for statistical assessment of the appropriateness of the basic MCDM methods with different parameter setting and conditions. Table 10 presents the MCDM methods that were employed in the context of COVID-19 and underwent validity assessment based on the parameters.

According to the literature (Wu and Tiao 2018; Ghaleb et al. 2020; Ilyina et al. 2019), few classical MCDM methods underwent validity schema assessment, namely, PLP, AHP, TOPSIS, VIKOR and ELECTRE II. Three of these classical MCDM methods in their original or extension versions were utilised to cope with the DM problem in the context to COVID-19 for both perspectives (i.e. evaluation and development), as presented in Table 10. In the validity schema, five different parameters were used to test and examine MCDM methods' performance. This assessment showed that the AHP approach was the most effected method when parameter settings (i.e. number of alternatives, number of criteria, expert group) were changed, whereas TOPSIS recorded the highest computational complexity. Noticeably, the VIKOR method showed the lowest effect by parameter changing and less computational complexity (Ghaleb et al. 2020). In summary, out of 41 studies under the evaluation category, $(26,63.41 \%)$ studies employed these three MCDM methods in their evaluation framework, $(11,42.3 \%)$ used the AHP method or AHP extension versions (Samanıloğlu and Kaya 2020; Albahri et al. 2020; Sarwar and Imran 2021; Shirazi et al. 2020; Ortiz-Barrios et al. 2020; Bharsakade et al. 2021; Baz et al. 2020; Ghorui 2021; Khurana et al. 2020; Ghosh et al. 2020; Yao 2021)), (9, 34.61\%) used TOPSIS or TOPSIS extension versions ((Sayan et al. 2020; Mohammed et al. 2020; Abdel-Basst et al. 2020b; Naeem et al. 2020; Ortiz-Barrios et al. 2020; Ashraf and Abdullah 2020; Ghorui 2021; Grida et al. 2020; Althaf and Babbitt 2021)) and (6; 23.07\%) used VIKOR or VIKOR extension versions (Albahri et al. 2020; Wan et al. 2021; Jain et al. 2021; Shah et al. 2020; Lam et al. 2021; Yao 2021). In the development category, out of 14 studies, (11, 78.57\%) adapted these three classical methods or their extension. The highest group of studies was 


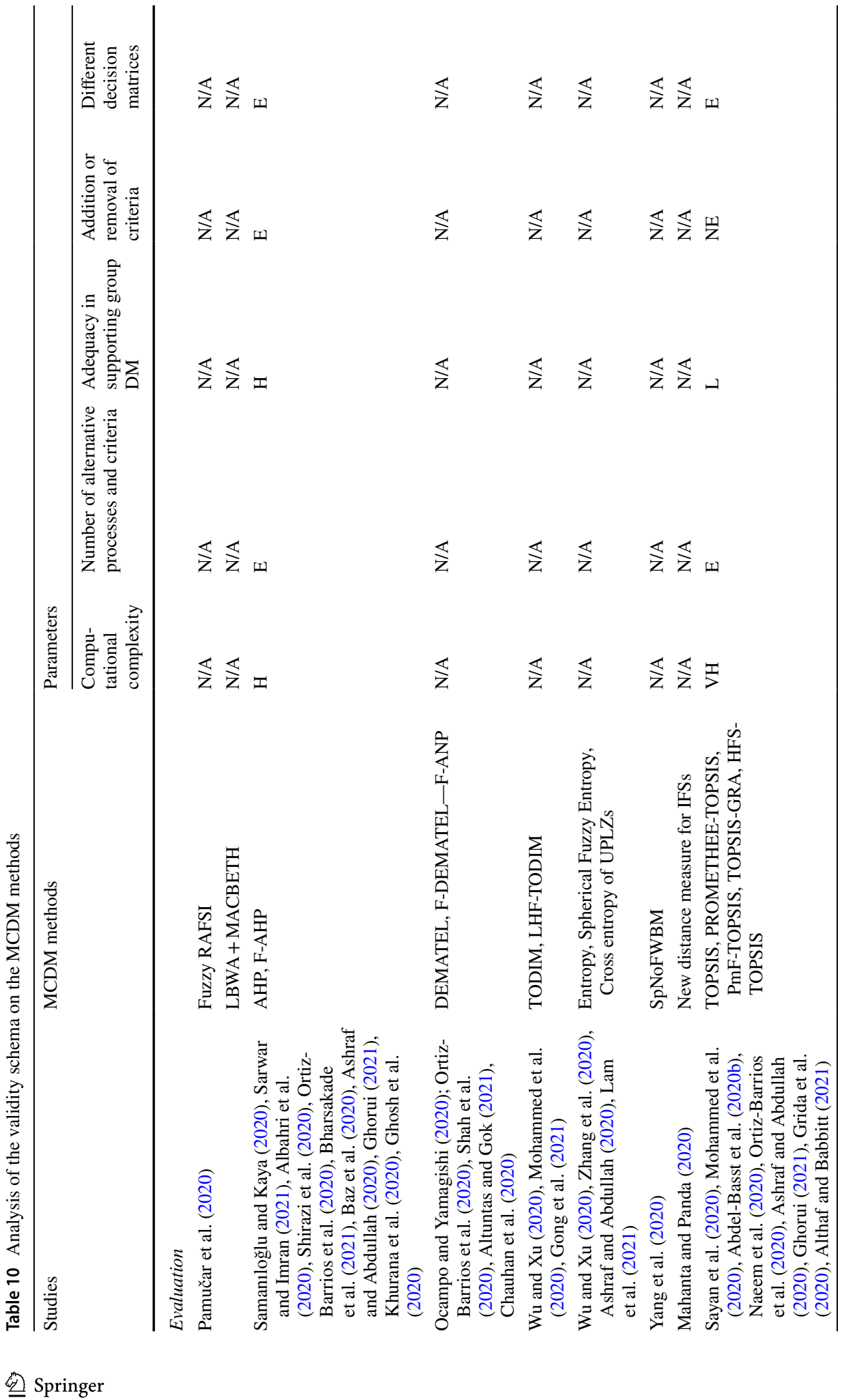




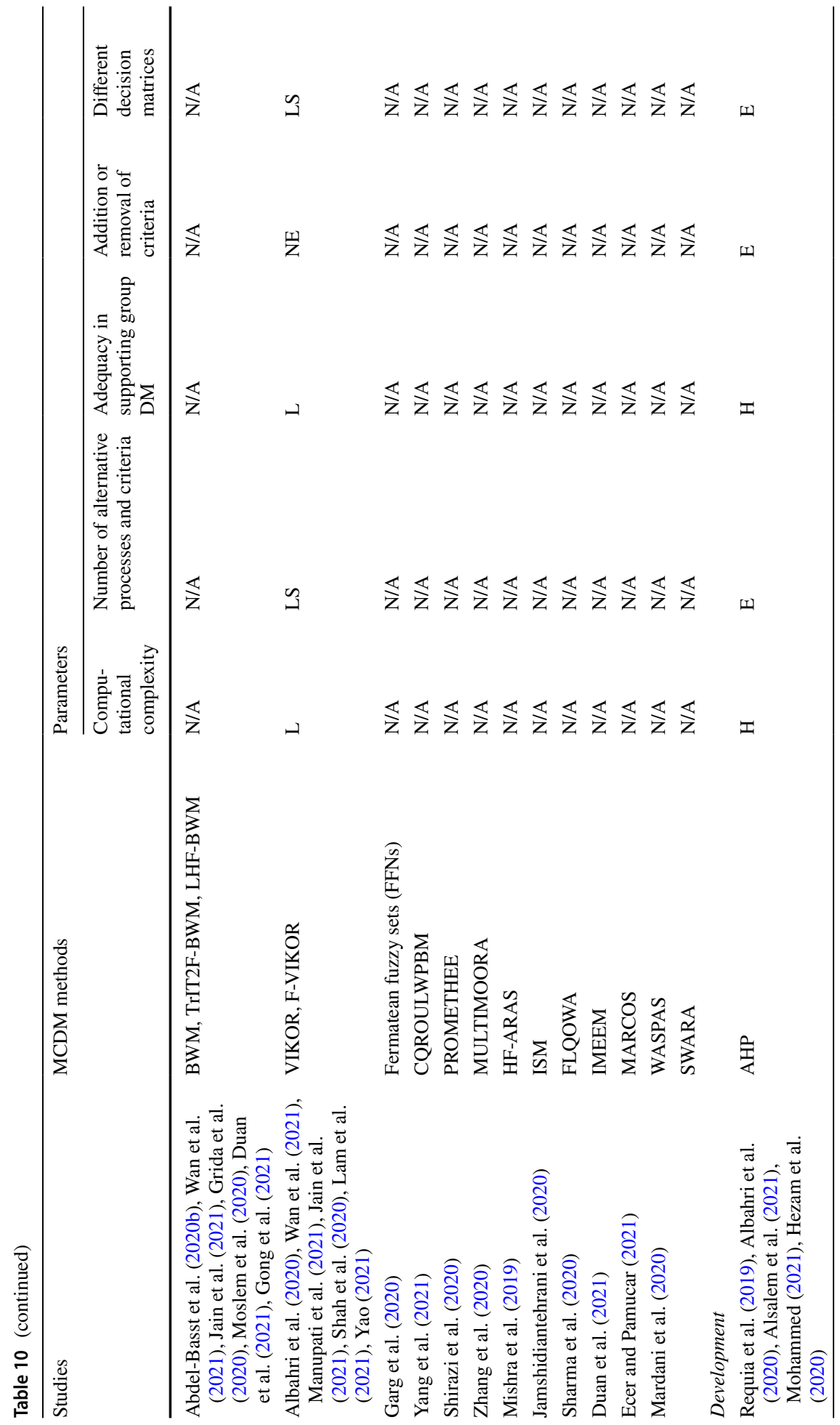




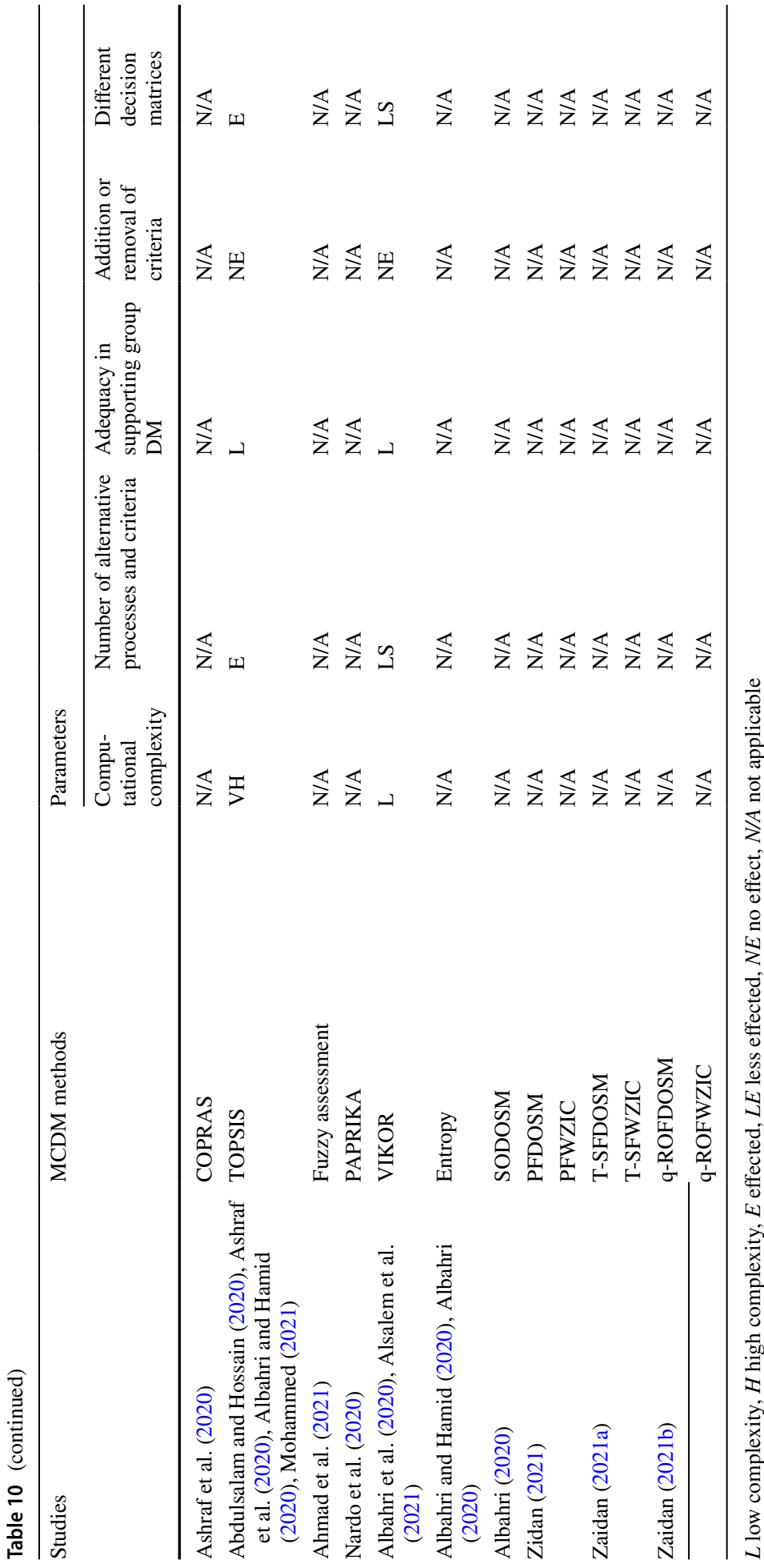


under AHP (Requia et al. 2019; Albahri et al. 2020; Alsalem et al. 2021; Mohammed 2021; Hezam et al. 2020), followed by TOPSIS (Abdulsalam and Hossain 2020; Albahri and Hamid 2020; Mohammed 2021) and VIKOR (Albahri et al. 2020; Alsalem et al. 2021) methods or their extension with $(5,45.45 \%),(3,27.27 \%)$ and $(2,18.18 \%)$, respectively. The MCDM methods are continuously proposing new or extension methods, and each of them requires different levels of computational intensity and relies on the different parameter settings based on the application area of the DM problems that may produce different outputs. Consequently, they emphasise that the importance of investigating the suitability of the DM method to rank the decisions in consideration of the DM conditions is important in determining the quality of the recommended decision and the effort required to obtain it. However, discussing all of MCDM methods and examining their validation in all possible situations is nearly impractical. Thus, the proposed validation scheme by $\mathrm{Wu}$ and Tiao (2018); Ghaleb et al. 2020) can serve as a guideline for assessing the validity and appropriateness of the unexamined methods other than comparing with exciting methods. Moreover, other parameter settings such as aggregation settings and operators, normalisation of approaches and effectiveness of fuzzy environments need to be included in the validation schema for a comprehensive assessment of the MCDM methods (Wu and Tiao 2018; Ghaleb et al. 2020; Zamani-Sabzi et al. 2016).

\subsubsection{MCDM theoretical challenges}

Many theoretical challenges have been addressed within the MCDM method, and they are analysed in this conducted study following the MCDM method types The frequencies of said challenges are presented in Table 11. In the base of weighting method theory, five different challenges were mentioned in literature of the COVID-19 context, The first four related to the subjective and hybrid weighting approaches, while the dynamic change related to the objective weighting approach, as follow:

- Unnatural comparison: a process of comparing two different criteria based on comparing scale (i.e., 1 to 9) to indicate how many times this specific criterion is better than other criteria is an unnatural comparison process required from decision maker/s additional cognitive ability to deal with such issue. Thus, for making better decisions, the comparison must be based on similar quantities and be a natural process (Albahri 2020).

- Inconsistency: is the most common theoretical challenge of the subjective weighting methods that depend on pairwise or reference comparison amongst criteria in determining the important weight of criteria. This inconsistency issue appears more clearly when a number of criteria are high (Moslem et al. 2020).

- Time consumption in a pairwise comparison: It demonstrates that conducting a comparison is time-consuming and often necessitates sufficient explanation to the person conducting the comparison. (Abdel-Basst et al. 2020b)

- Global the weight (aggregating the weights): is a challenge that occurs when the weight of criteria is needed and there's $n$ of decision-makers involved and conducting the process of weighting criteria must implement $\mathrm{n}$ times and there will be $\mathrm{n}$ weight values for each criterion need to aggregate and find the decision-makers consensus. (Duan et al. 2021) 


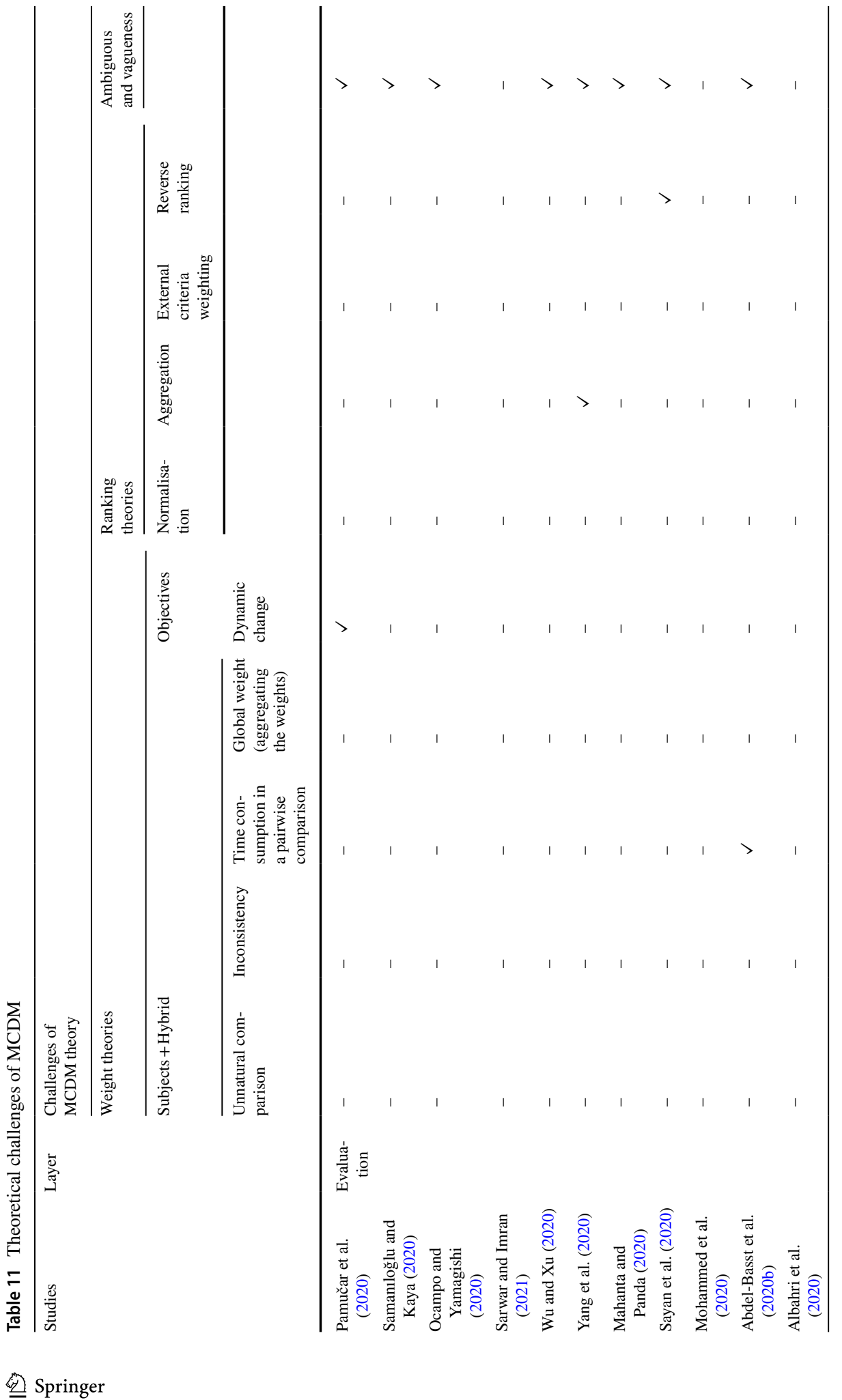




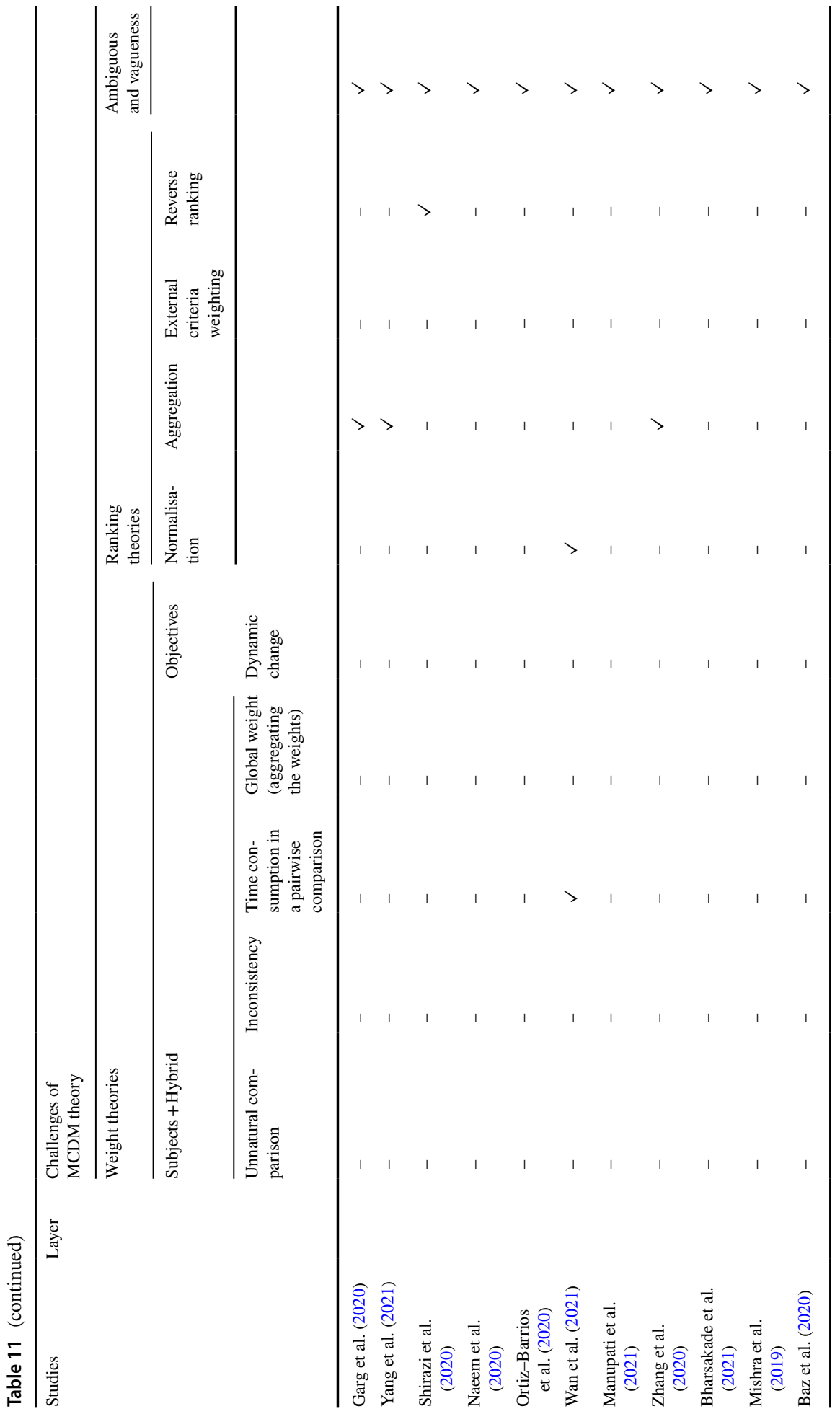




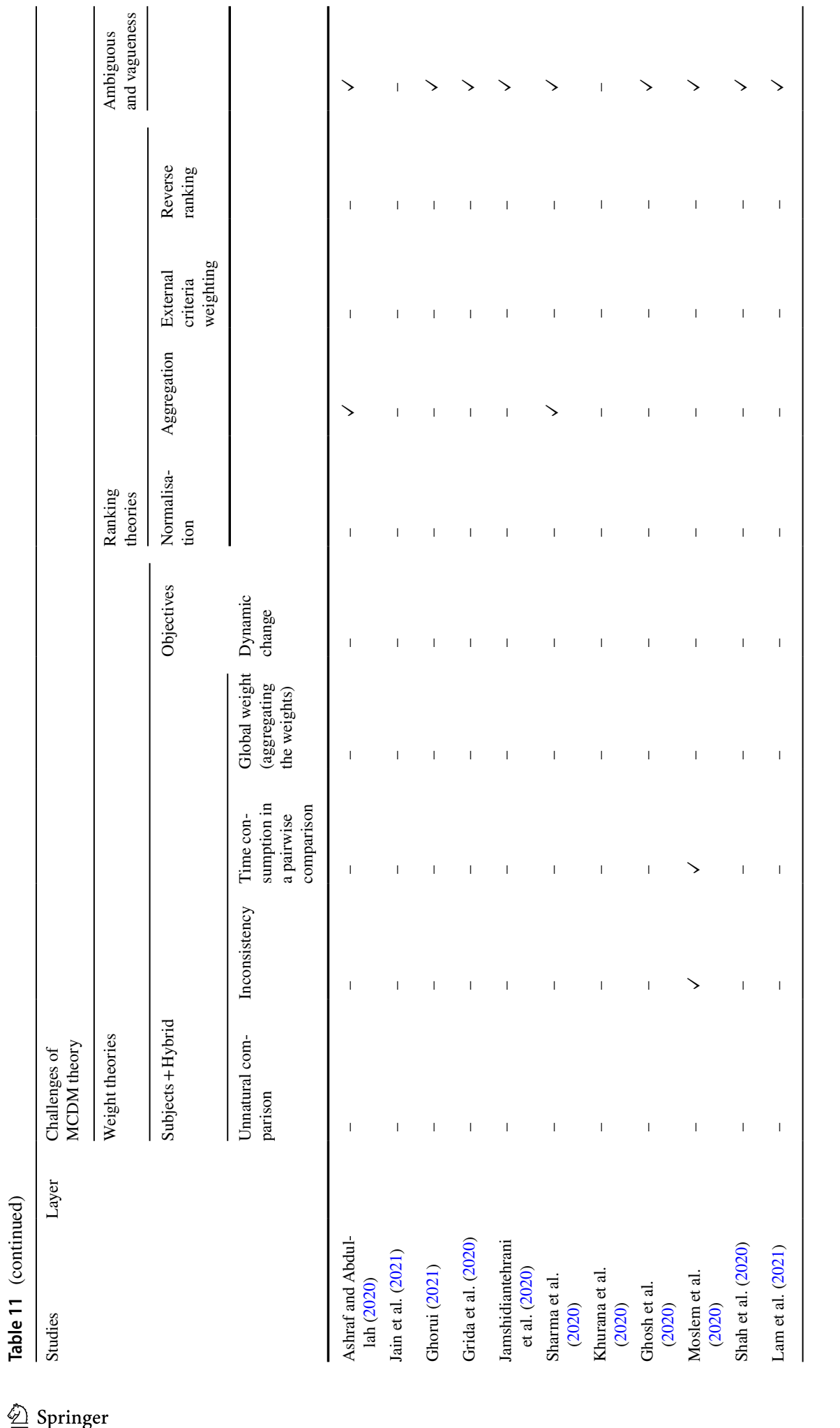




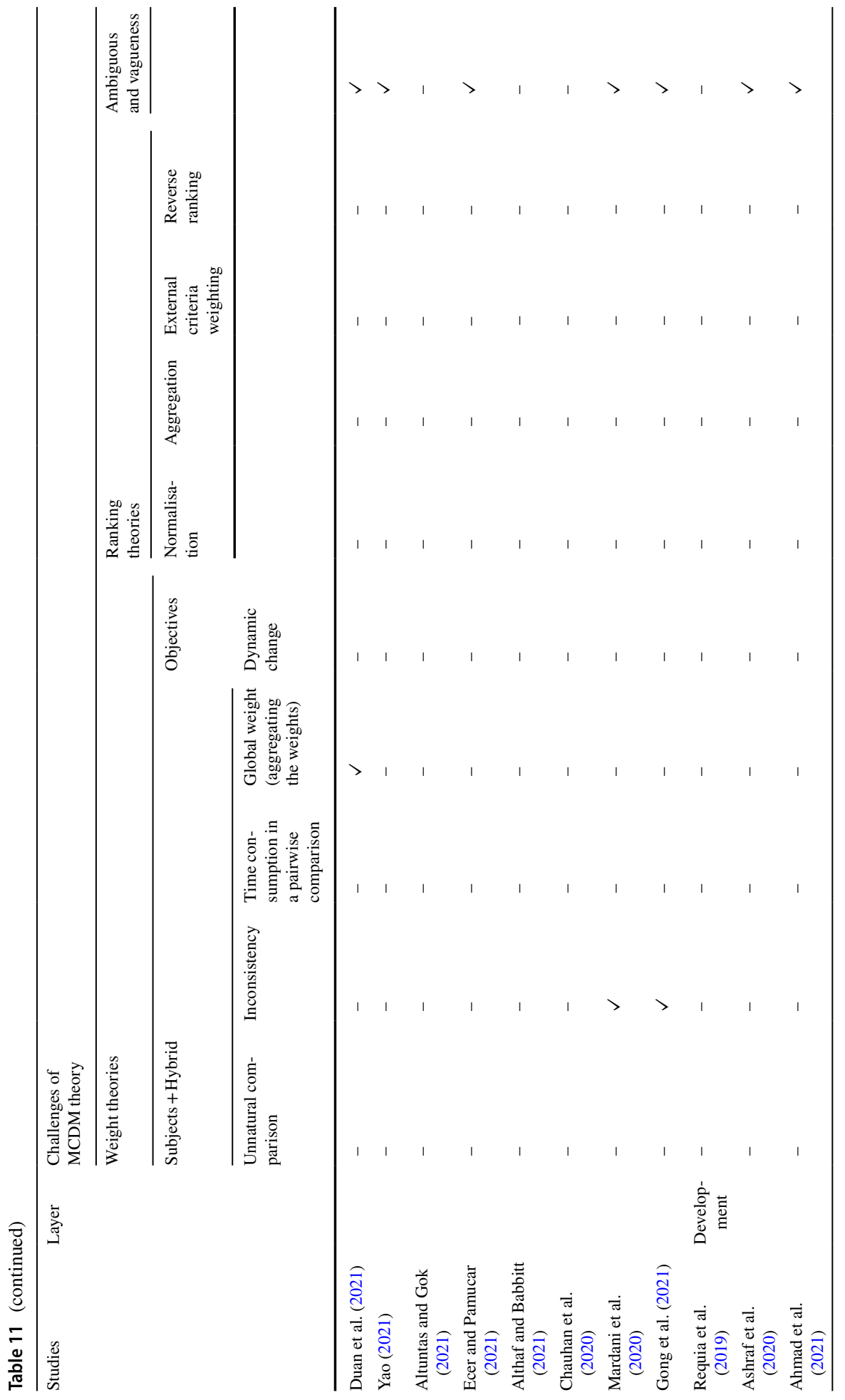




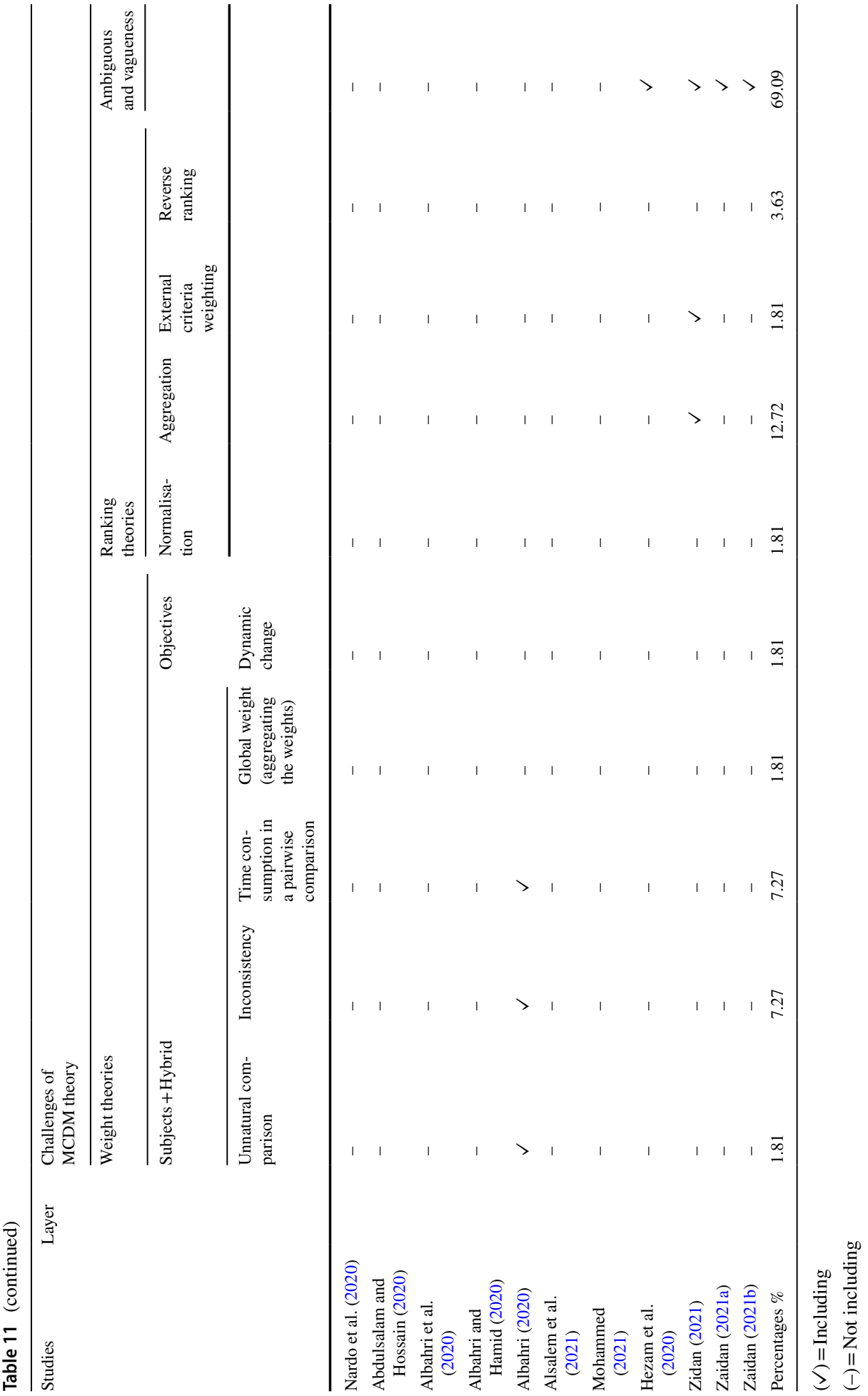


- Dynamic change: an issue in objective weight methods which rely on the raw data in computing the criteria weight. Thus, any change in the dataset, which very commonly happening, will affect the importance level of the criteria (Pamučar et al. 2020)

Four main challenges are related to ranking method theory which are:

- Normalization: It is necessary to unify diverse evaluation scales, and their values must be transformed into unitless numbers; however, using different strategies for normalisation may result in varied ranking/weighting results (Wan et al. 2021)

- Aggregation: mostly, all MCDM problems rely on aggregation functions to aggregate the performance of alternatives against multiple criteria. Hence, the utilized aggregation function may have a direct impact on the decision-making results (Ashraf and Abdullah 2020)

- External Criteria Weighting: a theoretical challenge and limitation in ranking methods that required to compute the criteria weight externally and aggregate it to the decision matrix to find the final rank, (Zidan 2021)

- Reverse ranking: The issue of rank preservation and reversal is crucial in ensuring the effectiveness of MCDM procedures, which is considered a critical factor in achieving trustful and stable results. In this issue, when an alternative is added to or removed from the list of the existing alternatives, the order in which the alternatives are prioritized is inverted (Shirazi et al. 2020)

- Ambiguous and vagueness challenge: MCDM procedures incorporate decision makers' preferences and subjective judgments, including quantitative and/or qualitative criteria ratings. Which might be vague, uncertain, and ambiguous, complicating the decisionmaking process when applied in real-world problems (Samanıloğlu and Kaya 2020).

To provide a clear discussion for the challenges presented in Table 11, both categories were analysed together (evaluation and development) concerning weight and ranking theories' challenges.

For the weight theories challenges, four challenges were outlined with respect to subjective weight and hybrid weight (hybrid weight includes subjective and objective weights). The distribution percentage results for these four challenges can be summarised as follows. For unnatural comparison that obtained $(1.81 \%)$, the splitting of the problem into smaller, more tractable sub problems can provide the reasonable solution, but unnatural pairwise comparisons did not enable the analyst to estimate how irrational the judgments of given experts are.

The role of inconsistency challenges indices is precisely that of quantifying the deviation of a set of pairwise comparisons from being consistent and obtained (7.27\%). Time consumption in a pairwise comparison was highlighted and obtained (7.27\%). The traditional pairwise comparison applicable for smaller sample size cannot exceed larger pairwise comparison size, and this leads to time consumption. Determining aggregate numerical criteria weights (global weight) from a group of multiple decision makers provides another challenge and obtained (1.81\%). The view of dynamic criteria was clarified in the fifth challenge (dynamic change 1.81\%) that focused on objectives' weight. In general, dynamic criteria are conceptualised as changes in group average performance over time, changes in validity over time and changes in the ranking of scores on the criterion over time.

For the ranking theories challenges, 8 studies (12.72\%) were dropped in the aggregation challenges, but aggregation can generate problems of its own. Not only does it lead to a 
loss of COVID-19 information but it also can leave analyses vulnerable to the 'ecological fallacy' when ranking different alternative cases and dealing with such infectious disease data. On the one hand, normalisation is an essential step in data analysis and for MCDM methods where different normalisation techniques that can be used in MCDM problems, as mentioned in Table 7, to reduce complexity overall and can improve ranking alternatives. Clearly, only one study (Wan et al. 2021) (1.81\%) was within the evaluation category. The numerous normalisation methods provided in the literature could eliminate this issue; thus, minimal attention was given to overcome this challenge. For the reverse ranking challenge that obtained $(3.36 \%)$, overcoming such issue can effectively reduce the high degree of uncertainty. Finally, for external criteria weighting, one study $(1.81 \%)$ reported this challenge, where different MCDM methods used external weight to provide the final rank for the alternatives. However, one of the main limitations in the MCDM method is the need for external weighting methods for the final decision of the best alternative. The high percentages challenges were located under the ranking theories challenges, especially for ambiguous and vagueness type (69.09\%), whereas minimal attention was highlighted for other types. However, inconsistency and time consumption in pairwise comparison challenges play a key role not only for the weight theories but also can highly affect ranking theories and need an accurate design solution.

\section{Future pathway}

This section discusses several of the most considerable future pathway directions for medical research, including COVID-19 with MCDM. After comprehensive literature discussion in this review, those future pathways became clear.

Firstly, with the concern of scattered criteria used in various cases of research, as discussed in Sect. 2.4, the defined set of criteria and sub criteria were mainly collected from literature but not unified. Consequently, each study, even those that discussed the same topic, depended on different sets of criteria that undoubtedly affected the accuracy of the final decision. Hence, standardising the multicriteria set is a necessity. The Fuzzy Delphi Method (FDM) is a proven effective method in unifying, screening and forecasting, assessment, standardisation and criteria identification in various domains (Rahimianzarif and Moradi 2018; Pham et al. 2017; Manakandan et al. 2017; Morovati Sharifabadi et al. 2016; Sultana et al. 2015; Kamarulzaman et al. 2015; Abdulkareem et al. 2021). It is mainly used to provide appropriate results when making decisions regarding objective issues needed whilst the involved criteria are not unified (Abdulkareem et al. 2021; Dawood et al. 2021). The FDM provides a resilient framework that can handle the lack of precision and clarity. The incomplete or inaccurate information is considered an issue in decision making. Furthermore, subjectivity in the decisions made by the decision makers cause uncertain results. The FDM is tailored to the fuzzy environment to handle imprecise descriptions and human subjectivity (Rahimianzarif and Moradi 2018; Kamarulzaman et al. 2015). Therefore, it is the best method for assessing and unifying the most effective criteria with high flexibility scale (Rahimianzarif and Moradi 2018; Manakandan et al. 2017; Abdulkareem et al. 2021) in the context on COVID-19. Moreover, all vital information are considered without any missing because the membership degree effectively considers all experts' preferences (Manakandan et al. 2017; Dawood et al. 2021; Wu et al. 2020). 


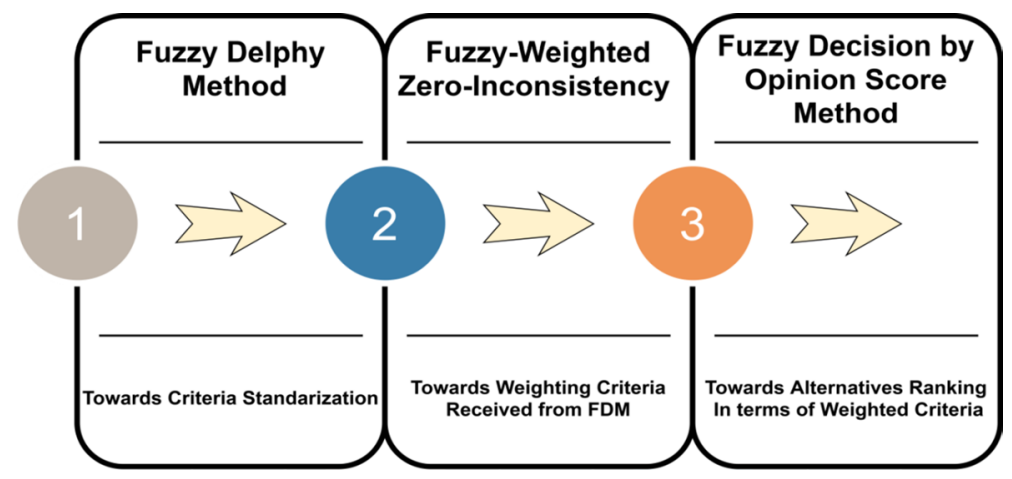

Fig. 8 Pathways and future direction phases

Secondly, the process of ranking or prioritising alternatives based on the standardised criteria is needed. Different MCDM methods for addressing various MCDM problems in the context of COVID-19 were presented in the literature, as mentioned in Sect. 2.5. However, a method called FDOSM and its extensions developed by Zidan (2021); Zaidan 2021a; Zaidan 2021b; Salih et al. 2020a) presented a unique solution for addressing the issues associated with previous techniques by considering the concept of an ideal solution, reduced the number of comparisons, defined fair and implicit understandable comparisons, prevented inconsistency, reduced vagueness and yielded a minimum number of mathematical operations. Utilising such method in the content of MCDM with various COVID-19 case studies is warranted. FDOSM weighting for the criteria values of each alternative is implicit; it is limited to calculating the weight for each criterion implicitly, which is considered a theoretical issue (Salih et al. 2020b).

Lastly, to resolve this issue, the criteria must be determined for their importance, which is measured by weight in the context of MCDM. Many weighting criteria methods have been used in the literature such as BWM (Abdel-Basst et al. 2020b), AHP (Samanıloğlu and Kaya 2020; Albahri et al. 2020) and FWZIC method with its extension (Zidan 2021; Zaidan 2021a, 2021b; Mohammed et al. 2021). Noticeably, amongst all MCDM weighting methods, FWZIC either in its original version or extensions was proven in providing weights for criteria with zero inconstancy, and it was more robust in mitigating the theoretical challenges of the previous weighting techniques (i.e. subjective, objective or hybrid weighting methods) discussed in Sect. 2.11 including the number and nature of the comparison, time consuming and inconsistency, in addition to the effect of raw data change. Hence, employing FWZIC in computing the criteria weight has promising results in terms of the accuracy and the consistency of the final decision. Consequently, future pathway solution is meant to address these issues. Therefore, certain phases are proposed, as shown in Fig. 8. Each of these pathways is presented in detail in the following sub sections.

\subsection{Fuzzy Delphi method}

This method is derived from the traditional Delphi method along with fuzzy theory to tackle the issues of typical DM shortcomings and improve precision (Murray et al. 1985). Research works towards advancing this method have been continuous such as fuzzy integration algorithms (Ishikawa et al. 1993). The improvement to the FDM was implemented 


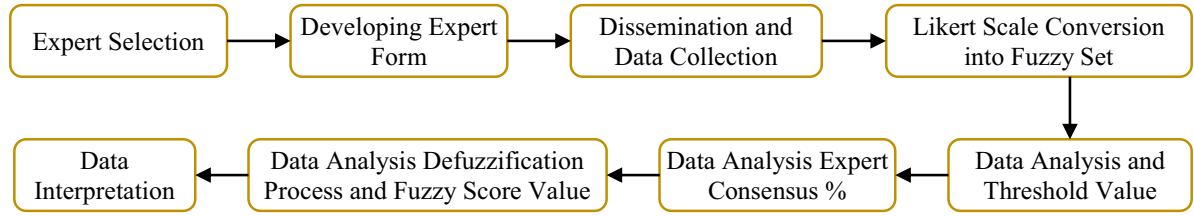

Fig. 9 Fuzzy Delphi Method

Table 12 Linguistic variables for five scales

\begin{tabular}{lll}
\hline Likert scale & Linguistic change enable & Fuzzy scale \\
\hline 1 & Strongly Disagree & $(0.0,0.0,0.2)$ \\
2 & Disagree & $(0.0,0.2,0.4)$ \\
3 & Less Agree & $(0.2,0.4,0.6)$ \\
4 & Agree & $(0.4,0.6,0.8)$ \\
5 & Strongly Agree & $(0.6,0.8,1.0)$ \\
\hline
\end{tabular}

to reflect weight differences in relation with expert's ability (Garai 2013). In FDM, it functions either by using fuzzy statistics or by continuous mathematical explicit membership functions to steady the iteration (Chang et al. 2011). Although this approach is useful in unifying criteria and establishing importance level for them, its main steps are warranted to be recognised, especially whilst consider applying it for future MCDM and Covid-19 research. FDM has eight steps, as presented in Fig. 9.

First Step Experts are selected for appropriately obtaining their opinions on the criteria. This stage requires a careful selection, where each expert involved must have adequate, related opinion with the case study identified. Aside from selecting an expert in itself, the following issues on the adequate number of experts suitable for the process arise. FDM in its basic nature considers all expert opinions based on their consensus; thus, expert numbers in this regard matter. Different works calls for dissimilar number of experts to be utilised whilst working with FDM theory (Adler and Ziglio 1996). The author discusses that a number of experts is sufficient and acceptable only when uniformity amongst them is very high.

Second Step A questionnaire that carries all the features and criteria for the standardisation is developed for experts. Two common approaches in developing such questionnaire rely on criteria identified from literature review or by interviews. According to Powell (2003), FDM questionnaire is conducted in the first round to address the issues or any misconception experts might have. Open-ended questions can be adopted or even used from previous studies (Duffield 1993).

Third Step This step includes data dissemination and collection from the expert chosen in previous steps. The process is either done by directly meeting each expert or by making an online version using email.

Fourth Step This step begins by converting all the data collected from experts into triangular fuzzy numbers using the scale presented in Table 12.

Fifth Step Data analysis is conducted based on the triangular fuzzy numbers towards acquiring the threshold value. The threshold value must be equal to or less than 0.2 because it presents first acceptance conditions (Cheng and Lin 2002). The distance between two fuzzy numbers $m=\left(m_{1}, m_{2}, m_{3}\right)$ and $n=\left(n_{1}, n_{2}, n_{3}\right)$ can be computed using the vertex method: 


$$
d(\tilde{m}, \bar{n})=\sqrt{\frac{1}{3}\left[\left(m_{1}-n_{1}\right)^{2}+\left(m_{2}-n_{2}\right)^{2}+\left(m_{3}-n_{3}\right)^{2}\right]} .
$$

Sixth Step Expert consensus is calculated, and it must be greater than $75 \%$ in accordance with the second condition to be accepted (Chu and Hwang 2008).

Seventh Step This step includes defuzzification to acquire the fuzzy score. The value for ' $A$ ' must be more than or equal to the medial alpha level of 0.5 to fulfil the approval for the last condition (Bodjanova 2006). Furthermore, ' $A$ ' can be further utilised in identifying the priority element based on onions. Fuzzy score ' $A$ ' can be implemented with the use of the following equation:

$$
A=\left(\frac{1}{3}\right) X\left(m_{1}+m_{2}+m_{3}\right)
$$

Eighth Step This step is fuzzy FDM data interpretation, where all data interpretation for FDM is described based on the acceptance and rejection rules. Three requirements are needed for an item to be discarded or kept based on the consensus of experts as following.

First rule The expert agreement is individually evaluated for the conducted item in accordance with the triangular fuzzy numbers. A threshold of less than or equal to 0.2 must be accomplished for an item to be accepted (Cheng and Lin 2002).

Second rule For an item to be considered, the majority of all threshold must be greater than or equal to $75 \%$ (Chu and Hwang 2008).

Third rule The level of importance for a factor within an item can be defined using pseudo partition that is established by consulting an expert by setting threshold to prevent bad partitioning (Bodjanova 2006).

\subsection{Methodology of fuzzy-weighted zero inconsistency}

The fuzzy-weighted zero inconsistency (FWZIC) method is proposed to compute the importance of criteria with zero inconsistency. This method came in four different versions with different fuzzy types. FWZIC requires six phases until completion. The first phases are identical despite the type of fuzzy environment utilised, but the latest three steps require different mathematical operations depending on the fuzzy environment. All the similar phases are discussed between versions, and the differences are highlighted in the table along with their difference starting with Phase 4 until Phase 6.

\subsubsection{Phase 1: Criteria definition}

Upon the completion of FDM, different sets of criteria are passed to FWZIC. In the first step, these criteria are evaluated and defined. The following step includes classifying and categorising all the criteria along with their sub criteria.

\subsubsection{Phase 2: Structured expert judgment}

Structured expert judgment (SEJ) includes evaluating all the defined criteria from the previous step for their importance level. The evaluation is performed by panel of experts with experience on the study area of the case study for the criteria. Upon the completion of expert list, nomination is performed in accordance with the following steps. 
Table 13 Five-point Likert scale and equivalent numerical scale

\begin{tabular}{ll}
\hline Numerical scoring scale & Linguistic scoring scale \\
\hline 1 & Not important \\
2 & Slight important \\
3 & Moderately important \\
4 & Important \\
5 & Very important \\
\hline
\end{tabular}

3.2.2.1 Step 1: Expert identification The expert in the FWZIC context refers to a person who was or is still involved in the subjects of the case study and regarded as knowledgeable by others. Experts in the literature are also recognised as 'domain' or 'substantive' experts.

3.2.2.2 Step 2: Expert selection Upon the completion of expert identification, a set of experts is selected for the case study. At least four experts are needed in this stage. All experts from the previous stage are contacted by email to determine their availability and willingness to be considered as potential expert for the panel.

3.2.2.3 Step 3: Evaluation form development The evaluation form is done owing to its importance as important tool for collecting expert consensus. Before finalisation, it is checked for reliability and validity, and reviewed by all the experts from the previous step.

3.2.2.4 Step 4: Defining the importance level scale All the experts selected from the previous step define the importance level for each criterion with the use of a 1-5 Likert scale.

3.2.2.5 Step 5: Converting linguistic scale into numerical scale All preference values are transformed from subjective form into numerical form for utilisation in the analysis. Thus, the importance level for every criterion from each expert in the used Likert scale is converted into numerical scale, as shown in Table 13.

\subsubsection{Phase 3: Building the EDM}

The EDM with the main parts, which include criteria and the alternatives, is created, as shown in the following table.

The previous phase provides a definition for the list of selected experts and the preference of each expert within a single criterion. In this step, the EDM is constructed. The main parts of the EDM are the decision criteria and the alternatives, as shown in Table 14.

In Table 14, a crossover is identified between the criteria and the experts, and each of these experts determines each criterion importance level. 
Table 14 Criteria and alternatives

\begin{tabular}{lllll}
\hline Experts & Criteria & & & \\
\cline { 2 - 5 } & $\mathrm{C} 1$ & $\mathrm{C} 2$ & $\ldots$ & $\mathrm{Cn}$ \\
\hline E1 & $\operatorname{Imp}(\mathrm{E} 1 / \mathrm{C} 1)$ & $\operatorname{Imp}(\mathrm{E} 1 / \mathrm{C} 2)$ & & $\operatorname{Imp}(\mathrm{E} 1 / \mathrm{Cn})$ \\
E2 & $\operatorname{Imp}(\mathrm{E} 2 / \mathrm{C} 1)$ & $\operatorname{Imp}(\mathrm{E} 2 / \mathrm{C} 2)$ & & $\operatorname{Imp}(\mathrm{E} 2 / \mathrm{Cn})$ \\
E3 & $\operatorname{Imp}(\mathrm{E} 3 / \mathrm{C} 1)$ & $\operatorname{Imp}(\mathrm{E} 3 / \mathrm{C} 2)$ & & $\operatorname{Imp}(\mathrm{E} 3 / \mathrm{Cn})$ \\
$\ldots$ & $\ldots$ & $\ldots$ & $\ldots$ & $\ldots$ \\
En & $\operatorname{Imp}(\mathrm{En} / \mathrm{C} 1)$ & $\operatorname{Imp}(\mathrm{En} / \mathrm{C} 2)$ & & $\operatorname{Imp}(\mathrm{En} / \mathrm{Cn})$
\end{tabular}

\subsubsection{Phase 4: Application of fuzzy memberships}

The difference between all the versions of FWZIC is presented depending on the type of fuzzy used.

3.2.4.1 First version: based on triangular fuzzy numbers set This version was presented in the work of Mohammed et al. 2021b, where FWZIC was employed with triangular fuzzy numbers. The main formulas utilised were the following:

- Definition formula

$$
\mu A(x)=\left\{\begin{array}{l}
0 \text { if } x<a \\
\frac{x-a}{b-a} \text { if } a \leq x \leq b \\
\frac{c-x}{c-b} \text { if } b \leq x \leq x \\
0 \text { if } x>c
\end{array}, \text { where } a \leq b \leq c .\right.
$$

- Summation formula

$$
\tilde{x}+\tilde{y}=\left(a_{1}+a_{2}, b_{1}+b_{2}, c_{1}+c_{2}\right)
$$

- Subtraction formula

$$
\tilde{x}-\tilde{y}=\left(a_{1}-c_{2}, b_{1}-b_{2}, c_{1}-a_{2}\right)
$$

- Multiplication formula

$$
\tilde{x} \times \tilde{y} \cong\left(\mathrm{a}_{1} \mathrm{a}_{2}, \mathrm{~b}_{1} \mathrm{~b}_{2}, \mathrm{c}_{1} \mathrm{c}_{2}\right)
$$

- Division formula

$$
\tilde{x} / \tilde{y} \cong\left(\mathrm{a}_{1} / \mathrm{c}_{2}, \mathrm{~b}_{1} / \mathrm{b}_{2}, \mathrm{c}_{1} / \mathrm{a}_{2}\right) .
$$

- Division on crisp value formula

$$
\tilde{x} / \alpha=\left(a_{1} / \alpha, b_{1} / \alpha, c_{1} / \alpha\right)
$$

- Defuzzification formula 


$$
\frac{(a+b+c)}{3}
$$

3.2.4.2 Second version: based on interval type-2 trapezoidal-fuzzy set IT2Tr This version was presented in the work of Krishnan (2021), where FWZIC was extended with triangular fuzzy numbers. The main formulas utilised were the following:

- Definition formula

$$
\begin{gathered}
H^{L}(x)= \begin{cases}\frac{h_{A}^{L}\left(x-a_{1}^{L}\right)}{a_{2}^{L}-a_{1}^{L}} & a_{1}^{L}<x<a_{2}^{L}, \\
h_{A}^{L} & h_{A}^{L}\left(a_{4}^{L}-x\right) \\
\frac{h_{2}^{L} \leq x \leq a_{3}^{L},}{a_{4}^{L}-a_{3}^{L}} & a_{3}^{L}<x<a_{4}^{L}, \\
0 & \text { otherwise }\end{cases} \\
H^{U}(x)= \begin{cases}\frac{h_{A}^{U}\left(x-a_{1}^{U}\right)}{a_{2}^{U}-a_{1}^{U}} & a_{1}^{U}<x<a_{2}^{U}, \\
\frac{h_{A}^{U}\left(a_{4}^{U}-x\right)}{h_{A}^{U}\left(a_{2}^{U} \leq x \leq a_{3}^{U},\right.} & a_{3}^{U}<x<a_{4}^{U} \\
a_{4}^{U}-a_{3}^{U} & \text { otherwise }\end{cases}
\end{gathered}
$$

- Summation formula

$$
\begin{aligned}
\tilde{A} \oplus \tilde{B} & =\left(a_{1}^{T}+b_{i}^{T} ; \min \left(H_{1}\left(\tilde{A}^{T}\right), H_{1}\left(\tilde{B}^{T}\right)\right), \min \left(H_{2}\left(\tilde{A}^{T}\right), H_{2}\left(\tilde{B}^{T}\right)\right): T \in\{U, L\} 9\right. \\
i & =1,2,3,4)
\end{aligned}
$$

- Subtraction formula

$$
\begin{aligned}
\widetilde{\tilde{A}} \odot \widetilde{\vec{B}} & =\left(a_{1}^{T}-b_{5-15}^{T} ; \min \left(H_{1}\left(\tilde{A}^{T}\right), H_{1}\left(\tilde{B}^{T}\right)\right), \min \left(H_{2}\left(\tilde{A}^{T}\right), H_{2}\left(\tilde{B}^{T}\right)\right): T \in\{U, L\},\right. \\
i & =1,2,3,4)
\end{aligned}
$$

- Multiplication formula

$$
\begin{aligned}
& \widetilde{\tilde{A}} \otimes \widetilde{\vec{B}}=\left(X_{l}^{T} ; \min \left(H_{1}\left(\tilde{A}^{T}\right), H_{1}\left(\tilde{B}^{T}\right)\right), \min \left(H_{2}\left(\tilde{A}^{T}\right), H_{2}\left(\tilde{B}^{T}\right)\right): T \in U, L\right. \\
& i=1,2,3,4) \\
& \text { where } \\
& X_{i}^{T}= \begin{cases}\min \left(a_{i}^{T} b_{i}^{T}, a_{i}^{T} b_{5-i}^{T}, a_{5-i}^{T} b_{i}^{T}, a_{5-i}^{T} b_{5-i}^{T}\right) & \text { if } i=1,2 \\
\max \left(a_{i}^{T} b_{i}^{T}, a_{i}^{T} b_{5-i}^{T}, a_{5-i}^{T} b_{i}^{T}, a_{5-i}^{T} b_{5-i}^{T}\right) & \text { if } i=3,4\end{cases} \\
& \text { and } T \in\{U, L\}
\end{aligned}
$$

- Division formula 


$$
\begin{aligned}
& \tilde{A} \oslash \tilde{B}=\left(Y_{i}^{T} ; \min \left(H_{1}\left(\tilde{A}^{T}\right), H_{1}\left(\tilde{B}^{T}\right)\right), \min \left(H_{2}\left(\tilde{A}^{T}\right), H_{2}\left(\tilde{B}^{T}\right): T \in\{U, L\}\right.\right. \\
& i= 1,2,3,4, \\
& \text { where } \\
& Y_{i}^{T}= \begin{cases}\min \left(\frac{a_{i}^{T}}{b_{i}^{T}}, \frac{a_{i}^{T}}{b_{5-i}^{T}}, \frac{a_{5-i}^{T}}{b_{i}^{T}}, \frac{a_{5-i}^{T}}{b_{5-i}^{T}}\right) & \text { if } i=1,2 \\
\max \left(\frac{a_{i}^{T}}{b_{i}^{T}}, \frac{a_{i}^{T}}{b_{5-i}^{T}}, \frac{a_{5-i}^{T}}{b_{i}^{T}}, \frac{a_{5-i}^{T}}{b_{5-i}^{T}}\right) & \text { if } i=3,4\end{cases} \\
& b_{j}^{T} \neq 0, j=1,2,3,4 \text { and } T \in\{U, L\} .
\end{aligned}
$$

- Division on crisp value formula.

if $c \geq 0$ then

$$
\begin{aligned}
\tilde{A} / c= & \left(\tilde{A}^{L} / c, \tilde{A}^{U} / c\right) \\
= & {\left[\left(a_{1}^{L} / c, a_{2}^{L} / c, a_{3}^{L} / c, a_{4}^{L} / c ; \varpi_{1 A^{\prime}}^{L}, \varpi_{2 A}^{L}\right)\right.} \\
& \left.\left(a_{1}^{U} / c, a_{2}^{U} / c, a_{3}^{U} / c, a_{4}^{U} / c ; \varpi_{1 A}^{U}, \varpi_{2 A}^{U}\right)\right]
\end{aligned}
$$

if $c \leq 0$ then

$$
\begin{aligned}
\tilde{A} / c= & \left(\tilde{A}^{L} / c, \tilde{A}^{U} / c\right) \\
= & {\left[\left(a_{4}^{L} / c, a_{3}^{L} / c, a_{2}^{L} / c, a_{1}^{L} / c ; \varpi_{1 A^{\prime}}^{L}, \varpi_{2 A}^{L}\right)\right.} \\
& \left.\quad\left(a_{4}^{U} / c, a_{3}^{U} / c, a_{2}^{U} / c, a_{1}^{U} / c ; \varpi_{1 A}^{U}, \varpi_{2 A}^{U}\right)\right]
\end{aligned}
$$

- Defuzzification formula

$$
\operatorname{Def}(\tilde{\tilde{A}})=\frac{1}{2}\left(\sum_{T \in\{U, L\}} \frac{a_{1}^{T}+\left(1+H_{1}\left(\tilde{A}^{T}\right)\right) a_{2}^{T}+\left(1+H_{2}\left(\tilde{A}^{T}\right)\right) a_{3}^{T}+a_{4}^{T}}{4+H_{1}\left(\tilde{A}^{T}\right)+H_{2}\left(\tilde{A}^{T}\right)}\right)
$$

3.2.4.3 Third version: based on Pythagorean fuzzy set This version was presented in the work of Albahri (2021), where FWZIC was extended with Pythagorean fuzzy sets. The main formulas utilised were the following:

- Definition formula

$$
\begin{aligned}
& P=\left\{m,\left(\mu_{p}(m), v_{p}(m)\right) \mid m \in M\right\} \\
& 0<\left(\mu_{p}(m)\right)^{2}+\left(v_{p}(m)\right)^{2} \leq 1
\end{aligned}
$$

- Degree of hesitancy formula

$$
\pi_{p}(m)=\sqrt{1-\left(\mu_{p}(m)\right)^{2}+\left(v_{p}(m)\right)^{2}}
$$

- Summation and aggregation formula 


$$
\operatorname{PFAG}\left(\tilde{p}_{1}, \tilde{p}_{2}, \ldots, \tilde{p}_{n}\right)=\left(\sqrt{1-\prod_{j=1}^{n}\left(1-\left(\mu_{j}\right)^{2}\right)}=\prod_{j=1}^{n}\left(v_{j}\right)\right)
$$

- Division formula

$$
\begin{aligned}
& p\left(m_{i}\right) \div p\left(m_{j}\right)=\left(\frac{\mu_{p}\left(m_{i}\right)}{\mu_{P}\left(m_{j}\right)}, \sqrt{\frac{v_{P}^{2}\left(m_{i}\right)-v_{P}^{2}\left(m_{j}\right)}{1-v_{P}^{2}\left(m_{j}\right)}}\right) \\
& \text { if } v_{P}\left(m_{i}\right) \geq v_{P}\left(m_{j}\right), \mu_{P}\left(m_{i}\right) \leq \min \left\{\mu_{P}\left(m_{j}\right), \frac{\mu_{p}\left(m_{j}\right) \pi_{P}\left(m_{i}\right)}{\pi_{p}\left(m_{i}\right)}\right\}
\end{aligned}
$$

- Division on crisp value formula

$$
p / \lambda=\left(\sqrt{1-\left(1-\left(\mu_{p}\right)^{2}\right)^{\frac{1}{2}}},\left(v_{p}\right)^{\frac{1}{2}}\right), \lambda>0
$$

- Defuzzification formula

$$
\alpha=\left(\mu_{\alpha}, v_{\alpha}\right) \text { be PFN, } s(\alpha)=\mu_{\alpha}^{2}-v_{a}^{2}, h(\alpha)=\mu_{\alpha}^{2}+v_{a}^{2}
$$

3.2.4.4 Fourth version: based on T-spherical fuzzy set This version was presented in the work of Alsalem (2021), where FWZIC was extended with T-spherical fuzzy sets. The main formulas utilised were the following:

- Definition formula

$$
\begin{aligned}
& P=\left\{m,\left(\mu_{d}(m), \mathrm{v}_{d}(m), s_{d}(m)\right) \mid m \in M\right\}, \\
& 0<\left(\mu_{d}(m)\right)^{T}+\left(v_{d}(m)\right)^{T}+\left(s_{d}(m)\right)^{T} \leq 1,
\end{aligned}
$$

- Degree of hesitancy formula

$$
\pi_{m}(m)=\sqrt[T]{1-\left(\mu_{d}(m)\right)^{T}+\left(v_{d}(m)\right)^{T}+\left(s_{d}(m)\right)^{T}} .
$$

- Summation and aggregation formula

$$
\mathrm{T}-\operatorname{SAM}\left(\tilde{p}_{1}, \tilde{p}_{2}, \ldots, \tilde{p}_{n}\right)=\left\{\left[1-\prod_{i=1}^{n}\left(1-\mu_{\tilde{p}_{i}}^{2}\right)\right]^{1 / T}, \prod_{i=1}^{n} \nu_{\tilde{p}_{i}},\left[\prod_{i=1}^{n}\left(1-\mu_{\tilde{p}_{i}}^{2}\right)-\prod_{i=1}^{n}\left(1-\mu_{\tilde{p}_{i}}^{2}-s_{\tilde{p}_{i}}^{2}\right)\right]^{1 / T}\right\}
$$

- Division formula

$$
p_{1} \oslash p_{2}=\left(\left(\frac{\left(\mu_{\mathrm{p} 1}^{\mathrm{T}}\left(2-\mu_{\mathrm{p} 2}^{\mathrm{T}}\right)\right.}{1-\left(1-\mu_{\mathrm{p} 1}^{\mathrm{T}}\right) \times\left(1-\mu_{\mathrm{p} 2}^{\mathrm{T}}\right)}\right)^{\frac{1}{T}}, \frac{\left(\nu_{\mathrm{p} 1}^{T}-\nu_{\mathrm{p} 2}^{T}\right)^{\frac{1}{T}}}{\left(1-\nu_{\mathrm{p} 1}^{T} \times \nu_{\mathrm{p} 2}^{T}\right)^{\frac{1}{T}}}, \frac{\left(\mathrm{s}_{\mathrm{p} 1}^{T}-\mathrm{s}_{\mathrm{p} 2}^{T}\right)^{\frac{1}{T}}}{\left(1-s_{\mathrm{p} 1}^{T} \times s_{p 2}^{T}\right)^{\frac{1}{T}}}\right) \text {, if } \quad \frac{\mu_{p 2}^{\mathrm{T}}}{\mu_{\mathrm{p} 1}^{\mathrm{T}}} \geq \frac{1-\mathrm{s}_{\mathrm{p} 2}^{T}}{1-\mathrm{s}_{\mathrm{p} 1}^{T}} \frac{1+\mathrm{s}_{\mathrm{p} 1}^{T}}{1+\mathrm{s}_{\mathrm{p} 2}^{T}} \geq 1 \text {. }
$$

- Division on crisp value formula 


$$
\tilde{P} \oslash \lambda=\left\{\left(1-\left(1-\mu_{\tilde{P}}^{T}\right)^{1 / \lambda}\right)^{1 / T}, \nu_{\tilde{P}}^{1 / \lambda}, \mathrm{s}_{\tilde{P}}^{1 / \lambda}\right\}
$$

- Defuzzification formula

$$
\text { Score } p=\mu p T-s p T
$$

3.2.4.5 Fifth version: based on q-rung orthopair environment fuzzy set This version was presented in the work of Albahri et al. 2021c, where FWZIC was extended with q-Rung Orthopair fuzzy sets. The main formulas utilised were the following:

- Definition formula

$$
\begin{aligned}
& P=\left\{m,\left(\mu_{d}(m), \mathrm{v}_{d}(m)\right) \mid m \in M\right\} \\
& 0<\left(\mu_{d}(m)\right)^{q}+\left(v_{d}(m)\right)^{q} \leq 1, \quad \text { where } \quad \mathrm{q} \geq 1 .
\end{aligned}
$$

- Degree of hesitancy formula

$$
\pi_{m}(m)=\sqrt[q]{\left(\mu_{d}(m)\right)^{q}+\left(v_{d}(m)\right)^{q}-\left(\mu_{d}(m)\right)^{q} \cdot\left(v_{d}(m)\right)^{q}} .
$$

- Aggregation formula

$$
\mathrm{q}-\operatorname{ROFA}\left(\tilde{a}_{1}, \tilde{a}_{2}, \ldots, \tilde{a}_{n}\right)=\left(1-\prod_{k=1}^{n}\left(1-\mu_{k}^{q}\right)\right)^{\frac{1}{q}}, \prod_{k=1}^{n} v_{k} .
$$

- Division formula

$$
p_{1} \oslash p_{2}=\left(\frac{\mu_{1}}{\mu_{2}}, \sqrt{\frac{v_{1}^{q}-v_{2}^{q}}{1-v_{2}^{q}}}\right), \text { if } \mu_{1} \leq \min \left\{\mu_{2}, \frac{\mu_{2} \pi_{1}}{\pi_{2}}\right\}, v_{1} \geq v_{2}
$$

- Division on crisp value formula

$$
p / \lambda=\left(\sqrt[q]{1-\left(1-\left(\mu_{p}\right)^{q}\right)^{\frac{1}{\lambda}}},\left(v_{p}\right)^{\frac{1}{\lambda}}\right), \lambda>0 .
$$

- Defuzzification formula

$$
S_{k}=\mu_{\mathrm{k}}^{q}-\mathrm{v}_{\mathrm{k}}^{q}, \quad \text { where } \quad \mathrm{q} \geq 1 .
$$

\subsubsection{Phase 5: Computing the final weight}

The final weight is computed in three sub steps, where the utilised equations for related calculation follow the adopted fuzzy environment for the different FWZIC versions: 
1. The ratio of fuzzification data is computed using Eqs. (16) and (19) for TFN, Eqs. (23) and (26) for IT2Tr, Eqs. (31) and (32) for PFN, Eqs. (37) and (38) for T-SFN, and Eqs. (43) and (44) for q-Rung)

2. The average values are computed to find the final fuzzy values of the weight coefficients of the evaluation criteria $(\widetilde{w 1}, \widetilde{w 2}, \ldots, \widetilde{w n})^{T}$ using Eq. (20) for TFN, Eq. (27) for IT2Tr, Eq. (33) for PFN, Eq. (39) for T-SFN and Eq. (45) for q-Rung.

3. Defuzzification is conducted to find the final weight. Lastly, the defuzzification methods are implemented to find the crisp weight value using Eq. (21) for TFN, Eq. (28) for IT2Tr, Eq. (34) for PFN, Eq. (40) for T-SFN and Eq. (46) for q-Rung. Prior to calculating the final values of the weight coefficients, the weight of importance of each criterion should be assigned given the sum of the weights of all criteria for the rescaling purpose applied in this stage.

\subsection{Fuzzy decision by opinion score method}

The FDOSM is a superior method for prioritising the alternative ranks based on expert opinion of the decision information based on the ideal value of the related criterion. Prior to utilising the method, its steps must be understood along with its various versions online. Six versions of FDOSM were published between 2020 and 2021. This section presents the steps for FDOSM. All the similar steps were discussed between versions, and the differences are highlighted in the table. All the stages are similar except for the fuzzy opinion matrices that depends on the fuzzy type used alongside FDOSM, aggregation and defuzzification formulas.

\subsubsection{First stage: data transformation unit}

The decision matrix is transformed into an opinion matrix using the following steps:

3.3.1.1 Step 1: Decision matrix creation This step involves the selection of an ideal solution for each sub criteria used in a matrix. The people in charge of this task are the same experts who gave the weights using the previous weighting method FWZIC. The formula used in selecting the ideal solution is as follows:

$$
A^{*}=\left\{\left[\left(\max _{i} v_{i j} \mid j \in J\right),\left(\min _{i} v_{i j} \mid j \in J\right),\left(O p_{i j} \in I . J\right) \mid i=1.2 .3 \ldots \ldots m\right]\right\},
$$

3.3.1.2 Step 2: Reference comparison This step involves a reference comparison between the ideal solution selected from previous step and all the remaining values for each criterion using the following formula:

$$
O p_{\text {Lang }}=\left\{\left(\left(\tilde{v}_{i j} \otimes v_{i j} \mid j \in J\right) \cdot \mid i=1.2 .3 \ldots . m\right)\right\},
$$

The comparison is performed using a Likert Scale. The last output of this step includes transforming the opinion matrix into fuzzy opinion matrix using the following formula: 


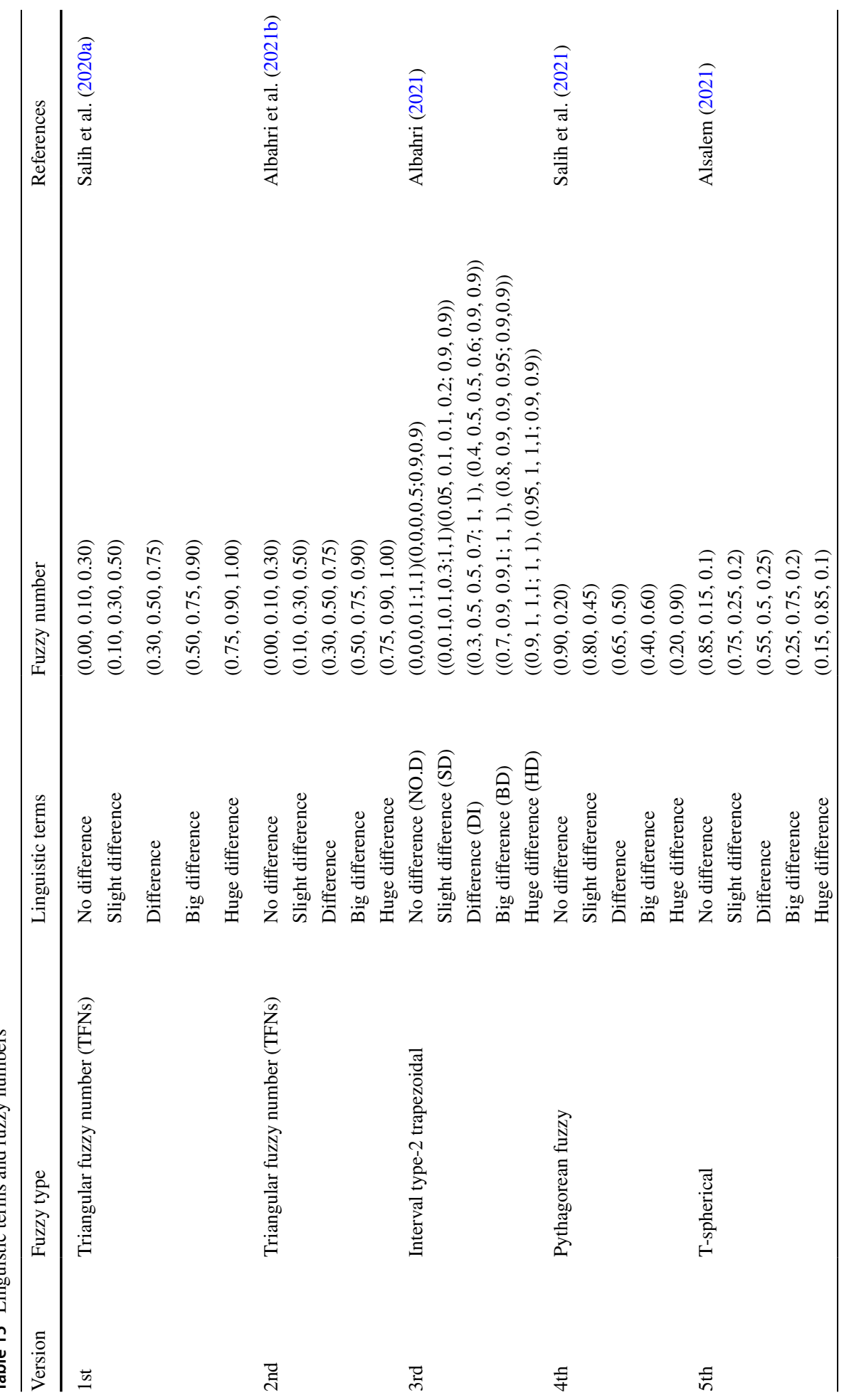




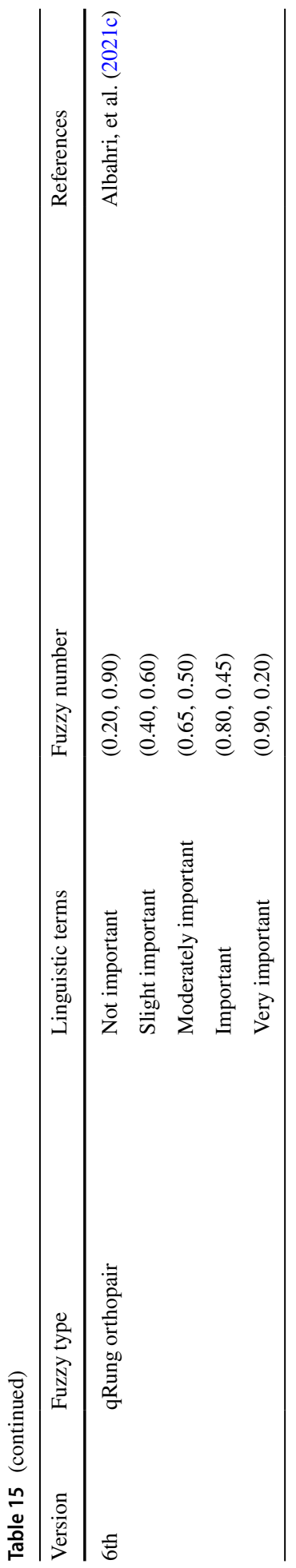




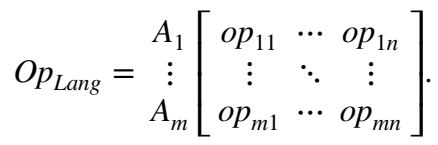

\subsubsection{Second stage: Data processing unit}

The opinion matrix that is the output from the previous stage is transformed into a fuzzy opinion matrix using the type of fuzzy extended with FDOSM. This conversion is done through converting the linguistic terms of the opinion matrix into fuzzy opinion matrix depending on the fuzzy type, as shown in the table. All six versions of FDOSM utilised different fuzzy opinion matrices. The first and second versions presented a triangular fuzzy number, as presented in Table 15.

After the transformation, the explicit criteria weights are fed into FDOSM. The opinion matrix from the previous step is aggregated into fuzzy using the aggregation equation that depends on the type of fuzzy used. Then, scoring is applied through transforming into crisp value. The aggregation and defuzzification equations of each version of the FDOSM as presented in Table 16.

Table 16 presents the related aggregation and scoring equations employed within each FDOSM version to find the final individual ranking results. Each version adapted one aggregation and one scoring (i.e. defuzzification) technique following the fuzzy environment. Except for the second version of FDOMS, different aggregation equations were implemented to compare the effect of changing operators on the final decision within the same fuzzy environment theoretically.

\section{Conclusion}

While the world continues to grapple with the effect of the COVID-19 pandemic, complementary efforts of various MCDM methods, evaluation and development categories are endeavouring to alleviate its effect of confused decision-making processes. Keeping that as the foundation of this work, we began this paper with a comprehensive review of the integration MCDM and COVID-19, in which we explored its taxonomy and classified the reviewed literature into evaluation-based and development-based for the first time. In the evaluation category, the studies discussed the assessment issue, which is related to decision-making problems from medical, social, economic, and technological aspects. It was found that the majority of these studies were medical, which revealed the MCDM requirement for evaluation and benchmarking for the health system in facing the unexpected medical crisis that COVID-19 caused. Meanwhile, the studies under the development category focused on providing new solutions in handling the decision-making issues related to COVID-19 either in service or patient-based. Their main efforts were on the service-based and vaccination phase.

We offered some of the latest characteristic points that matched using MCDM methods in the context of the COVID-19 pandemic and placed numerous implications to the readers. To calibrate the decision effect of the COVID-19 for different case studies, which is an urgent need, the bibliometric analysis discussed and presented the scientific productions, country, co-word, factor analysis and the collaboration around the world. The observed results show an increase in publication between 2020 and 2021, Asian countries 


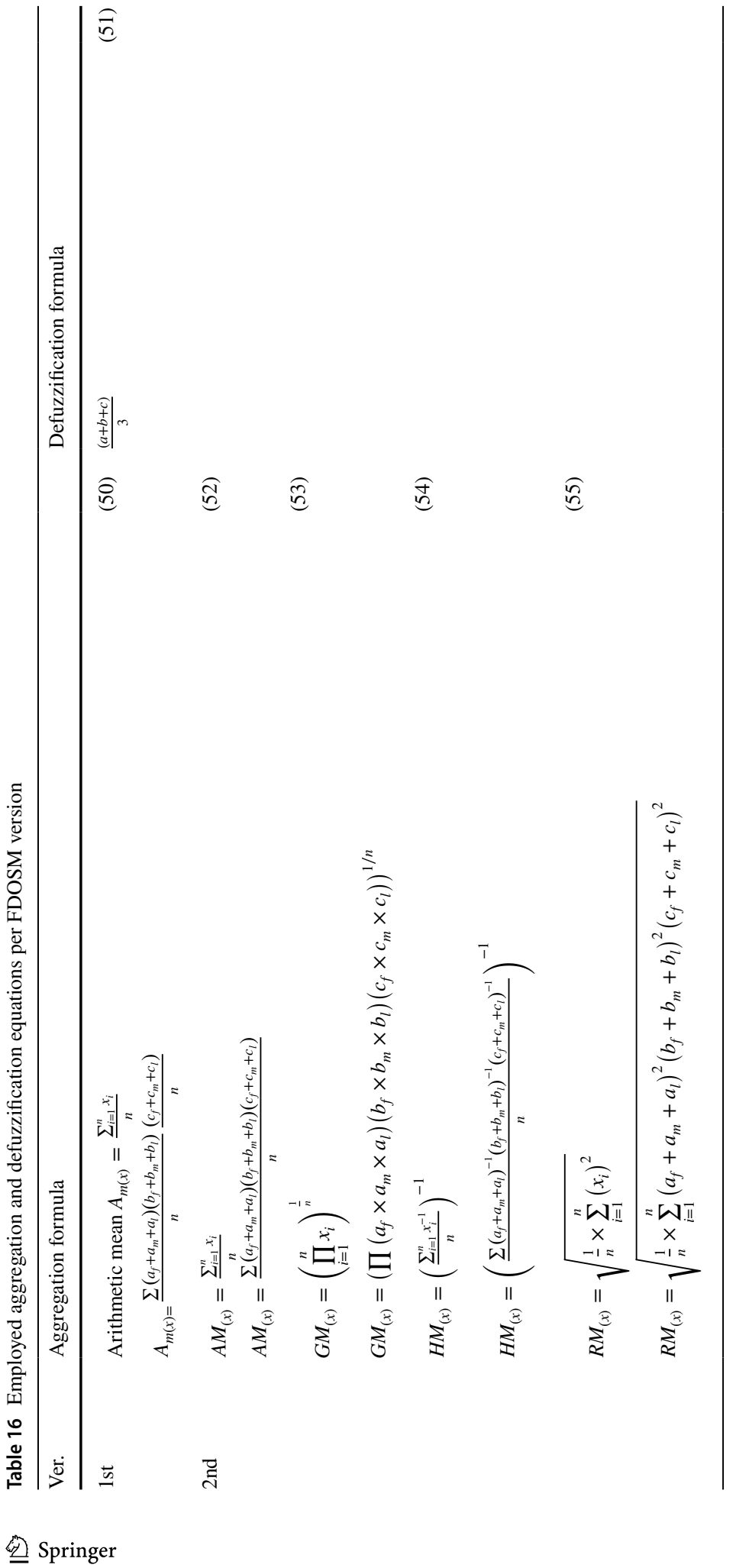




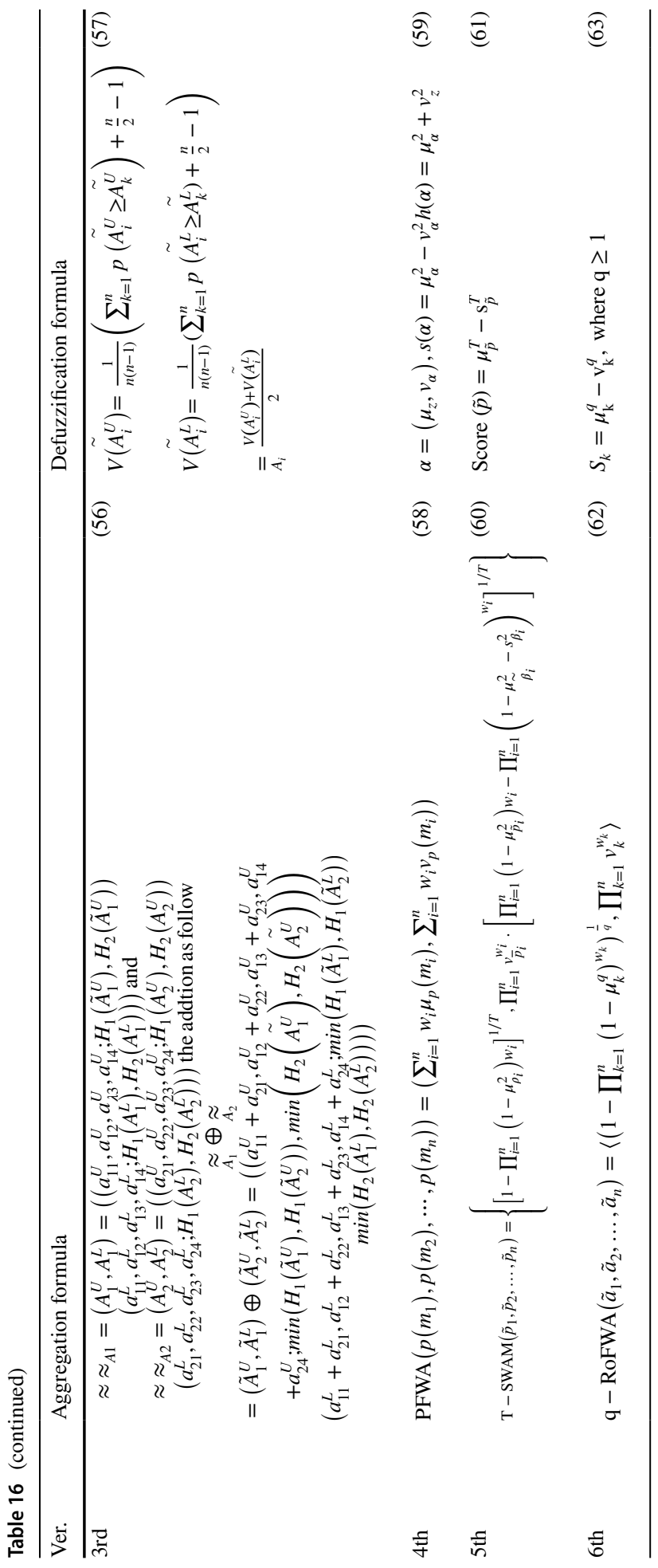


had the highest amount published, compared with Europe and the USA, wherein the scientific production covered widely interesting topics, but revealed the lack of collaboration in the world, which is mostly needed in facing the global effect of the COVID-19 pandemic. This study also analysed the determination of the criteria that are involved in the decision process of the COVID-19 context in terms of the followed method the criteria definition had set. It shows that the majority $(71.42 \%)$ of studies rely on criteria collected from the literature. The MCDM method with its two approaches (i.e., ranking and weightings) in the context of COVID-19 analyzed in its exiting, extension, and new versions within the adopted fuzzy environments and it's found that the majority of studies tended to utilize existing or extension MCDM method rather than the proposed new method in both categories (evaluation-based and development-based). Moreover, this study discussed the use of mathematical operators. It shows that all new and existing operators were applied for handling the decision information either for aggregation or distance measurement within the different fuzzy environments under the evaluation category, whilst some studies used the operator within the crisp numerical environment under the development category. The normalization technique in the MCDM method within the context of COVID-19 has been analysed. the results show that two normalization techniques are most commonly used in literature 12 different normalization techniques and that might belong to the low computation process compared to others. The decision-making context discussed and the individual and grouping aggregation process are indicated in this study in both categories, most of the studies employed the group aggregation internally, few of them used it externally or individually whilst some apply both for comparison purposes. With regard to the experts' selection studies, the based/conditions of expert/s selection are filtered and presented with the followed method. Noticeably, the base and the number of experts' selection in most of the study was without a specific guideline, which might affect the final decision that depends on expert consensus. Additionally, the validation schema under different parameters (i.e., computational complexity, number of alternative processes and criteria, adequacy in supporting group decision-making, addition or removal of criteria, different decision matrices) for the MCDM methods discussed found that only a few basic methods were examined. However, the other MCDM methods can employ this presented validation scheme and use it as a guideline for assessing the method appropriateness. The theoretical challenges of the MCDM are representing in unnatural comparison inconsistency, time consumption in a pairwise comparison, global weight (aggregating the weights), dynamic change, normalization, aggregation, external criteria weighting, reverse ranking and vagueness. Most of the studies in literature focused on handling the ambiguous and vagueness to improve the uncertainty, other theoretical challenges which not less important than vagueness and can affect the final decision, which received less attention.

Even though this study provided a distinctive and comprehensive analysis to the literature of the MCDM in the context of COVID-19 and highlighted the most recent characteristic points in the decision-making, this study is limited to the decision-making with regard to multicriteria methods, not including the systematic classification of MCDA methods throughout the article as follows: Ordinary/classical MCDA methods, Probabilistic/stochastic MCDA methods, Fuzzy MCDA models, Novel FMCDA models (hesitant, spherical, intuitionistic, etc., which could be included as a future suggestion to overcome this limitation.

Finally, we have taken a broad look at the future direction through a recommended methodology solution. In the thorough methodology theses, eight steps for FDM were presented: expert selection, developing expert form, dissemination and data collection, Likert scale conversion into fuzzy set, data analysis and threshold value, data analysis expert 
consensus \%, data analysis defuzzification process and fuzzy score value and data interpretation. For the second phase, FWZIC includes five stages as follows: criteria definition, SEJ, building the EDM, application of fuzzy memberships, and computing the final weight. For the third phase, FDOSM has been presented with two stages: data transformation unit and data processing unit. The responsibility to make the right decision for different fields in the context of COVID-19 rests largely with the presented MCDM methodology. Therefore, this type of study provides an outlook on future directions of such methodology which has the possibility to be applied for all COVID-19 cases reported in the literature. In these contexts and for these case studies, the presented methodology can identify problems, justify the used criteria, make a selection for the alternatives, implement the decision and evaluate the outcome.

Acknowledgements The authors are grateful to the Universiti Pendidikan Sultan Idris, Malaysia for funding this study under UPSI SIG Grant No. 2020-0150-109-01.

\section{References}

Abdel-Basst M, Mohamed R, Elhoseny MJHIJ (2020a) A model for the effective COVID-19 identification in uncertainty environment using primary symptoms and CT scans. Health Inform J 26(4):3088-3105

Abdel-Basst M, Mohamed R, Elhoseny M (2020) A model for the effective COVID-19 identification in uncertainty environment using primary symptoms and CT scans. Health Inform J 1460458220952918

Abdulkareem KH et al (2021) A new standardisation and selection framework for real-time image dehazing algorithms from multi-foggy scenes based on fuzzy Delphi and hybrid multi-criteria decision analysis methods. Neural Comput Appl 33:1029-1054

Abdulsalam Y, Hossain MS, and Engineering (2020) COVID-19 networking demand: an auction-based mechanism for automated selection of edge computing services. J Latex Class Files

Adler M, Ziglio E (1996) Gazing into the oracle: the Delphi method and its application to social policy and public health. Jessica Kingsley Publishers

Ahmad S, Mehfuz S, Beg J, Khan NA, Khan AH (2021) Fuzzy cloud based COVID-19 diagnosis assistant for identifying affected cases globally using MCDM. Mater Today Proc

Albahri $\mathrm{O}$ et al (2020) Helping doctors hasten COVID-19 treatment: Towards a rescue framework for the transfusion of best convalescent plasma to the most critical patients based on biological requirements via ml and novel MCDM methods. Comput Methods Programs Biomed 196:105617

Albahri A et al (2020) Multi-biological laboratory examination framework for the prioritization of patients with COVID-19 based on integrated AHP and group VIKOR methods. Int J Inf Technol Decis Mak 19(05):1247-1269

Albahri AS et al (2020b) Role of biological data mining and machine learning techniques in detecting and diagnosing the novel coronavirus (COVID-19): a systematic review. J Med Syst 44:1-11

Albahri O et al (2020d) Helping doctors hasten COVID-19 treatment: Towards a rescue framework for the transfusion of best convalescent plasma to the most critical patients based on biological requirements via ml and novel MCDM methods. Comput Methods Programs Biomed 196:105617

Albahri AS, Hamid RA, Albahri OS, Zaidan AJA (2021a) Detection-based prioritisation: Framework of multi-laboratory characteristics for asymptomatic COVID-19 carriers based on integrated EntropyTOPSIS methods. J Inform Sci 111:101983

Albahri OS et al (2021b) Multidimensional benchmarking of the active queue management methods of network congestion control based on extension of fuzzy decision by opinion score method. Int J Intell Syst 36(2):796-831

Albahri O et al (2021) Novel dynamic fuzzy decision-making framework for COVID-19 vaccine dose recipients. J Adv Res

Albahri A, Hamid RA (2020) Detection-based prioritisation: framework of multi-laboratory characteristics for asymptomatic COVID-19 carriers based on integrated entropy-TOPSIS methods. Artif Intell Med 101983

Albahri O et al (2020) Systematic review of artificial intelligence techniques in the detection and classification of COVID-19 medical images in terms of evaluation and benchmarking: taxonomy analysis, challenges, future solutions and methodological aspects. J Infect Public Health 13(10): 1381-1396. 
Albahri OS et al (2020a) Helping doctors hasten COVID-19 treatment: towards a rescue framework for the transfusion of best convalescent plasma to the most critical patients based on biological requirements via $\mathrm{ml}$ and novel MCDM methods 196:105617

Albahri $\mathrm{O}$ et al (2021a) Novel dynamic fuzzy decision-making framework for COVID-19 vaccine dose recipients

Albahri et al (2021) Integration of fuzzy-weighted zero-inconsistency and fuzzy decision by opinion score methods under a q-rung orthopair environment: a distribution case study of COVID-19 vaccine doses, p 103572

Albahri A et al (2021) Integration of fuzzy-weighted zero-inconsistency and fuzzy decision by opinion score methods under a q-rung orthopair environment: a distribution case study of COVID-19 vaccine doses. Comput Stand Interf 103572

Alsalem M et al (2021) Based on T-spherical fuzzy environment: a combination of FWZIC and FDOSM for prioritising COVID-19 vaccine dose recipients. J Infect Public Health

Alsalem OSAMA, Zaidan AA, Al-Obaidi JR, Mohammed RT, Alhamzah A, Alamoodi AH, Albahri AS, Zaidan BB, Jumaah FM (2021) Rescuing emergency cases of COVID-19 patients: an intelligent realtime MSC transfusion framework based on multicriteria decision-making methods

Althaf S, Babbitt CW (2021) Disruption risks to material supply chains in the electronics sector. Resour Conserv Recycl 167:105248

Altuntas F, Gok MS (2021) The effect of COVID-19 pandemic on domestic tourism: A DEMATEL method analysis on quarantine decisions. Int J Hosp Manage 92:102719

Aria M, Cuccurullo CJJ (2017) bibliometrix: An R-tool for comprehensive science mapping analysis. J Inform 11(4):959-975

Ashraf S, Abdullah S (2020) Emergency decision support modeling for COVID-19 based on spherical fuzzy information. Int J Intell Syst 35(11):1601-1645

Ashraf S, Abdullah S, Almagrabi AO (2020) A new emergency response of spherical intelligent fuzzy decision process to diagnose of COVID19. Soft Comput 1-17

Aytekin A (2021) Comparative analysis of the normalization techniques in the context of MCDM problems. Decis Making Appl Manage Eng 4(2):1-25

Baz A, Alhakami H, Alshareef E (2020) A framework of computational model for predicting the spread of COVID-19 pandemic in Saudi Arabia. Int J Intell Eng Syst

Bharsakade RS, Acharya P, Ganapathy L, Tiwari MK (2021) A lean approach to healthcare management using multi criteria decision making. OPSEARCH 1-26

Bodjanova S (2006) Median alpha-levels of a fuzzy number. Fuzzy Sets Syst 157(7):879-891

Boin A, 't Hart PJAJ (2010) Organising for effective emergency management: Lessons from research. Aust J Public Admin 69(4):357-371

Chang P-L, Hsu C-W, Chang P-C (2011) Fuzzy Delphi method for evaluating hydrogen production technologies. Int J Hydrogen Energy 36(21):14172-14179

Chaudhari S, More P, Bhadak S, Chaudhari S, Gawali T (2020) A survey on applications of artificial intelligence for enhancement in learning experience. AJCT 6(3):86-89

Chauhan A, Jakhar SK, Chauhan CJJ (2020) The interplay of circular economy with industry 4.0 enabled smart city drivers of healthcare waste disposal. J Clean Prod 279:123854

Cheng C-H, Lin Y (2002) Evaluating the best main battle tank using fuzzy decision theory with linguistic criteria evaluation. Eur J Oper Res 142(1):174-186

Chu H-C, Hwang G-J (2008) A Delphi-based approach to developing expert systems with the cooperation of multiple experts. Expert Syst Appl 34(4):2826-2840

Cousins, S. (2020). COVID-19 has "devastating" effect on women and girls. The Lancet 396(10247):301-302.

Dagens A et al (2020) Scope, quality, and inclusivity of clinical guidelines produced early in the covid-19 pandemic: rapid review. BMJ 369:m1936

Dawood KA, Sharif KY, Ghani AA, Zulzalil H, Zaidan A, Zaidan B (2021) Towards a unified criteria model for usability evaluation in the context of open source software based on a fuzzy Delphi method. Inform Softw Technol 130:106453

De Nardo P et al (2020) Multi-criteria decision analysis to prioritize hospital admission of patients affected by COVID-19 in low-resource settings with hospital-bed shortage. Int J Infect Dis 98:494-500

Demirjian NL et al (2020) Impacts of the coronavirus disease 2019 (COVID-19) pandemic on healthcare workers: a nationwide survey of United States radiologists. Clin Imag 68:218-225

Duan Y, Sun Y, Zhang Y, Fan X, Dong Q, Guo S (2021) Risk evaluation of electric power grid investment in china employing a hybrid novel MCDM Method. Mathematics 9(5):473

Duffield C (1993) The Delphi technique: a comparison of results obtained using two expert panels. Int J Nurs Stud 30(3):227-237 
Ecer F, Pamucar D (2021) MARCOS technique under intuitionistic fuzzy environment for determining the COVID-19 pandemic performance of insurance companies in terms of healthcare services. Appl Soft Comput 104:107199

Garai A (2013) Weighted intuitionistic fuzzy Delphi method. J Global Res Comput Sci 4(7):38-42

Garg H, Shahzadi G, Akram M (2020) Decision-making analysis based on Fermatean fuzzy Yager aggregation operators with application in COVID-19 testing facility. Math Probl Eng 2020

Ghaleb AM, Kaid H, Alsamhan A, Mian SH, Hidri L (2020) Assessment and comparison of various MCDM approaches in the selection of manufacturing process. Adv Mater Sci Eng 2020

Ghorui N et al (2021) Identification of dominant risk factor involved in spread of COVID-19 using hesitant fuzzy MCDM methodology. Res Phys 21:103811

Ghosh S, Das A, Hembram TK, Saha S, Pradhan B, Alamri AM (2020) Impact of COVID-19 induced lockdown on environmental quality in four indian megacities using landsat 8 OLI and TIRS-derived data and mamdani fuzzy logic modelling approach. Sustainability 12(13):5464

Glänzel W, Schubert A (2004) Analysing scientific networks through co-authorship. In: Handbook of quantitative science and technology research. Springer, pp 257-276

Gong J-W, Liu H-C, You X-Y, Yin L (2021) An integrated multi-criteria decision making approach with linguistic hesitant fuzzy sets for E-learning website evaluation and selection. Appl Soft Comput 102:107118

Grida M, Mohamed R, Zaied ANH (2020) Evaluate the impact of COVID-19 prevention policies on supply chain aspects under uncertainty. Transp Res Interdisc Persp 100240

Hamid RA, Albahri A, Albahri O, Zaidan AJJ, Computing H (2021) Dempster-Shafer theory for classification and hybridised models of multi-criteria decision analysis for prioritisation: a telemedicine framework for patients with heart diseases. J Ambient Intell Human Comput 33:1-35

He H, Harris LJJ (2020) The impact of Covid-19 pandemic on corporate social responsibility and marketing philosophy. J Bus Res 116:176-182

Hezam IM, Nayeem MK, Foul A, Alrasheedi AFJ (2021) COVID-19 vaccine: a neutrosophic MCDM approach for determining the priority groups. Res Phys 20:103654

Hezam IM, Khan Nayeem M, Foul A, Alrasheedi AF (2020) COVID-19 vaccine: a neutrosophic MCDM approach for determining the priority groups. Res Phys 103654

Ilyina LA, Tikhonov VS, Sakhno YS, Viktorova NV (2019) The mechanism of highly effective decision making in modern business systems. In: The leading practice of decision making in modern business systems. Emerald Publishing Limited

Io H, Lee C (2017) Chatbots and conversational agents: a bibliometric analysis. In: 2017 IEEE international conference on industrial engineering and engineering management (IEEM). IEEE, pp 215-219

Ishikawa A, Amagasa M, Shiga T, Tomizawa G, Tatsuta R, Mieno H (1993) The max-min Delphi method and fuzzy Delphi method via fuzzy integration. Fuzzy Sets Syst 55(3):241-253

Jain R, Rana KB, Meena ML (2021) An integrated multi-criteria decision-making approach for identifying the risk level of musculoskeletal disorders among handheld device users. Soft Comput 1-11

Jamshidiantehrani M, Ahmadzadeh A, Rahimisadr M, Abdolmohammadi MJ (2020) Identifying and prioritizing the factors affecting the agility of the supply chain of pharmaceutical company using multicriteria decision-making methods in COVID-19 pandemic. Syst Rev Pharm 11(7):371-381

Kamarulzaman N, Jomhari N, Raus NM, Yusoff MZM (2015) Applying the fuzzy delphi method to analyze the user requirement for user centred design process in order to create learning applications. Indian $\mathrm{J}$ Sci Technol 8(32):1-7

Khurana S, Haleem A, Luthra S, Huisingh D, Mannan B (2020) Now is the time to press the reset button: helping India's companies to become more resilient and effective in overcoming the impacts of COVID-19, climate changes and other crises. J Clean Prod 280:124466

Krishnan E et al (2021) Interval type 2 trapezoidal-fuzzy weighted with zero inconsistency combined with VIKOR for evaluating smart e-tourism applications. Int J Intell Syst

Lam WS, Lam WH, Jaaman SH, Liew KF (2021) Performance evaluation of construction companies using integrated entropy-Fuzzy VIKOR Model. Entropy 23(3):320

Mahanta J, Panda SJ (2020) A novel distance measure for intuitionistic fuzzy sets with diverse applications. Int J Intell Syst 2020

Manakandan SK, Rosnah I, Mohd RJ, Priya R (2017) Pesticide applicators questionnaire content validation: a fuzzy delphi method. Med J Malaysia 72(4):228-235

Manupati VK, Ramkumar M, Baba V, Agarwal A (2021) Selection of the best healthcare waste disposal techniques during and post COVID-19 pandemic era. J Cleaner Prod 281:125175

Mardani A, Saraji MK, Mishra AR, Rani P (2020) A novel extended approach under hesitant fuzzy sets to design a framework for assessing the key challenges of digital health interventions adoption during the COVID-19 outbreak. Appl Soft Comput J 96:106613 
Mardani A, Jusoh A, Nor K, Khalifah Z, Zakwan N, Valipour AJ (2015) Multiple criteria decision-making techniques and their applications - a review of the literature from 2000 to 2014. Econ Res Ekonomska Istraživanja 28(1):516-571

Mishra AR, Rani P, Krishankumar R, Ravichandran K, Kar S (2021) An extended fuzzy decision-making framework using hesitant fuzzy sets for the drug selection to treat the mild symptoms of Coronavirus Disease 2019 (COVID-19). Appl Soft Comput 103:107155

Mohammed TJ et al (2021) Convalescent-plasma-transfusion intelligent framework for rescuing COVID19 patients across centralised/decentralised telemedicine hospitals based on AHP-group TOPSIS and matching component. Appl Intell

Mohammed TJ et al (2021a) Convalescent-plasma-transfusion intelligent framework for rescuing COVID19 patients across centralised/decentralised telemedicine hospitals based on AHP-group TOPSIS and matching component. Appl Intell 51(5):2956-2987

Mohammed MA et al (2020) Benchmarking methodology for selection of optimal COVID-19 diagnostic model based on entropy and TOPSIS methods. IEEE Access

Mohammed RT et al (2021) Determining importance of many-objective optimisation competitive algorithms evaluation criteria based on a novel fuzzy-weighted zero-inconsistency method. Int $\mathbf{J}$ Inform Technol Decis Making 1-47

Mohammed R et al (2021) Determining importance of many-objective optimisation competitive algorithms evaluation criteria based on a novel fuzzy-weighted zero-inconsistency method. Int J Inform Technol Decis Making 1-47

Moreira DN, da Costa MP, and Psychiatry (2020) The impact of the Covid-19 pandemic in the precipitation of intimate partner violence 71:101606

Morovati Sharifabadi A, Naser Sadrabadi A, Dehghani Bezgabadi F, Peirow S (2016) Presenting a model for evaluation and selecting suppliers using interpretive structure modeling (ISM). Int J Indus Eng Prod Res 27(2):109-120

Moslem S, Campisi T, Szmelter-Jarosz A, Duleba S, Nahiduzzaman KM, Tesoriere GJS (2020) Bestworst method for modelling mobility choice after COVID-19: evidence from Italy. Sustainability 12(17):6824

Murray TJ, Pipino LL, Van Gigch JP (1985) A pilot study of fuzzy set modification of Delphi. Hum Syst Manag 5(1):76-80

Myers KR et al (2020) Unequal effects of the COVID-19 pandemic on scientists. Nat Human Behav 4(9):880-883

Naeem K, Riaz M, Peng X, Afzal D (2020). Pythagorean m-polar fuzzy topology with TOPSIS approach in exploring most effectual method for curing from COVID-19. Int J Biomath 2050075

Ocampo L, Yamagishi K (2020) Modeling the lockdown relaxation protocols of the Philippine government in response to the COVID-19 pandemic: an intuitionistic fuzzy DEMATEL analysis. Socio Econ Plan Sci 100911

Ortiz-Barrios M, Gul M, López-Meza P, Yucesan M, Navarro-Jiménez E (2020) Evaluation of hospital disaster preparedness by a multi-criteria decision making approach: the case of Turkish hospitals. Int J Disas Risk Reduc 49:101748

Pamučar D, Žižović M, Marinković D, Doljanica D, Jovanović SV, Brzaković P (2020) Development of a multi-criteria model for sustainable reorganization of a healthcare system in an emergency situation caused by the COVID-19 pandemic. Sustainability 12(18):7504

Pham TY, Ma HM, Yeo GT (2017) Application of fuzzy delphi TOPSIS to locate logistics centers in Vietnam: the logisticians' perspective. Asian J Shipp Logist 33(4):211-219

Powell C (2003) The Delphi technique: myths and realities. J Adv Nurs 41(4):376-382

Rahimianzarif E, Moradi M (2018) Designing integrated management criteria of creative ideation based on fuzzy delphi analytical hierarchy process. Int J Fuzzy Syst 20(3):877-900

Requia WJ, Kondo EK, Adams MD, Gold DR, Struchiner CJ (2020) Risk of the Brazilian health care system over 5572 municipalities to exceed health care capacity due to the 2019 novel coronavirus (COVID-19). Sci Total Environ 139144

Rod J, Oviedo-Trespalacios O, Cortes-Ramirez JJR (2020) A brief-review of the risk factors for covid-19 severity. Rev Saúde Pública 54:60

Rodríguez-Soler R, Uribe-Toril J, Valenciano JD (2020) Worldwide trends in the scientific production on rural depopulation, a bibliometric analysis using bibliometrix R-tool. Land Use Policy 97:104787

Salih MM, Zaidan B, Zaidan A (2020a) Fuzzy decision by opinion score method. Appl Soft Comput 96:106595

Salih MM, Zaidan B, Zaidan AJASC (2020b) Fuzzy decision by opinion score method. Phys Sens 96:106595

Salih MM, Albahri O, Zaidan A, Zaidan B, Jumaah F, Albahri A (2021) Benchmarking of AQM methods of network congestion control based on extension of interval type-2 trapezoidal fuzzy decision by opinion score method. Telecommun Syst 77(3):493-522 
Salman OH, Zaidan A, Zaidan B, Naserkalid F, Hashim MJIJ, Making D (2017) Novel methodology for triage and prioritizing using big data patients with chronic heart diseases through telemedicine environmental. Int J Inform Technol Decis Making 16(05):1211-1245

Samanlığlu F, Kaya BE (2020) Evaluation of the COVID-19 pandemic intervention strategies with hesitant F-AHP. J Healthcare Eng

Sarwar A, Imran M (2021) Prioritizing infection prevention and control activities for SARS-CoV-2 (COVID-19): a multi-criteria decision-analysis method. J Healthcare Leaders 13:77

Sayan M, Sarigul Yildirim F, Sanlidag T, Uzun B, Uzun Ozsahin D, Ozsahin I (2020) Capacity evaluation of diagnostic tests For COVID-19 using multicriteria decision-making techniques. Comput Math Methods Med 2020

Shah SAA, Longsheng C, Solangi YA, Ahmad M, Ali SJJ (2020) Energy trilemma based prioritization of waste-to-energy technologies: Implications for post-COVID-19 green economic recovery in Pakistan. J Clean Prod 124729

Sharma R, Shishodia A, Kamble S, Gunasekaran A, Belhadi AJ and Applications (2020) Agriculture supply chain risks and COVID-19: mitigation strategies and implications for the practitioners. Int J Logist Res Appl 1-27

Sheares GJ (2020) Internet of Things-enabled smart devices, biomedical big data, and real-time clinical monitoring in COVID-19 patient health prediction. Am J Med Res 7(2):64-70

Shirazi H, Kia R, Ghasemi P (2020) Ranking of hospitals in the case of COVID-19 outbreak: a new integrated approach using patient satisfaction criteria. Int J Healthcare Manage 1-13

Sultana I, Ahmed I, Azeem A (2015) An integrated approach for multiple criteria supplier selection combining Fuzzy Delphi, Fuzzy AHP \& Fuzzy TOPSIS. J Intell Fuzzy Syst 29(4):1273-1287

Thomas AJAP (2020) Plasma transfusion for acute covid-19 patients is willful ignorance of latent varicella zoster virus (VZV) reactivation forewarning threats

Vafaei N, Ribeiro RA, Camarinha-Matos LM (2018) Data normalisation techniques in decision making: case study with TOPSIS method. Int J Inform Decis Sci 10(1):19-38

Wan S-P, Chen Z-H, Dong J-Y (2021) An integrated interval type-2 fuzzy technique for democratic-autocratic multi-criteria decision making. Knowl Based Syst 214:106735

Ward MH et al (2018) Drinking water nitrate and human health: an updated review. IJERPH 15(7):1557

Wu J-Z, Tiao P-J (2018) A validation scheme for intelligent and effective multiple criteria decision-making. Appl Soft Comput 68:866-872

Wu L-C, Chang K-L, Liao S-K (2020) A hybrid MCDM model to select optimal hosts of variety shows in the social media era. Symmetry 12(1):125

Wu W, Xu Z (2020) Hybrid TODIM method with crisp number and probability linguistic term set for urban epidemic situation evaluation. Complexity 2020

Xu C, Luo X, Yu C, Cao S-J (2020) The 2019-nCoV epidemic control strategies and future challenges of building healthy smart cities. SAGE Publications, Sage

Yang Z, Li X, Garg H, Qi M (2020) decision support algorithm for selecting an antivirus mask over COVID19 pandemic under spherical normal fuzzy environment. Int J Environ Res Public Health 17(10):3407

Yang M-S, Ali Z, Mahmood T (2021) Complex q-rung orthopair uncertain linguistic partitioned bonferroni mean operators with application in antivirus mask selection. Symmetry 13(2):249

Yao S (2021) Fuzzy-based multi-criteria decision analysis of environmental regulation and green economic efficiency in a post-COVID-19 scenario: the case of China. Environ Sci Pollut Res 1-27

Zaidan AA (2021a) Based on T-spherical Fuzzy Environment: a combination of FWZIC and FDOSM for prioritizing COVID-19 vaccine dose recipients

Zaidan AA (2021b) Integration of fuzzy-weighted zero-inconsistency and fuzzy decision by opinion score method under a q-rung orthopair environment: a distribution case study of COVID-19 vaccine doses

Zamani-Sabzi H, King JP, Gard CC, Abudu S (2016) Statistical and analytical comparison of multi-criteria decision-making techniques under fuzzy environment. Oper Res Persp 3:92-117

Zeng Z, Chen P-J, Lew AAJTG (2020) From high-touch to high-tech: COVID-19 drives robotics adoption. Tour Geogr 22(3):724-734

Zhang X, Mao J, Lu Y (2020) A new computational method based on probabilistic linguistic Z-number with unbalanced semantics and its application to multi-criteria group decision making. IEEE Access

Zidan AA (2021) Novel dynamic fuzzy decision-making framework for COVID-19 vaccine dose recipients

Publisher's Note Springer Nature remains neutral with regard to jurisdictional claims in published maps and institutional affiliations. 


\section{Authors and Affiliations}

M. A. Alsalem ${ }^{1}$ - A. H. Alamoodi ${ }^{1}$ - O. S. Albahri ${ }^{1}$ D - K. A. Dawood ${ }^{2}$ - R. T. Mohammed ${ }^{2}$. Alhamzah Alnoor ${ }^{3}$ - A. A. Zaidan ${ }^{4}$ - A. S. Albahri ${ }^{5}$ B. B. Zaidan ${ }^{6}$ - F. M. Jumaah ${ }^{7,8}$. Jameel R. Al-Obaidi ${ }^{9}$

O. S. Albahri

osamah@fskik.upsi.edu.my

1 Department of Computing, Faculty of Arts, Computing and Creative Industry (FSKIK), Universiti Pendidikan Sultan Idris, Tanjung Malim, Malaysia

2 Department of Computing Science, Komar University of Science and Technology (KUST), Sulaymaniyah, Iraq

3 School of Management, Universiti Sains Malaysia, Pulau Pinang, Malaysia

4 Faculty of Engineering \& IT, British, University in Dubia, Dubai, United Arab Emirates

5 Informatics Institute for Postgraduate Studies (IIPS), Iraqi Commission for Computers and Informatics (ICCI), Baghdad, Iraq

6 Future Technology Research Center, National Yunlin University of Science and Technology, 123 University Road, Section 3, 64002 Douliou, Yunlin, Taiwan

7 Department of Advanced Applications and Embedded Systems, Intel Corporation, Plot 6 Bayan Lepas Technoplex, 11900 Pulau Pinang, Malaysia

8 Computer Engineering and Software Engineering Department, Polytechnique Montréal, Montréal, Canada

9 Department of Biology, Faculty of Science and Mathematics, Universiti Pendidikan Sultan Idris, 35900 Perak, Tanjong Malim, Malaysia 